\title{
Biomechanical analysis for abdominal aortic aneurysm risk stratification
}

Citation for published version (APA):

Speelman, L. (2009). Biomechanical analysis for abdominal aortic aneurysm risk stratification. [Doctoral Thesis, Maastricht University]. Datawyse / Universitaire Pers Maastricht. https://doi.org/10.26481/dis.20091210ls

Document status and date:

Published: 01/01/2009

DOI:

10.26481/dis.20091210ls

Document Version:

Publisher's PDF, also known as Version of record

\section{Please check the document version of this publication:}

- A submitted manuscript is the version of the article upon submission and before peer-review. There can be important differences between the submitted version and the official published version of record.

People interested in the research are advised to contact the author for the final version of the publication, or visit the DOI to the publisher's website.

- The final author version and the galley proof are versions of the publication after peer review.

- The final published version features the final layout of the paper including the volume, issue and page numbers.

Link to publication

\footnotetext{
General rights rights.

- You may freely distribute the URL identifying the publication in the public portal. please follow below link for the End User Agreement:

www.umlib.nl/taverne-license

Take down policy

If you believe that this document breaches copyright please contact us at:

repository@maastrichtuniversity.nl

providing details and we will investigate your claim.
}

Copyright and moral rights for the publications made accessible in the public portal are retained by the authors and/or other copyright owners and it is a condition of accessing publications that users recognise and abide by the legal requirements associated with these

- Users may download and print one copy of any publication from the public portal for the purpose of private study or research.

- You may not further distribute the material or use it for any profit-making activity or commercial gain

If the publication is distributed under the terms of Article $25 \mathrm{fa}$ of the Dutch Copyright Act, indicated by the "Taverne" license above, 


\section{Biomechanical Analysis for Abdominal Aortic Aneurysm Risk Stratification}


ISBN 978-90-9024718-2

(C) Lambert Speelman, Eindhoven 2009

Printed by Datawyse / Universitaire Pers Maastricht

Cover design by Jeroen den Doelder, Den Doelder Design 


\title{
Biomechanical Analysis for Abdominal Aortic Aneurysm Risk Stratification
}

\author{
PROEFSCHRIFT
}

ter verkrijging van de graad van doctor

aan de Universiteit Maastricht,

op gezag van de Rector Magnificus, prof. mr. G.P.M.F. Mols

volgens het besluit van het College van Decanen,

in het openbaar te verdedigen

op donderdag 10 December 2009 om 12.00 uur

door

Lambert Speelman

geboren op 1 augustus 1980 te Deventer 


\section{Promotores}

Prof. dr. M.J.H.M. Jacobs

Prof. dr. ir. F.N. van de Vosse (Technische Universiteit Eindhoven)

Copromotores

Dr.ir. E.M.H. Bosboom

Dr. G.W.H. Schurink

\section{Beoordelingscommissie}

Prof. dr. M.J. Post (voorzitter)

Prof. dr. F.P.T. Baaijens (Technische Universiteit Eindhoven)

Prof. dr. J.G. Maessen

Prof. dr. B.A.J.M. de Mol (VU Medisch Centrum Amsterdam)

Prof. dr. J.E. Wildberger

Financial support by the Netherlands Heart Foundation for the publication of this thesis is gratefully acknowledged.

Additional financial support was kindly provided by Stichting Hartsvrienden Rescar Maastricht, Philips Healthcare, Cook Medical, W.L. Gore \& Associates, Medtronic Bakken Research Center, and stichting onderzoek vaatchirurgie of the Catharina hospital Eindhoven. 


\section{Summary}

The risk of abdominal aortic aneurysm (AAA) rupture is nowadays mainly estimated based on the maximum diameter of the dilated aorta and, to a lesser extent, the growth rate of the diameter (Greenhalgh et al., 1998). However, from previous research it becomes clear that the maximum AAA diameter in some cases underestimates the risk of rupture, leading to unexpected ruptures, while in other cases the risk is overestimated (Darling et al., 1977; Lederle et al., 2002). In the latter case, the AAA may be unnecessarily repaired, with all accompanying surgical risks.

From a biomedical engineering perspective, rupture of an AAA occurs when locally the wall stress surpasses the strength of the wall. Wall stress may also have an effect on the growth rate of an AAA. High wall stress may lead to an increase in the amount of damage to the AAA wall, which may lead to remodeling and growth of the aneurysm. The objective of this thesis is to optimize and standardize AAA wall stress analyses for future diagnostic purposes by performing patient-specific wall stress analyses at different levels of complexity. Additionally, the relation between AAA wall stress, AAA growth rate, and biomarker concentrations is studied.

For the purpose of a future introduction of wall stress analyses as a clinical diagnostic tool, Philips Healthcare (Best, the Netherlands) developed a software package HemoDyn, in collaboration with the University Medical Center Utrecht (the Netherlands), Philips Research (Paris, France), Eindhoven University of Technology and our group (Breeuwer et al., 2008; de Putter et al., 2007; Delingette, 1994; Gérard et al., 2002; Ollabarriaga et al., 2005). This package is developed within the Philips ViewForum environment, which is widely used for visualization and analysis of medical images within clinical centers. HemoDyn is used for automatic segmentation and meshing of AAA's in the major part of the research in this thesis.

The first step in standardizing AAA wall stress analyses is to determine the reproducibility and robustness of the used system and the parameters. In chapter 2, reproducibility of the HemoDyn package was determined, by evaluating CTA data of 20 patients by three different operators. Besides the peak wall stress, also the 99-percentile wall stress was determined, computed as the highest wall stress in the AAA wall after exclusion of $1 \%$ of the wall with the highest stresses. The peak wall stress showed considerable variation within and between the operators. Clearly, small subtle shape changes induced by the different segmentations strongly influenced the peak wall stress. The 99-percentile wall stress is less sensitive to small geometrical variations and to the expertise and experience of the operator, and therefore a more 
robust and reproducible stress measure than the peak wall stress. A strong linear relation between the 99-percentile wall stress and the maximum AAA diameter was identified, although future research is needed to evaluate the accuracy of the proposed stress estimator as rupture risk predictor.

Besides reproducibility, also the effect of modeling assumptions on the wall stress needs to be evaluated. In chapter 3 , the effect of initial stress and nonlinear material behavior of the wall on the wall stress of patient-specific AAA models was studied. During CTA imaging, the AAA is subjected to the blood pressure and therefore contains an initial stress field. This initial stress can be determined prior to the stress computation and the results clearly showed that ignoring initial stress not only leads to a change in wall stress, but also that this change was different for each patient. Also the effect of material nonlinearity was different for each AAA. Therefore, no general correction factor could be used to estimate the effects on the wall stress and in future wall stress studies initial wall stress and material nonlinearity cannot be omitted.

The presence of intraluminal thrombus and calcifications in AAA's may have a strong influence on the resulting wall stress. In chapter 4 , the mechanical role of thrombus in wall stress analyses was evaluated by using idealized and patient-specific AAA models. Additionally, the growth rate of the AAA diameter was prospectively monitored and compared between AAA's with relative small and large thrombi. The results showed that a larger thrombus was associated with lower wall stress, but also with a higher AAA growth rate. This suggests that weakening of the AAA wall, under the influence of thrombus, may play a more imminent role in the process of AAA growth, than the AAA wall stress (vande Geest et al., 2006c; Vorp et al., 2001).

Chapter 5 discusses the effect of calcifications in the AAA wall on patient-specific wall stress. The effect of calcifications on the peak wall stress depends on the location, shape, and elastic modulus of the calcifications and cannot be estimated solely by the percentage of calcified wall. Although the location and shape of calcifications can easily be determined from the CTA data, the exact mechanical properties and the integration with the surrounding AAA wall need to be evaluated in future research. In chapter 6, a first step towards clinical application of AAA wall stress analyses has been made. In a relative small selection of AAA patients, wall stress has been studied in relation to biomarker concentrations in blood samples and AAA growth rate. It was hypothesized that AAA's with a relative high wall stress for the corresponding diameter, have increased damage and degeneration of the AAA wall. This might be reflected in increased AAA growth rate and up- or downregulation of specific circulating biomarkers. The AAA's with a relative higher wall stress appeared to have a higher growth rate. An increased growth rate was correlated to some of the biomarker concentrations. Although a trend could be identified between some concentrations and wall stress, none of the studied biomarker concentrations correlated significantly with wall stress. Further analysis is warranted to verify the relation between AAA wall stress, growth and biomarker concentrations.

Returning to the objective of this thesis, it can be concluded that a robust and reproducible stress measure is proposed in the form of the 99-percentile wall stress. The effect of initial stress and material nonlinearity was determined and it was 
concluded that they are indispensable in future wall stress analyses. Despite the fact that thrombus and calcifications have specific influence on the resulting wall stress, additional research is required to fully explore the mechanical effects of both factors. AAA wall stress was found to be related to AAA growth rate, but no correlation with biomarker concentrations could be identified at this moment. Hence, although the research in this thesis lays the foundation for AAA wall stress analyses to be incorporated in clinical diagnostics, future research is required to further optimize and evaluate AAA wall stress analyses for AAA growth and rupture risk prediction. 
viii Summary 


\section{Contents}

1 General Introduction 1

1.1 Abdominal Aortic Aneurysm . . . . . . . . . . . . . . . . 1

1.2 AAA Risk Stratification . . . . . . . . . . . . . . . . . 4

1.3 AAA Biomarkers . . . . . . . . . . . . . . . . . . . . 4

1.4 AAA Biomechanical Analysis . . . . . . . . . . . . . . . . 5

1.5 Objectives and Outline . . . . . . . . . . . . . . 7

2 AAA Wall Stress Analysis: 99-Percentile Versus Peak Stress 9

2.1 Introduction . . . . . . . . . . . . . . . . . . . . . . . . . . . . . . . . . .

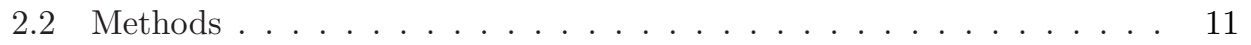

2.3 Results . . . . . . . . . . . . . . . . . . . . . . . . . . . . . . . . . . . . . . . . .

2.4 Discussion . . . . . . . . . . . . . . . . . . . . . . . . . . . . . . . . . . . . . . . . . .

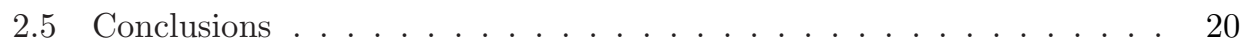

3 AAA Wall Stress Analysis: Initial Stress and Material Nonlinearity 21

3.1 Introduction . . . . . . . . . . . . . . . . . . . . . . . . . 22

3.2 Methods . . . . . . . . . . . . . . . . . . . . . 23

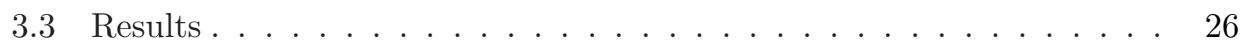

3.4 Discussion . . . . . . . . . . . . . . . . . . . . . . . . . . . . . . . . . . . . . . . 29

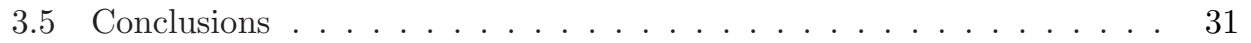

4 The Mechanical Role of Thrombus on the Growth Rate of an AAA 33

4.1 Introduction . . . . . . . . . . . . . . . . . . . . . . . 34

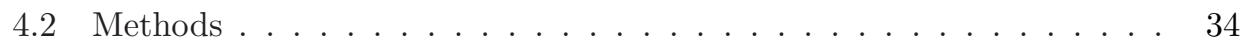

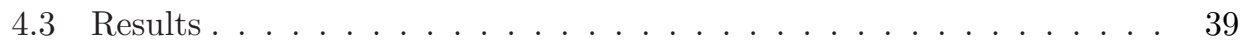

4.4 Discussion . . . . . . . . . . . . . . . . . . . . . . . . . . . . . . . . . . . . . .

4.5 Conclusions . . . . . . . . . . . . . . . . . . . . . . . . . . . . . . . . . . . . . . . . . . . . .

5 Effects of Calcifications in AAA Wall Stress Analyses 47

5.1 Introduction . . . . . . . . . . . . . . . . . . . . . . . . . . . . . . . . . .

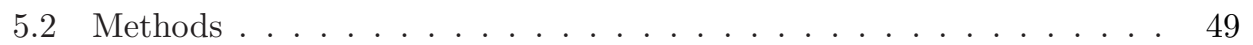

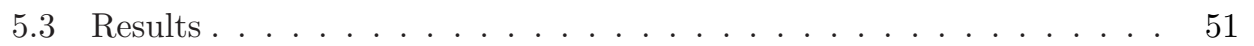

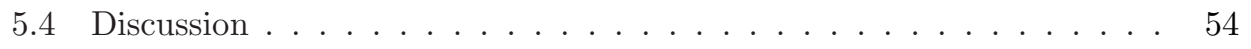

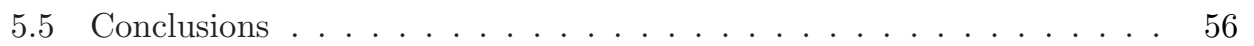


6 The Influence of Wall Stress on AAA Growth and Biomarkers 57

6.1 Introduction . . . . . . . . . . . . . . . . . 58

6.2 Methods . . . . . . . . . . . . . . . . . . . . . 59

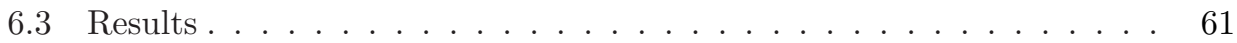

6.4 Discussion . . . . . . . . . . . . . . . . . . . 66

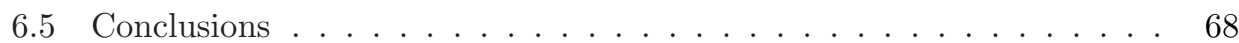

7 General Discussion $\quad 69$

7.1 Introduction . . . . . . . . . . . . . . . . . . . 70

7.2 Summary and Conclusions . . . . . . . . . . . . . . . . 71

7.3 Scope and Limitations ... . . . . . . . . . . . . . . . . . . 73

7.4 Clinical Perspectives . . . . . . . . . . . . . . . . . 75

7.5 General Conclusion . . . . . . . . . . . . . . . . . . . 75

A Finite Element Formulation of Mass and Momentum Balance Laws 77

References

89

$\begin{array}{ll}\text { Samenvatting } & 99\end{array}$

$\begin{array}{ll}\text { Dankwoord } & 103\end{array}$

$\begin{array}{ll}\text { Curriculum Vitae } & 105\end{array}$

$\begin{array}{ll}\text { List of Publications } & 107\end{array}$ 


\section{Chapter 1}

\section{General Introduction}

\subsection{Abdominal Aortic Aneurysm}

The aorta is the largest artery in the human body, transporting oxygenized blood directly from the left ventricle of the heart to the rest of the body. An aortic aneurysm is a local dilation in the aorta of more than 1.5 times the original diameter (Johnston et al., 1991). Although aneurysms can be present in every part of the aorta, the majority of the aortic aneurysms are situated in the abdominal aorta (AAA, figure 1.1), below the level of the renal arteries and above the aortic bifurcation to the
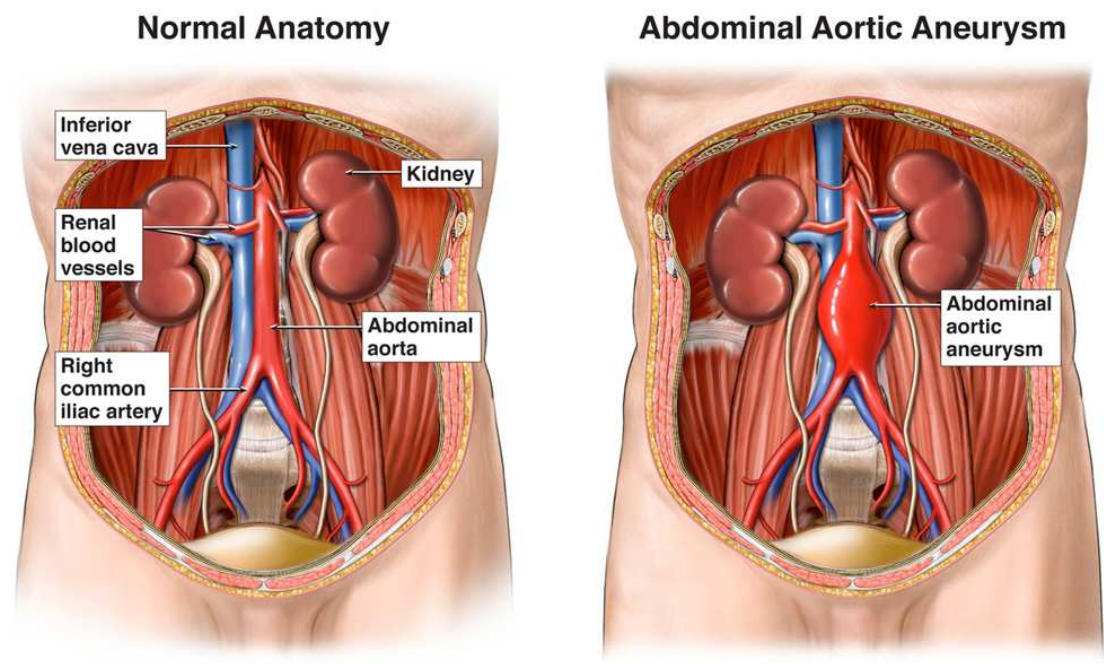

Medical Illustration Copyright $\mathcal{O} 2008$ Nucleus Medical Art. All Rights Reserved. www.nucleusinc.com

Figure 1.1: Normal aorta and abdominal aortic aneurysm (Medical Illustrations (C) 1999-2008 Nucleus Medical Art, Inc. All Rights Reserved. www.nucleusinc.com). 
common iliac arteries (Crawford and Cohen, 1982). A diameter of $3 \mathrm{~cm}$ or more is generally used as indication for an AAA. In most AAA's, thrombus is found between the perfused flow lumen and the aortic wall (figure 1.2). Thrombus is a fibrin structure with mainly blood cells, platelets and blood proteins, that is deposited onto the vessel wall (Harter et al., 1982). Also, calcification of the aortic wall is commonly seen in AAA patients (Harter et al. (1982), figure 1.2).

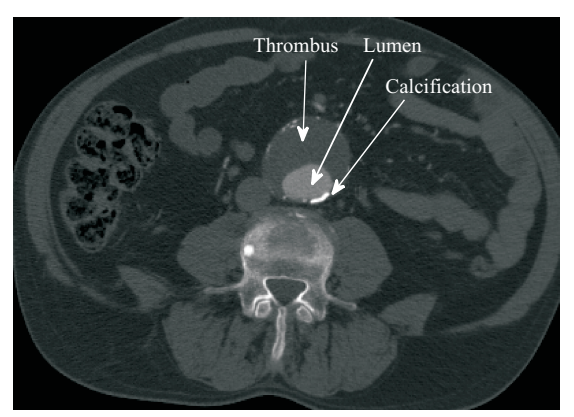

Figure 1.2: $C T A$ slice of an $A A A$.

AAA's occur mostly in the elderly population. In the Netherlands, the prevalence of AAA for people over 55 years of age is $4.1 \%$ in men and $0.7 \%$ in women (Pleumeekers et al., 1995). Risk factors for AAA include advanced age, male gender, smoking, hypertension, positive family history and atherosclerosis (Lederle et al., 1997; Pleumeekers et al., 1995).

AAA's are generally asymptomatic, until rupture of the AAA wall occurs. This can lead to a large abdominal bleeding and death within a short period of time. A ruptured AAA typically presents itself with acute abdominal pain and is in most cases combined with hemodynamic shock. The overall mortality rate for ruptured AAA's is around $90 \%$, as a large group of patients with a ruptured AAA does not reach the hospital in time (Basnyat et al., 1999). For those that reach the hospital in time, the mortality rates are as high as 50\% (Bown et al., 2002; Johnston, 1994). The overall mortality of AAA remains very high due to the fact that most AAA's are asymptomatic and therefore unknown. Screening programs for men between 65 and 75 years old, who have ever smoked, have been proposed previously (Kim et al., 2007; Thanos et al., 2008). Although screening can indeed reduce the AAA related mortality by $50 \%$, the costs per life-year remain considerable (Kim et al., 2007).

The main treatment option is to exclude the aneurysm wall from the systemic pressure, using a vascular graft. In open repair, a regular graft is sutured to healthy parts of the aorta by means of transabdominal surgery. This major surgical procedure has a 30-day mortality rate of about 5\% (Greenhalgh et al., 2004). Endovascular repair is an established alternative to open repair and is performed by placement of a stent-graft in the AAA, via a small incision in the groin. It is associated with a lower peri-operative mortality and a shorter recovery period than open repair 
(Greenhalgh et al., 2004). Also on the long term, aneurysm-related mortality is lower for endovascular repair (Lovegrove et al., 2008). However, complications like incomplete sealing, migration, kinking or material failure of the stent-graft may occur during the procedure or some time afterwards (Sandford et al., 2008). This leads to an elevated pressure inside the aneurysm and, in the worst case, rupture of the AAA wall (Wolters et al., 2007). Therefore, patients who underwent endovascular repair remain under surveillance, to evaluate the status of the stent-graft on a regular basis (Sandford et al., 2008).

\subsubsection{AAA Pathogenesis}

The aortic wall consists of three layers. The most inner layer is the tunica intima, consisting of a single layer of endothelial cells and a basal membrane. The tunica adventitia is the outer layer, containing connective tissue and the vasa vasorum (nurturing vessels for the arterial wall). The tunica media is the middle and thickest layer, consisting of smooth muscle cells with elastic fibers and collagen. A close collaboration between these three constituents is responsible for properties of the aortic wall. It is generally accepted that AAA is a degenerative disease of the tunica media (Daugherty and Cassis, 2002). The media of an AAA is characterized by a decrease in elastin (Campa et al., 1987) and smooth muscle cells (Lopez-Candales et al., 1997) and an increased infiltration of inflammatory cells (Shimizu et al., 2006), compared to a healthy aortic wall. Histology shows strong differences in the composition of healthy and aneurysm walls (Figure 1.3). These changes affect the integrity of the aortic wall, leading to a decrease in compliance and tensile strength and a progressive dilation of the wall.
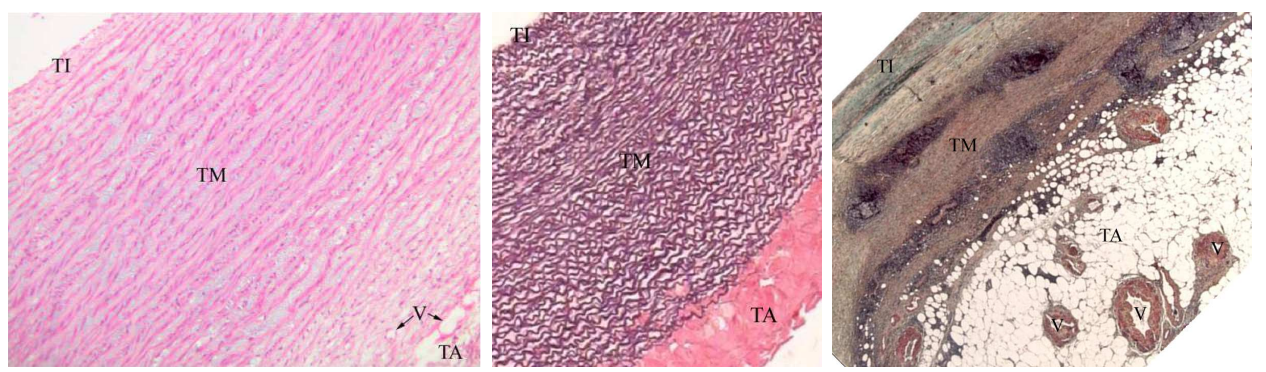

Figure 1.3: Histology of healthy (left and middle) and aneurysmatic (right) aortic wall. $T I$ is the tunica intima, TM the tunica media, TA the tunica adventitia and $V$ are bloodvessels of the vasa vasorum. The left image shows circumferentially oriented collagen fibers in the tunica intima. In the Elastin von Gieson staining (middle image), the elastin shows black and the collagen pink. The right image of the AAA wall shows a thickened tunica intima, loss of elastin, an increase in vasa vasorum and infiltration of fat cells in the tunica adventitia (images from www.medicalhistology.us (left and middle) and F.A. Hellenthal (right)). 


\subsection{AAA Risk Stratification}

To determine when an AAA requires surgical intervention, the risk of AAA rupture should carefully be weighed against the risk of the operative procedure. In current clinical practice, the risk of rupture is based on the maximum anterior-posterior diameter, and an elective repair procedure is initiated when the diameter reaches 5.5 $\mathrm{cm}$ or when the diameter grows more than $1 \mathrm{~cm}$ per year (Greenhalgh et al., 1998). Darling et al. (1977) studied 24,000 non-specific autopsy reports and found 473 nonresected AAA's, of which 118 were ruptured. Of the 265 aneurysms smaller than 5.0 $\mathrm{cm}, 12.8 \%$ were ruptured, indicating that for a significant number of small AAA's, the elective repair threshold of $5.5 \mathrm{~cm}$ in diameter is too high. Lederle et al. (2002) studied rupture rate of large AAA's with medical contraindication or patient refusal for elective repair. The 1-year incidence of probable rupture was $9 \%$ for AAA's with diameters $5.5-5.9 \mathrm{~cm}$. This increased to $33 \%$ for AAA's more than $7 \mathrm{~cm}$ in diameter. Although the 1-year incidence is substantially for AAA's over $5.5 \mathrm{~cm}$ in diameter, the majority of patients with large aneurysms will not (yet) experience rupture. These patients would be subjected to unnecessary surgical risks in case of elective AAA repair. For a more accurate rupture risk stratification, patient-specific parameters, other than the diameter, have to be considered.

Besides maximum diameter, it is advised to include the growth in diameter in the decision for AAA repair (Wolf et al., 1994). When a patient is known to have an AAA, follow-up is in most cases done by ultrasound examination to evaluate the maximum diameter and the diameter growth. The AAA growth is believed to increase with the initial diameter of the AAA (Stonebridge et al., 1996; Vega de Céniga et al., 2008). However, a large variation in growth rate is found between AAA's. While some AAA's remain stable for a considerable period of time, others show a strong increase in diameter in a short period or grow discontinuously, with alternating periods of growth and non-growth (Kurvers et al., 2004; Vega de Céniga et al., 2006). Prediction of the future expansion rate of an AAA in an early stage can be used to optimize the followup schedule and intervention plans for each patient.

So far, both aneurysm rupture and growth are unpredictable by the diameter alone. Better predictors for rupture and growth are required and may be found in a more extended patient-specific analysis, based on biomarker and biomechanical information. This may lead to an optimization of both the follow-up plan and the moment of aortic repair.

\subsection{AAA Biomarkers}

More insight in the pathogenic pathways of aneurysm formation and progression may be gathered by evaluating circulating biomarker concentrations (Golledge et al., 2008; Urbonavicius et al., 2008). Circulation matrix metalloproteinase-9 (MMP-9) concentrations have been investigated most frequently in association with AAA. MMP-9 is involved in the breakdown of the extracellular matrix and, in most studies, was found to be elevated in AAA's compared to healthy subjects (Golledge et al., 2008). Also, the tissue inhibitor of MMP 1 (TIMP-1) was found to be increased in 
AAA patients compared to healthy controls (Nakamura et al., 2000). The markers of inflammation interleukin-6 (IL-6) and C-reactive protein (CRP) are frequently studied in cardiovasculair disease and were found to be elevated in the presence of AAA in most studies (Golledge et al., 2008). Besides, serum CRP concentration was also associated with the size of AAA (Vainas et al., 2003).

Also, AAA growth was investigated in relation to biomarkers. Again, MMP-9 was correlated with AAA expansion rate (Lindholt et al., 2000). In the same study was found that alpha 1-antitrypsin $(\alpha 1-\mathrm{AT})$ was weakly correlated with AAA expansion rate (Lindholt et al., 2000). $\alpha 1$-AT is an inhibitor of alpha 1-trypsin, which inhibits elastase. Elastase, on its turn, actively breaks down elastic fibers in the aortic wall. Biomarkers as predictor for AAA rupture have been relatively little investigated. Engstrom et al. (2004) concluded that the incidence of fatal or repaired AAA was associated with a higher number and levels of inflammation-sensitive plasma proteins. CRP levels in patients with symptomatic or ruptured AAA's were significantly higher than in patients with an asymptomatic AAA (Domanovits et al., 2002). Also, MMP-1 and MMP-9 were elevated in the plasma of ruptured AAA versus non-ruptured AAA and elevation of MMP-9 was associated with ruptured AAA related 30-day mortality (Wilson et al., 2008).

Numerous studies have focused on measuring biomarker concentrations in order to predict AAA presence, growth or rupture, but most studies have not assessed the value of these markers as diagnostic tests for AAA (Golledge et al., 2008; Urbonavicius et al., 2008; Vainas et al., 2003; Vega de Céniga et al., 2009). Biomarkers may play a role in the identification of small AAA's. Additionally, when biomarkers associated with AAA are identified, targeted medical treatment may be developed to slow down AAA progression. For now, sensitivity and specificity appear inadequate for the use of single biomarkers alone in diagnosis (Golledge et al., 2008). Using multiple biomarkers in combination with other AAA related factors may in the future prove to be of value in the diagnostics of AAA.

\subsection{AAA Biomechanical Analysis}

From a (bio)medical engineering point of view, biomechanics play an imminent role in rupture of the AAA wall. When the stress on the AAA wall, caused by the blood pressure, locally exceeds the strength of the wall, rupture of the wall occurs. The law of Laplace states that the wall stress in a thin-walled cilinder linearly increases with increasing diameter and transmural pressure, and decreases for increasing wall thickness. However, due to the complex geometry of most AAA's, the wall stress is determined by the local AAA geometry and wall thickness, and can therefore not be predicted by the law of Laplace, or be based on simplified geometrical models (Hua and Mower, 2001).

Several research groups have previously focussed on wall stress as a clinical measure for rupture risk (Fillinger et al., 2002; Papaharilaou et al., 2007; Truijers et al., 2007; Venkatasubramaniam et al., 2004). Fillinger et al. (2002) found that peak wall stress for ruptured and symptomatic AAA's was significantly higher than for electively 
repaired AAA's. The same group concluded that peak wall stress was superior over maximum diameter for predicting AAA rupture (Fillinger et al., 2003). Truijers et al. (2007) found peak aortic wall stress at patient-specific blood pressure to be higher for ruptured AAA's than for asymptomatic AAA's.

The previously mentioned studies were based on patient group statistics, and a large overlap existed in wall stress of the ruptured and asymptomatic patient groups. Sensitivity and specificity is therefore still low for AAA wall stress as predictor for rupture risk.

Presently, most AAA wall stress studies are based on labor-intensive manual segmentation of the AAA's from the CTA data. This introduces a considerable amount of user variability. Heng et al. (2008) studied reproducibility and user variation of wall stress results, based on manual segmentation, and found that peak wall stress had only a moderate reproducibility and was susceptible to the experience and background of the user. To reduce the amount of user input, the AAA geometry should be segmented from CTA data in a more automatic fashion. For this purpose, Philips Healthcare (Best, the Netherlands) developed a software package HemoDyn, in collaboration with the University Medical Center Utrecht (the Netherlands), Eindhoven University of Technology, Philips Research (Paris, France) and our group (Breeuwer et al., 2008; de Putter et al., 2007; Delingette, 1994; Gérard et al., 2002; Ollabarriaga et al., 2005). This software is able to automatically segment AAA's from CTA data and to generate finite element meshes for wall stress computations. Hemodyn was used for AAA segmentation and meshing in the majority of the research presented in this thesis.

As in every model, assumptions and simplifications are made concerning factors that are difficult or impossible to measure. These factors include initial wall stress, intraluminal thrombus and aortic calcifications and it is not clear to what extent these simplifications influence the patient-specific wall stress. As AAA imaging is generally performed without cardiac triggering, the AAA is subjected to a time-averaged blood pressure and a certain amount of wall stress. Ignoring this initial stress not only leads to an overestimation of the AAA volume, but also to an underestimation of the wall curvature and the wall stress (de Putter et al., 2007). However, this initial stress may have different implications for the wall stress of different AAA's. Recently, material properties of thrombus were studied with compression and shear experiments, leading to far lower elastic moduli than previously assumed for thrombus (Ashton et al., 2009; Hinnen et al., 2007; van Dam et al., 2008). Re-evaluation of thrombus in wall stress analyses in the light of the recently obtained material properties may give more insight in the mechanical effect that thrombus has on patient-specific wall stress. The location of aortic calcifications can be identified from the CTA data (Siegel et al., 1994). Implementation of these calcifications in patient-specific AAA models can give information on the effect of calcifications on the resulting wall stress. 


\subsection{Objectives and Outline}

The objective of this thesis is:

to optimize and standardize AAA wall stress analyses for future diagnostic purposes by performing patient-specific wall stress analyses at different levels of complexity and to evaluate the relation between AAA wall stress, AAA growth rate, and biomarker concentrations.

Chapter 2 of this thesis evaluates the reproducibility and robustness of the wall stress as derived using an automated segmentation and meshing method using HemoDyn (Breeuwer et al., 2008). The 99-percentile wall stress is proposed as alternative stress measure for the peak wall stress. Chapter 3 discusses the effect of neglecting the initial stress on the patient-specific AAA wall stress. Additionally, the contribution of the nonlinear material behavior of the AAA wall is evaluated. Chapter 4 discusses the mechanical effect of intraluminal thrombus on the resulting wall stress for thirty patients. Also, idealized AAA models are used to estimate the effect on wall stress for a variety of thrombus stiffnesses and volumes. In chapter 5 , the influence of aortic wall calcifications on the AAA wall stress is studied, by comparing the results with and without calcifications for a number of AAA's, with varying amounts of calcifications. In chapter 6 , the relation between wall stress, AAA growth rate and circulating biomarker concentrations is studied for a group of patients. The hypothesis is posed that a higher wall stress leads to more damage and remodeling of the AAA wall, and therefore leads to an increase in AAA growth and a change in biomarker concentrations.

The concluding chapter of this thesis provides a summary of the results and a discussion of the previous chapters. 
8 Chapter 1 
Chapter 2

\section{Patient-Specific AAA Wall Stress Analysis: 99-Percentile Versus Peak Stress}

The objective of this study is to evaluate the robustness and reproducibility of AAA wall stress analysis using automated segmentation software. Additionally, patientspecific wall stress was studied in relation to the AAA diameter and other geometrical features.

The AAA's of twenty patients were segmented from CTA data by three operators with varying experience with the procedure and the interpretation of AAA CTA data. Both the peak stress and the 99-percentile stress were used for intra- and interoperator variability using the intraclass correlation coefficient (ICC). A regression analysis was performed to obtain a relation between the stress parameters and the maximum diameter. Outliers were analyzed by their geometrical characteristics.

The intra-operator ICC was 0.73 (op. 1) and 0.79 (op. 2) for the peak stress and 0.94 (op. 1 and 2) for the 99-percentile stress. The inter-operator ICC was 0.71 for the peak stress and 0.95 for the 99 -percentile stress. A linear relation between the 99-percentile stress and the diameter was significant. The tortuosity of the AAA's seemed to be larger for the AAA's with a relative high wall stress.

The 99-percentile wall stress is a more reproducible wall stress parameter than peak wall stress. Geometrical factors like AAA tortuosity should be evaluated more closely in relation to the AAA wall stress.

This chapter is based on: L. Speelman, E. M. Bosboom, G. W. Schurink, F. A. Hellenthal, J. Buth, M. Breeuwer, M. J. Jacobs and F. N. van de Vosse, Patientspecific AAA wall stress analysis: 99-percentile versus peak stress, Eur. J. Vasc. Endovasc. Surg. 2008, 36(6):668-676. 


\section{$2.1 \quad$ Introduction}

Reported incidence numbers of abdominal aortic aneurysms (AAA) are increasing due to general aging of the whole population and an increase in the amount of screening programs (Lederle et al., 1997; van der Vliet and Boll, 1997). If left untreated, an AAA will increase in size until rupture of the aortic wall occurs, causing a life-threatening hemorrhage. Repair procedures include open AAA surgery and endovascular repair using a stent-graft. Elective repair procedures have mortality rates between 1-4\% (Rutherford, 2006). For emergency repair in case of AAA rupture, mortality rates are as high as 50\% (Bown et al., 2002). The overall mortality rate for ruptured AAA's is around $90 \%$, as a large group of patients with a ruptured AAA does not reach the hospital in time (Basnyat et al., 1999; Johnston, 1994).

The current criterion for operative repair is an anterior-posterior diameter of the AAA of at least $5.5 \mathrm{~cm}$ (Greenhalgh et al., 1998). Statistically, the risk of rupture at this diameter is found to be equal to the risk of the repair procedure. In the past, several studies have concluded that the diameter may not be reliable as rupture risk criterion for the individual patient and that it should be replaced by a more patientspecific criterion (Darling et al., 1977; Nicholls et al., 1998; Vorp and vande Geest, 2005). Therefore, multiple studies have focused on patient-specific wall stress analyses (Fillinger et al., 2002; Raghavan et al., 2005; Venkatasubramaniam et al., 2004). Fillinger et al. (2002) studied the differences in peak wall stress between patients with elective and symptomatic or ruptured AAA's (mean AAA diameter 6.6 $\mathrm{cm}$ ) and found that peak wall stresses were significantly higher for the latter group. Truijers et al. (2007) computed peak wall stress in small AAA's and concluded that the wall stress at maximum systolic blood pressure is significantly higher for ruptured compared to asymptomatic aneurysms.

For the calculation of AAA wall stress, the geometrical shape of the AAA is reconstructed from medical images, in most cases Computed Tomographic Angiography (CTA). Accurate depiction of the AAA surface contour and shape by CTA reconstructive techniques requires a complex segmentation technique to reassemble adjacent axial slices, smooth surface transition between slices, maintain longitudinal orientation relative to the centerline, and accurately reproduce the relative tortuosity present in-vivo. Manual, user-generated segmentation has been shown to be time-consuming and leads to greater differences in derived wall stresses because of variability in AAA shapes created by different users (Fillinger et al., 2002; Venkatasubramaniam et al., 2004). Automation of segmentation may reduce shapeinduced stress variability due to the standardized smoothing and contouring steps in the procedure (Raghavan et al., 2005; Truijers et al., 2007).

Recently, the first study on inter- and intra-operator reliability of AAA wall stress analyses was published by Heng et al. (2008). Segmentation of the AAA's was performed by manual contour selection in each CTA slice of the AAA. Inter-operator variation was found over $100 \%$ between the lowest and highest stress value for the same patient. A double analysis on 10 patients by one operator resulted in differences up to $40 \%$. The small variations in geometry, introduced by the manual segmentation thus have a strong effect on the peak wall stress. 
The aim of this study is two-fold: 1) to evaluate the robustness and reproducibility of AAA wall stress analysis and 2) to relate wall stresses with the AAA diameter and other geometrical parameters. Since peak wall stress is found to be strongly dependent on local geometrical irregularities (Heng et al., 2008), also percentiles of stress are considered in the analysis. The 99-percentile stress is defined as the peak stress value in the AAA after exclusion of $1 \%$ of the total surface area with the highest stresses (de Putter et al., 2006a). This way, the absolute peak stress, possibly influenced by small geometrical variations, is excluded from the analysis. Intra- and inter-operator variations are determined for both the peak and 99-percentile wall stresses.

\subsection{Methods}

The contrast enhanced CTA scans of 20 patients were obtained from the University Medical Center Maastricht (the Netherlands, $n=11$ ) and the Catharina Hospital in Eindhoven (the Netherlands, $\mathrm{n}=9$ ). The CTA scans were performed in the arterial phase and the scans ranged from just distal to the descending thoracic aorta to the femoral artery bifurcation. A standard contrast enhanced hospital protocol for AAA's was used (in-plane resolution 512x512 pixels, field-of-view $30-35 \mathrm{~cm}^{2}$, reconstructed slice thickness 1-2 mm). Indirect brachial systolic, diastolic and mean arterial pressures (SP, DP and MAP) were recorded for all patients within 30 minutes after the CTA scan using a Dynamap 1846SX/P (Critikon Inc, Tampa, FL, USA). Information about the medical history and demographics for each patient was obtained. Research approval was given by the local Medical Ethics Commissions of the hospitals involved. All patients signed informed consent prior to the study.

\subsubsection{Segmentation and Mesh Creation}

The CTA data was processed using an automatic segmentation procedure that has been developed by Philips Healthcare (Best, the Netherlands) in collaboration with University Medical Center Utrecht, Eindhoven University of Technology, Philips Healthcare Research (Paris, France) and our group (Breeuwer et al., 2008; de Putter et al., 2007; Delingette, 1994; Ollabarriaga et al., 2005). The required user-input includes selecting a starting point proximal to the AAA and two end points, distal to the aortic bifurcation. Then, based on the Hounsfield values of the three user points, the centerline of the AAA was tracked automatically. Based on the centerline, a 3D active object (3DAO) was used to automatically detect the edges of the lumen and the AAA wall (figure 2.1). The 3DAO implementation is based on work by Delingette (1994) and has been previously used for segmentation of vascular structures, including AAA's (de Putter et al., 2005; Gérard et al., 2002; Ollabarriaga et al., 2005). Visual inspection of the 3DAO was done and manual corrections were made to the $3 \mathrm{D}$ surface by changing slice contours, where the user judges that the automatic segmentation is inaccurate (figure 2.2).

The segmentation of the AAA wall was used as input for the AAA wall stress simulations. The aortic bifurcation was excluded in case the iliac arteries were so strongly angulated, calcified or stenotic that segmentation was impossible. 


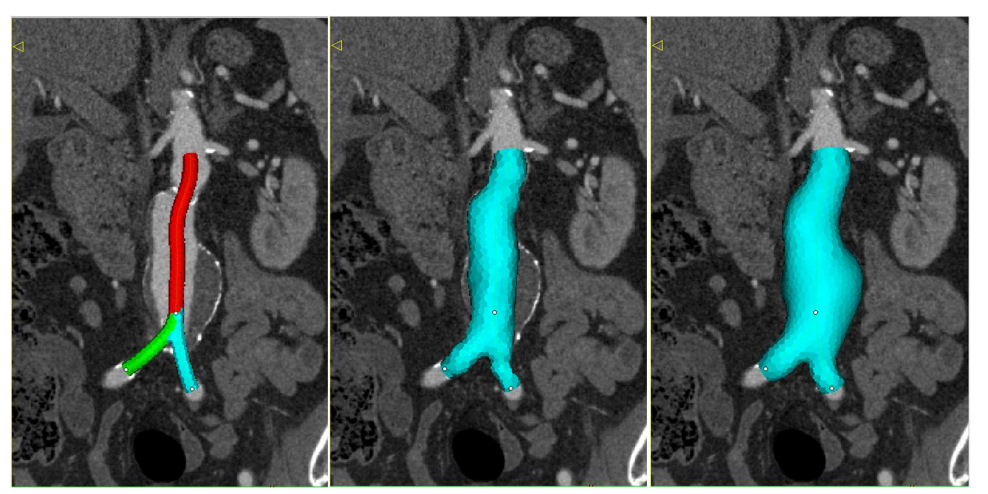

Figure 2.1: The $3 D A O$ of the centerline (left), the lumen (middle) and the $A A A$ wall (right).
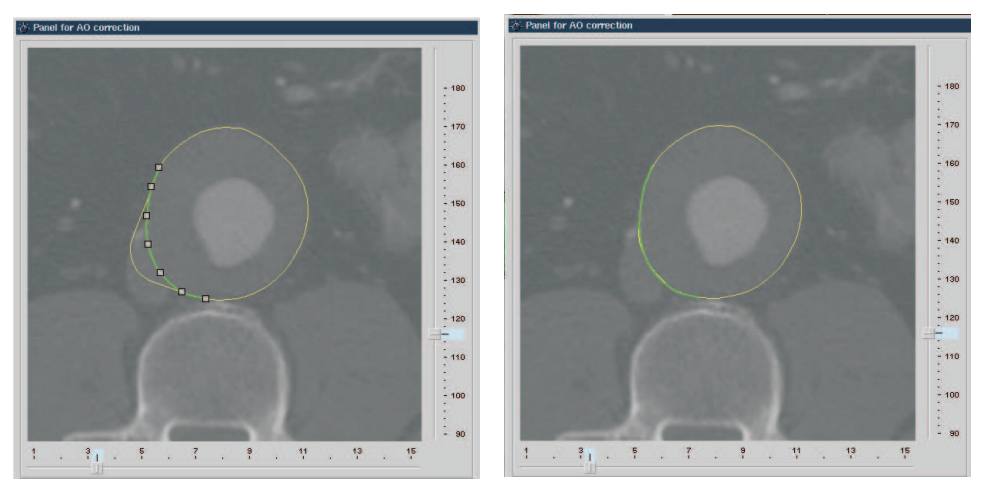

Figure 2.2: Failure of the automatic segmentation into the vena cava (thin solid line) and manual corrected contour (thick solid line, left). The corrected contour (right).

\subsubsection{AAA Wall Stress Analysis}

The commercially available finite element software Sepran (Sepra, Delft, the Netherlands) was used to calculate the AAA wall stresses, using the segmented AAA wall. A constant wall thickness of $2 \mathrm{~mm}$ was applied and an incompressible isotropic hyperelastic material model (shear modulus of $0.9 \mathrm{MPa}$ ) was used for the AAA wall. A mesh typically consisted of approximately 30,000 quadratic 15-node tetrahedral elements. A mesh refinement study was performed prior to the analyses and mesh independency was reached at this element-size. Tetrahedral elements were chosen as they could easily be automatically derived from the 3DAO segmentation and no mesh problems occur at the aortic bifurcation due to badly shaped elements. To enable comparison between patients, the patient group averaged systolic blood pressure is applied to the inner wall of the finite element model. The governing equations of mass and momentum were solved and the most distal and proximal planes are constrained 
in all directions as essential boundary conditions. Appendix A describes the finite element formulation of the mass and momentum balance laws.

Local stresses, strains and displacements were calculated. In each node, maximum principal stress is computed as a single stress measure. Besides the stress distribution, also the peak wall stress has been determined by computing the highest stress in the mesh. The 99-percentile stress is computed by first excluding $1 \%$ of all nodes containing the highest stress. Since all elements have the same size, this corresponds to $1 \%$ of the surface area of the wall. Thereafter, the highest stress of the remaining nodes is defined as the 99-percentile stress (de Putter et al., 2006a).

\subsubsection{Study Description}

Three operators assessed all 20 patients, of which two operators each performed the analysis 5 times per patient (operator 1 and 2). Operator 1 has in-depth knowledge about the segmentation and wall stress computation procedures and is experienced with interpreting CTA data from AAA patients, whereas operator 2 has no knowledge of the procedures and no experience with interpreting CTA data. All segmentations of the same AAA were made within a three week period and no blinding or scrambling of the CTA data was performed. Operator 3 is highly experienced with interpreting CTA data but has no knowledge about the computational procedures. On forehand, it was agreed to choose the starting point just distal to the ostia of the most proximal renal artery and the two end points a few centimeters distal to the aortic bifurcation, where curvature of the iliac arteries is minimum. Manual adaptation of the 3DAO was performed based on personal insight of each operator.

\subsubsection{Data Analysis}

Intra-operator variation was computed by the intraclass correlation coefficient (ICC). Complete agreement between observers is represented by an ICC of 1.0. To evaluate a possible learning effect, absolute difference in peak and 99-percentile stress between the first and second segmentation is compared to the difference between the last and second last segmentation. For the inter-operator variation, Bland-Altman plots were made and inter-operator ICC was computed. A simple regression analysis was performed to relate the peak stress and the 99-percentile stress with the maximum anterior-posterior diameter of the AAA, as determined by an experienced radiologist. The outliers in the regression analysis were compared with respect to their geometrical characteristics. The tortuosity of the AAA was proposed previously as risk parameter and therefore evaluated by determining the tortuosity of the central flow line and the central lumen line (Chaikof et al., 2002; Fillinger et al., 2004; Pappu et al., 2008). Here, the lumen is defined as the volume within the AAA wall, thus including intraluminal thrombus. The tortuosity of both the central lumen line (CLL) and the central flow line (CFL) is computed by dividing the distance along the central line between the lowest renal artery and the aortic bifurcation by the straight-line distance between these points (Chaikof et al., 2002). For the statistical analysis, Statgraphics Centurion XV (StatPoint, Herndon, Virginia, USA) was used. 


\subsection{Results}

The demographics of the patients are displayed in table 2.1. Compared to the demographics of other studies (Heng et al., 2008; Truijers et al., 2007), our patient group represents a typical sample selection from the population that suffers from an AAA.

Table 2.1: The demographical information of the patient group in this study.

\begin{tabular}{lc}
\hline \hline Demographics & Value \\
\hline Age(mean(range)) & $72(59-83)$ years \\
Gender (M:F) & $16: 4$ \\
Smoking & $15 \%$ \\
Drug controlled hypertension & $80 \%$ \\
Ischemic heart disease & $50 \%$ \\
COPD & $30 \%$ \\
Blood pressure (mean SP/DP/MAP) & $140 / 90 / 105 \mathrm{mmHg}$ \\
Maximum AP diameter (mean(range) & $49(44-57) \mathrm{mm}$ \\
\hline
\end{tabular}

\subsubsection{Reproducibility}

In the wall stress simulations, the patient group averaged $\mathrm{SP}$ of $140 \mathrm{mmHg}$ (18.7 $\mathrm{kPa}$ ) was used as input pressure. The time required to segment an AAA once was 10 minutes on average. None of the twenty AAA segmentations were completely rejected, although two AAA's were segmented without aortic bifurcation. Manual adaptation of slice contours were required for almost all AAA's to correct 3DAO ingrow in either the vena cava or other surrounding tissue. The computation of the wall stresses, strains and deformations took approximately 1 hour on average per geometry.

Figure 2.3 displays the wall stress distributions from the posterior side for one segmentation of two patients, as found by the three different operators. The location of the peak stress was identified to investigate if the peak stress is determined by the applied boundary conditions. Only a few AAA models showed a peak stress on one of the boundaries, resulting from the applied boundary conditions (11 of 220 models in total). The rest had the peak stress situated either at the aortic bifurcation (99 models) or at the beginning (44 AAA's) or end (66 AAA's) of the dilation.

Intra-operator variability. Operator 1 and 2 analyzed each patient 5 times. The mean and standard deviation of the peak and 99-percentile stress for all patients are given in figure 2.4 for both operators separately. The average standard deviation as a percentage of the mean for the peak stress is $11 \%$ (operator 1 , range $5-22 \%$ ) and $12 \%$ (operator 2, range $4-28 \%$ ), whereas for the 99 -percentile stress it is $2 \%$ (operator 1 , range $1-6 \%$ ) and $3 \%$ (operator 2 , range $1-7 \%$ ). The intra-operator intraclass correlation coefficient for operator 1 was 0.73 for peak stress and 0.95 for 99 -percentile stress. For operator 2 this was 0.79 and 0.94 respectively. 
The absolute differences between the first two and the last two segmentations were not significantly different for the peak and 99-percentile stress (p-value 0.19 and 0.43 for operator 1 and 0.33 and 0.84 for operator 2), indicating that no learning curve could be identified.

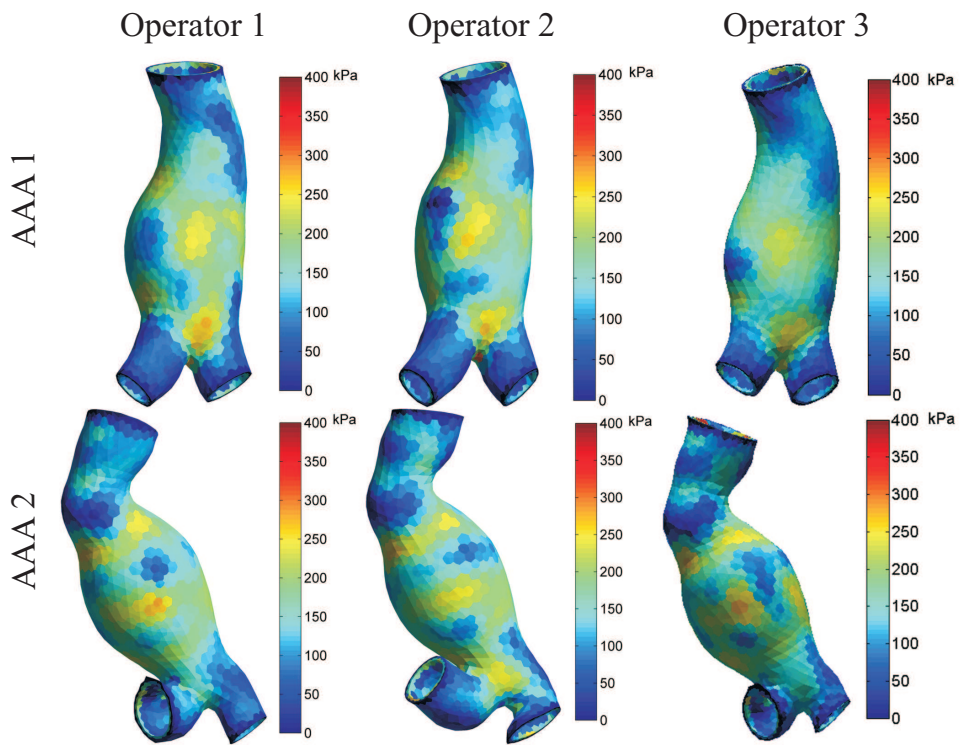

Figure 2.3: Posterior view of the stress distributions for two AAA's for operators 1, 2 , and 3 .
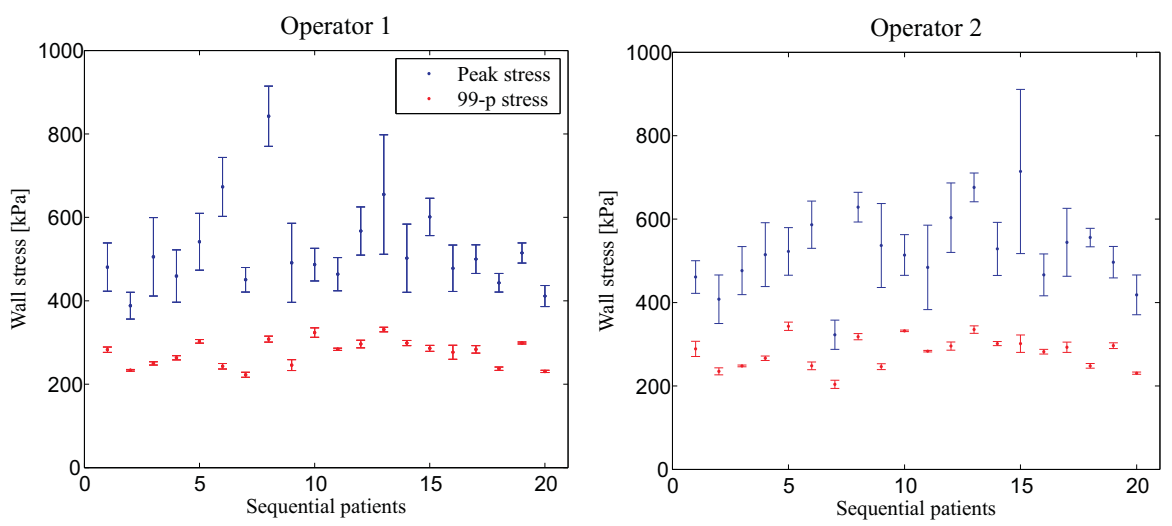

Figure 2.4: The mean and standard deviation of the peak stress and the 99-percentile stress for 5 analyses of all AAA's as determined by operator 1 (left) and 2 (right). 
Inter-operator variability. The number of manual adaptations were not tracked automatically, but all operators indicated that, on average, 2 to 5 adaptations were made in each AAA. The inter-operator intraclass correlation coefficient was 0.71 for the peak wall stress and 0.95 for 99-percentile stress. Figure 2.5 shows the Bland Altman plots for the peak stress and the 99-percentile stress, comparing all operators mutually. The mean differences $( \pm$ standard deviation $(\mathrm{SD}))$ between all operators are given in Table 2.2. From this it becomes clear that the average peak and 99percentile stress for operator 3 is lower than for operator 1 and on his turn lower than operator 2. The average differences are however small compared to the average stress values. The $\mathrm{SD}$ for the differences in peak stress is $76 \mathrm{kPa}$ on average, which is about $15 \%$ of the average peak wall stress. For the 99 -percentile stress, the average SD is $10 \mathrm{kPa}$, which is less than $4 \%$ of the average 99 -percentile stress.
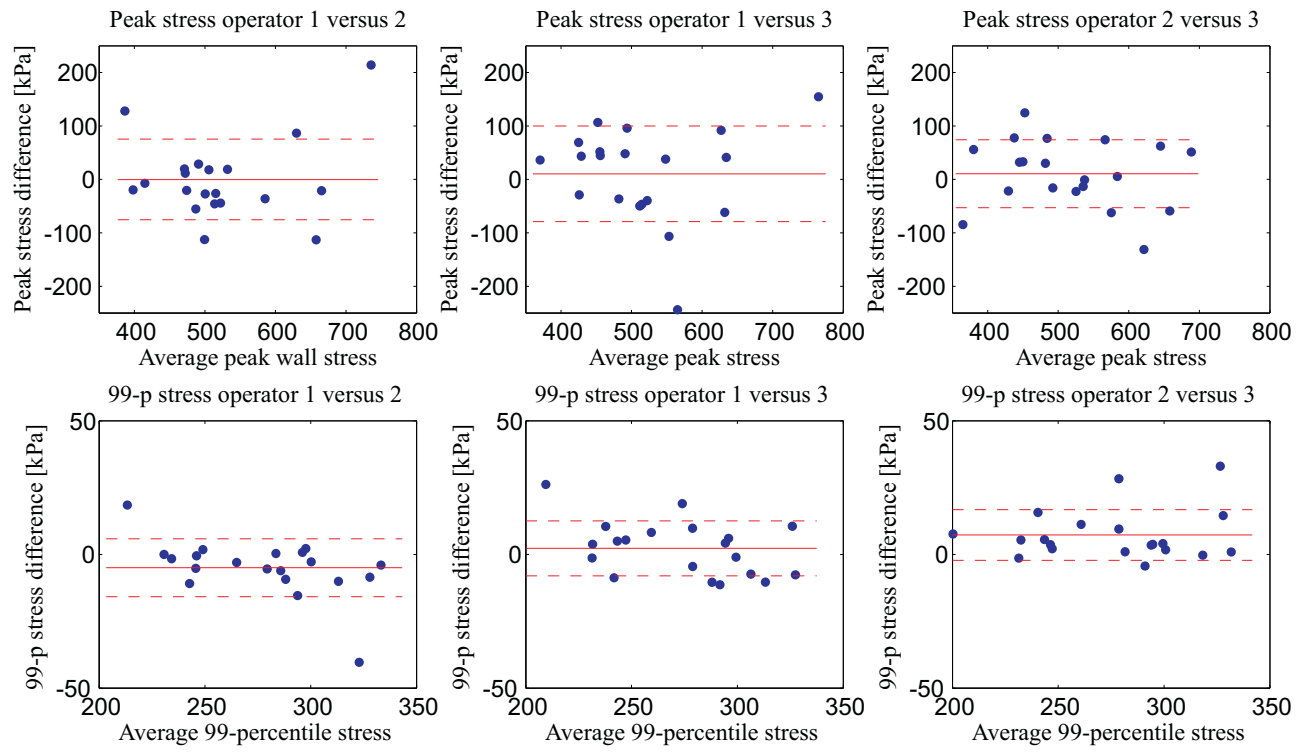

Figure 2.5: Bland Altman plots for operators 1, 2 and 3 (-- indicates one SD).

Table 2.2: Mean differences $( \pm S D)$ between all operators for peak and 99-percentile stress.

\begin{tabular}{lccc}
\hline \hline & Operator 1 - 2 & Operator 1 - 3 & Operator 2 - 3 \\
\hline Peak stress [kPa] & $-0.2 \pm 75.5$ & $10.4 \pm 89.4$ & $10.6 \pm 63.5$ \\
99-percentile stress [kPa] & $-5.0 \pm 10.8$ & $2.3 \pm 10.3$ & $7.3 \pm 9.5$ \\
\hline
\end{tabular}




\subsubsection{Patient Study}

To evaluate the relation between the AAA diameter and the stress estimates, first the stress estimates are averaged over all operators. A simple regression analysis did not lead to a significant relationship between the operator-averaged peak wall stress and the maximum diameter (p-value 0.19). Figure 2.6 shows the linear regression model between the operator-averaged 99-percentile stress and the maximum AAA diameter (left, $\mathrm{R}^{2} 0.75, \mathrm{p}$-value $<0.001$ ). The residuals between the computed stresses and the regression model are normalized by the SD of the residuals and plotted on the right in figure 2.6. It shows that five AAA's have stresses more than one SD above the predicted regression model (A-E). Four AAA's have values more than one SD beneath the model (F-I).
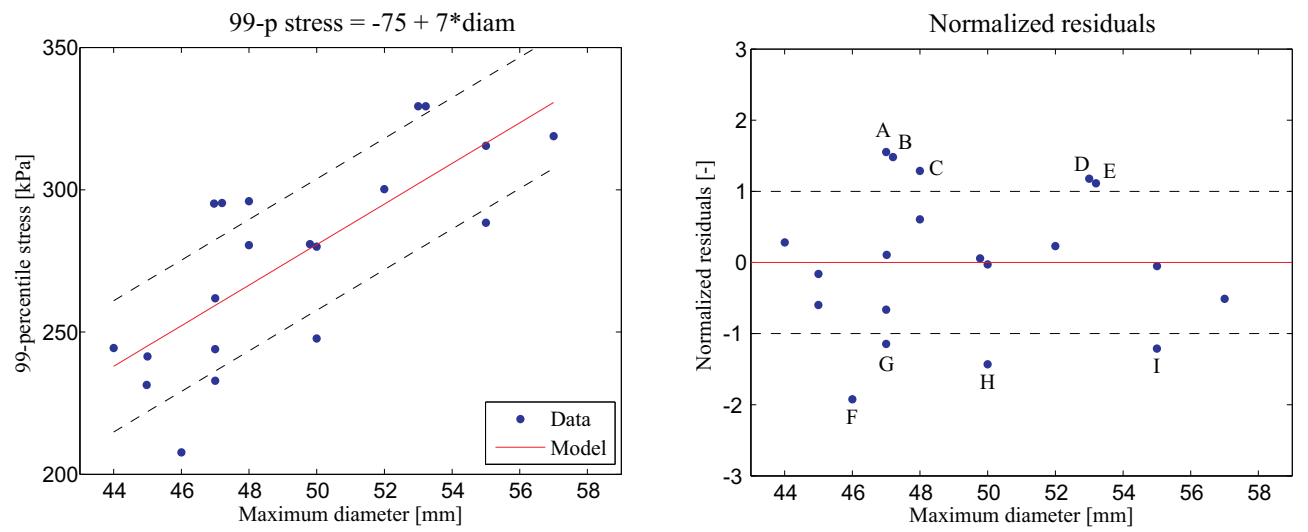

Figure 2.6: Linear regression between the 99-percentile stress and the maximum diameter (left, -- indicates one SD) and the residuals of the regression model, normalized to the standard deviation of the residuals (right).

Figure 2.7 A-I shows the geometries of the outliers. On visual inspection, the global geometries of the five high stress AAA's display a general tortuosity (figure 2.7 A$\mathrm{E}$ ). The four low stress AAA's appear to be less tortuous (figure $2.7 \mathrm{~F}-\mathrm{I}$ ). Table 2.3 displays the average tortuosity ( \pm SD) of the central lumen (CLL) and flow (CFL) line for the high, medium and low stress AAA's. Although not significant, the tortuosities of the CLL and CFL for the high stress AAA's is larger than for the medium stress AAA's and the low stress AAA's.

\subsection{Discussion}

Although the operators in this study all have different experience with the segmentation procedure and with reading CTA data of AAA's, they all were easily familiarized with the software and all found it easy and intuitively to use. This indicates that an easy transition into the clinic is possible once the software has proven to be of 


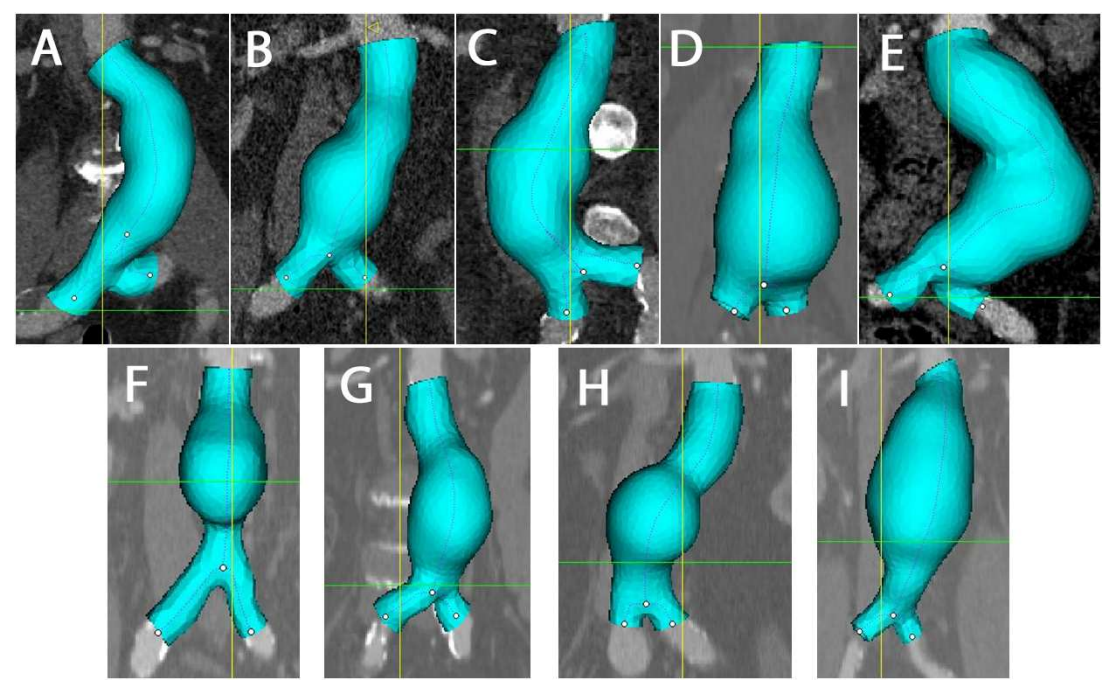

Figure 2.7: A-E High stress AAA's with stresses more than one standard deviation above the regression model. F-I Low stress AAA's with stresses more than one standard deviation below the regression model.

Table 2.3: Mean tortuosity ( $\pm S D$ ) of the $C L L$ and $C F L$ for high, medium and low stress AAA's.

\begin{tabular}{lcc}
\hline \hline & \multicolumn{2}{c}{ Tortuosity } \\
& Central lumen line & Central flow line \\
\hline High stress $(\mathrm{n}=5)$ & $1.66 \pm 0.49$ & $1.67 \pm 0.68$ \\
Medium stress $(\mathrm{n}=11)$ & $1.44 \pm 0.19$ & $1.21 \pm 0.21$ \\
low stress $(\mathrm{n}=4)$ & $1.33 \pm 0.09$ & $1.19 \pm 0.12$ \\
\hline
\end{tabular}

significant value in AAA diagnostics. Although not all AAA's could be segmented with the aortic bifurcation included, none were excluded from the study due to the inability to create a successful segmentation. The operators all indicated that all AAA's required 2 to 5 manual adaptations, which may explain the fairly high interoperator variability in the peak wall stress (figure 2.4).

From figure 2.3, it becomes clear that the stress distributions as derived from the segmentations of each operator show strong similarities. The location of the peak stress shows that most AAA's have the highest stress at a strongly curved part of the wall (either the bifurcation or the beginning or end of the dilation). Simply eliminating the stresses that arise at the boundaries due to the applied boundary conditions, thus does not improve the results. The location of the 99-percentile stress cannot be determined as this stress value may not be unique, but may occur on multiple locations on the AAA wall. 
Small subtle shape changes induced by the segmentation of different operators, however, appear to influence the magnitude of the stress estimates. The intra-operator ICC for the peak stress was 0.73 for operator 1 and 0.79 for operator 2 and 0.94 for the 99-percentile stress for both operators. Heng et al. (2008) found an intra-operator ICC for the peak stress of 0.84 , which is in the same range as our findings for the peak stress, but inferior to the correlation for the 99-percentile wall stress. No learning effect could be found for both operators as the differences between the first two and the last two segmentations were not significantly different. This indicates that the automatic software is insensitive to the experience and training of the user.

The inter-operator ICC for the peak stress was 0.71 which was identical to the findings by Heng et al. (2008). For the 99-percentile stress, an inter-operator ICC of 0.95 was found. The 99-percentile stress is therefore less sensitive to the type of user and their experience.

Subsequently, a regression model was fitted to both the peak stress and the 99percentile stress as a function of the diameter (figure 2.6). It may be expected that the stress relates to the diameter, as the diameter has proven to be a relatively good indicator of the rupture rate. Also, the law of Laplace states that an increased diameter leads to an increase in wall stress. No significant relation between peak wall stress and the maximum diameter could be found for the 20 AAA's in this study. For the 99-percentile stress, a linear relation was found with a moderate regression coefficient $\left(\mathrm{R}^{2} 0.75\right.$, p-value $\left.<0.001\right)$.

Nine outliers were identified outside one standard deviation of the regression model. The tortuosity of the CLL and CFL (table 2.3) is larger for the high stress AAA's although the standard deviation in this group was large. We observe that in addition to the maximum diameter, an increased aortic tortuosity might be associated with an increase in 99-percentile wall stress and thus rupture risk. This coincides with conclusions from a previous study (Pappu et al., 2008). Fillinger et al. (2004) studied anatomical characteristics for 259 ruptured and electively repaired AAA's. He found a greater risk for AAA's with no or mild tortuosity, when the groups were matched to an average diameter of $60 \mathrm{~mm}$. A larger patient population is required to further investigate this and other geometrical features that may influence the AAA wall stress. In this study, also lower percentiles of the stress were assessed. The inter-operator ICC of the 95-percentile stress was slightly better than for the 99-percentile stress (0.97 versus 0.95$)$, however, differences between patients were also smaller, leading to a lower discriminatory power. Future research may include a parameter study to find the percentile with the best ratio between intraclass correlation and discriminatory power.

This study has a number of limitations that may have influenced the results. Wall stress simulations in this study are all performed with a linear material model. Extending the current wall stress simulation with the nonlinear material model, that is proposed by Raghavan and Vorp (2000), may have an effect on the reproducibility results. This effect will however become apparent in both the peak and 99-percentile stress and we believe that this will not influence the conclusions of the present study. Future adaptations to our procedures will be made to compare the effect of a more complex material model for the AAA wall. 
Additionally, the choice of a constant wall thickness is a significant, but inevitable limitation. It is known that local wall thickness may strongly vary within AAA's, which may strongly influence the wall stresses (Thubrikar et al., 2001). However, non-invasive methods to measure local wall thickness are currently unavailable.

Initial stresses are caused by the pressure acting on the AAA during imaging, and are not accounted for in the wall stress simulations. Lu et al. (2008) studied the effect of initial stresses and concluded that the peak wall stress is overestimated, when initial stresses are not accounted for. This would again affect both the reproducibility of the peak and 99-percentile wall stress and therefore we believe that it does not influence the conclusions. Taking into account the initial stresses however does improve the accuracy of the model and incorporating initial stresses in our methods is currently under investigation.

No intraluminal thrombus was incorporated in the wall stress simulations as it was believed that the soft thrombus tissue (van Dam et al., 2008) propagates the intraaortic pressure in a fluid-like fashion, and hardly influences the wall stress results. This is supported by intra-thrombus pressure measurements by Schurink et al. (2000) on the AAA's of 9 patients during open repair. He found no reduction in mean and pulse pressure due to the presence of thrombus. Additionally, calcifications were not incorporated in the models, although we previously showed the significant effect that these calcium deposits may have on the wall stress (Speelman et al., 2007). The automatic procedures in this study, however, do not yet facilitate the determination and application of calcifications in the finite element models. Currently, our methods are extended with the option of incorporating thrombus and calcifications in the wall stress analysis.

\subsection{Conclusions}

The relative low intraclass correlations of the peak wall stress, found in this and previous research, limits the suitability of peak stress as reliable wall stress parameter. The 99-percentile stress proves to have a much higher reliability, by being insensitive to small geometrical variations and to the background and experience of the operator. Tortuosity of the AAA seems to be an important factor to identify high stress AAA's and may therefore be taken into account in the decision for an AAA repair procedure. A larger patient study with ruptured and non-ruptured AAA's is required to evaluate the accuracy of the proposed stress estimator. 
Chapter 3

\section{Initial Stress and Nonlinear Material Behavior in Patient-Specific AAA Wall Stress Analysis}

During CTA imaging of an AAA, the aneurysm is subjected to a time-averaged blood pressure and is therefore not stress free. The objective of this study is to evaluate the effect of neglecting this initial stress (IS) on the patient-specific AAA wall stress. Additionally, the contribution of the nonlinear material behavior of the AAA wall is evaluated.

Thirty patients with maximum AAA diameters below the current surgery criterion were scanned with contrast-enhanced CTA and the AAA's were segmented from the image data. The mean arterial blood pressure (MAP) was measured immediately after the CTA scan and used to compute the IS that corresponds with the CTA geometry. Wall stress was obtained with and without IS and with linear and nonlinear material properties and mutually compared.

Omitting initial stress and material nonlinearity in AAA wall stress computations leads to different effects in the resulting wall stress for each AAA. Therefore, provided that other assumptions made are not predominant, initial stress and a nonlinear material model should be used in future patient-specific AAA wall stress analyses.

This chapter is based on: L. Speelman, E. M. Bosboom, G. W. Schurink, J. Buth, M. Breeuwer, M. J. Jacobs and F. N. van de Vosse, Initial Stress and Nonlinear Material Behavior in Patient-Specific AAA Wall Stress Analysis, J. Biomech. 2009, 42(11):1713-1719. 


\subsection{Introduction}

An abdominal aortic aneurysm (AAA) is a local dilation in the abdominal aorta of more than $50 \%$ the original diameter and occurs in about 1 of 20 men over 65 years of age (Pleumeekers et al., 1995; van der Vliet and Boll, 1997). The largest threat for an AAA is rupture, which is fatal in more than $70 \%$ of the cases (Basnyat et al., 1999; Budd et al., 1989). Aortic repair is considered when the risk of rupture exceeds the risk of the surgical procedure. Currently, clinicians use the maximum AAA diameter as rupture risk estimator. A higher sensitivity and specificity was reached by computing the maximum AAA wall stress with finite element analysis, incorporating the total AAA geometry (Fillinger et al., 2002; Truijers et al., 2007). Clearly, extending the AAA risk estimation model with more patient-specific parameters thus leads to a better rupture risk stratification.

In previously published papers on patient-specific AAA wall stress analysis, CTA data without cardiac triggering were used for wall stress computations (Fillinger et al., 2002; Speelman et al., 2008; Truijers et al., 2007; Venkatasubramaniam et al., 2004). However, during imaging, the blood pressure results in a loaded state of the AAA. In all studies, this state is used as the stress free reference geometry, on which a patientspecific or population averaged systolic blood pressure is applied (Fillinger et al., 2002; Speelman et al., 2008; Truijers et al., 2007; Venkatasubramaniam et al., 2004). Clearly, this reference geometry is not stress free and any initial stresses present in the AAA wall are therefore discarded.

Raghavan et al. (2006b) previously proposed a method to approximate the unloaded AAA geometry by applying a backward deformation field, interpolated from the forward deformation field. The shape change of the AAA was assumed to be consistent for the forward and backward deformation. Lu et al. (2007) applied an inverse elastostatic method to obtain the zero-pressure geometry of preloaded biological structures. This method requires modifications in the finite element solution schemes, which is impossible for some commercial solvers. Although the stress with the proposed method was uniformly lower than with the conventional method, the stress distributions for both methods were found to be strongly similar, suggesting that the AAA shape was preserved (Lu et al., 2007).

The backward incremental (BI) method was proposed by de Putter et al. (2007), to account for the initial stress (IS) in arteries. Validation was done by showing a strong correspondence between the computed diastolic AAA geometry and the diastolic geometry determined from gated MR images, for three AAA's. They showed that the BI method leads to a stress free state for the load free geometry, which shows validity of the method. It was concluded by de Putter et al. (2007) that ignoring IS not only leads to an overestimation of the AAA volume, but also to an underestimation of the wall curvature and the wall stress. Hunter et al. (1989) identified local excrescences and 'blebs' in AAA's and discarding IS has the potential to AAA ballooning and flattening out these local 'blebs'.

The BI method was validated by de Putter et al. (2007), but no evaluation of the effect of initial stress incorporation (ISI) on the wall stress for different AAA's was performed. The stress analyses performed by de Putter et al. (2007) were also based 
on a linear elastic neo-Hookean material model and the differences between this model and the generally accepted nonlinear material model of Raghavan and Vorp (2000) were not investigated.

Computing AAA wall stresses without initial stress may have a different effect on the wall stress in each AAA. If that is the case, ISI is an important extension to the wall stress computations, as it leads to a more accurate wall stress prediction. However, if ignoring initial stresses has a uniform effect on all AAA wall stress results, the impact of ISI is small and may safely be discarded in future AAA wall stress computations. The aim of this study is to evaluate the effects of ISI on the patient-specific AAA wall stress and to evaluate the contribution of the nonlinear behavior of the AAA wall. Additionally, the effect of ISI on the local curvature is studied by evaluating the resulting AAA volumes and the average Gaussian curvatures.

\subsection{Methods}

CTA scans of 30 patients (AAA diameters 44-56 mm) were obtained from the databases of the Catharina Hospital in Eindhoven (the Netherlands, n=14) and the University Medical Center Maastricht $(n=16)$. Research approval was given by the Medical Ethics Commissions of the hospitals involved.

A standard contrast enhanced hospital protocol for AAA's was used (in-plane resolution 512x512 pixels, field-of-view 30-35 $\mathrm{cm}^{2}$, reconstructed slice thickness 1$2 \mathrm{~mm}$ ). Brachial systolic, diastolic and mean arterial blood pressures (SP, DP, MAP) were recorded with a Critikon Dynamap 1846SX/P, within 30 minutes after the CTA scan. The MAP was either obtained from the pressure measurements directly or, when not available, computed as $M A P=2 / 3 D P+1 / 3 S P$.

\subsubsection{Image Processing}

The CTA data was processed using an automatic segmentation procedure that has been developed by Philips Healthcare (Best, the Netherlands) in collaboration with University Medical Center Utrecht, Eindhoven University of Technology, Philips Healthcare Research (Paris, France) and our group (Breeuwer et al., 2008; de Putter et al., 2007; Delingette, 1994; Ollabarriaga et al., 2005). The segmentation procedure requires the placement of three user points in the flow lumen of the AAA, which are a starting point at the level of the renal arteries and two endpoints in both iliac arteries just distal to the aortic bifurcation. Subsequently, the centerline is automatically tracked by finding the minimal cost path between start and endpoints. A $3 \mathrm{D}$ active object (3DAO) is formed around the centerline and segmentation of the lumen and the wall is done by iteratively adapting the $3 \mathrm{DAO}$ to the boundaries of the lumen and thrombus, based on image features. Manual corrections were allowed by adapting slice contours when the automatic segmentation lacked accuracy. Further details on the segmentation process are described by Breeuwer et al. (2008).

Based on the wall segmentation, a 3D mesh of the AAA wall with a constant wall thickness of $2 \mathrm{~mm}$ was generated. The meshes typically consisted of 30,000 tetrahedral quadratic Crouzeix-Raviart elements. 
To determine the local curvature, the Gaussian curvature is estimated for each vertex on the outer surface of the models. For this purpose, a paraboloid is fitted to the vertex and its neighbors (Magid et al., 2007). The Gaussian curvature is computed based on the principal curvatures of the paraboloid and the average and standard deviation of the total Gaussian curvature was determined for each AAA.

\subsubsection{Finite Element Solution Process}

In the wall stress simulations, the AAA wall is considered as an incompressible solid. The deformation of an incompressible solid under a pressure load is described by the balance equations of mass and momentum. These are solved in combination with a constitutive equation, which describes the material behavior, and the boundary conditions, which comprehend the applied pressure and fixation of the upper and lower cross-sections of the AAA model. The commercial finite element package Sepran (Sepra, Delft, the Netherlands) is used to assemble and solve the resulting set of equations (see Appendix A for details). In an updated Lagrange formulation this yields a weak form according to de Putter et al. (2007):

$$
\left\{\begin{array}{c}
\int_{\Omega_{n-1}}\left(\nabla_{n-1} \boldsymbol{w}\right)^{T}: \boldsymbol{F}_{n-1, n}^{-1} \cdot \boldsymbol{\sigma}_{n} J_{n-1, n} \mathrm{~d} \Omega_{n-1}= \\
\int_{\Gamma_{n-1}} \boldsymbol{w} \cdot \boldsymbol{s}_{n} \tilde{J}_{n-1, n} \mathrm{~d} \Gamma_{n-1} \\
\int_{\Omega_{n-1}} q(J-1) J_{n-1, n} \mathrm{~d} \Omega_{n-1}=0
\end{array}\right.
$$

with the material law according to Raghavan and Vorp (2000):

$$
\boldsymbol{\sigma}_{n}=-p_{h} \boldsymbol{I}+2\left(\alpha+2 \beta\left(I_{B}-3\right)\right)\left(\boldsymbol{B}_{0, n}-\boldsymbol{I}\right)+2 \gamma\left(I I I_{B}-1\right) \boldsymbol{I}
$$

Here, $\Omega_{n-1}$ denotes the geometry, $\Gamma_{n-1}$ the boundary and $\nabla_{n-1}$ the gradient operator of the last known configuration $(n-1)$. The deformation tensor $\boldsymbol{F}_{n-1, n}$ defines the deformation between $\Omega_{n-1}$ and the current geometry $\Omega_{n}$. $\boldsymbol{w}$ and $q$ are weighting functions and $\boldsymbol{\sigma}_{n}$ is the Cauchy stress tensor. $J_{n-1, n}=\operatorname{det}\left(\boldsymbol{F}_{n-1, n}\right)$ and $\widetilde{J}_{n-1, n}$ are the relative change in volume and surface area between $\Omega_{n-1}$ and $\Omega_{n}$, i.e. $d \Omega_{n}=J_{n-1, n} d \Omega_{n-1}$ and $d \Gamma_{n}=\tilde{J}_{n-1, n} d \Gamma_{n-1}$. The surface traction $\boldsymbol{s}_{n}=p_{l, n} \boldsymbol{n}$ is defined by the boundary condition in terms of a pressure load $p_{l, n}$.

In the constitutive equation describing the material behavior, $\boldsymbol{I}$ is the identity matrix, $\boldsymbol{B}$ the Finger tensor, defined as $\boldsymbol{B}_{0, n}=\boldsymbol{F}_{0, n} \cdot \boldsymbol{F}_{0, n}^{T}=\boldsymbol{F}_{n-1, n} \cdot \boldsymbol{B}_{0, n-1} \cdot \boldsymbol{F}_{n-1, n}^{T}$, and $I_{B}$ and $I I I_{B}$ are the first and third invariants of $\boldsymbol{B}_{0, n}$. Also, $p_{h}$ is the hydrostatic pressure, and $\alpha, \beta$ and $\gamma$ are the material parameters.

Given the state of stress and deformation at load case $(n-1)$, the nonlinear algebraic system, obtained after deriving a finite element representation of the weak form given above, is linearized with Newton's method and iteratively solved using an incomplete LU preconditioned Bi-CGSTAB method. For the complete finite element formulation of the balance laws, see Appendix A. 
In general the incremental process summarizes to:

$$
\left[\boldsymbol{F}_{n-1, n}, \delta \boldsymbol{\sigma}_{n}\right]=f\left(\Omega_{n-1}, \boldsymbol{F}_{0, n-1}, \boldsymbol{\sigma}_{n-1}, p_{l, n}, \alpha, \beta, \gamma\right) .
$$

The updates in the deformation $\boldsymbol{F}_{n-1, n}$ and stress $\delta \boldsymbol{\sigma}_{n}$ in load case $n$ thus depend on $\Omega_{n-1}, \boldsymbol{F}_{0, n-1}$ and $\boldsymbol{\sigma}_{n-1}$ in load case $(n-1)$, the pressure load $p_{l, n}$, and the material parameters $\alpha, \beta$ and $\gamma$.

The balance equations are solved in two incremental pressure load stages. Stage 1 is the backward incremental (BI) stage and runs from $0 \mathrm{kPa}$ to the patient-specific mean arterial pressure (MAP) $p_{l, r}$. Stage 2 is performed conventionally and runs from the MAP to a systolic pressure $p_{l, s}$ of $18.7 \mathrm{kPa}(140 \mathrm{mmHg})$, which is the same for all AAA's to facilitate comparison between the results of the patients mutually. For both stages, 16 incremental steps were used to apply the pressure starting from the measured reference geometry $\Omega_{r}$ :

$$
\begin{aligned}
& \Omega_{0}=\Omega_{r} \\
& \boldsymbol{F}_{0,0}=\boldsymbol{I} \\
& \boldsymbol{\sigma}_{0}=\mathbf{0} \\
& \text { for } n=1, N: \\
& \quad\left[\boldsymbol{F}_{n-1, n}, \delta \boldsymbol{\sigma}_{n}\right]=f\left(\Omega_{n-1}, \boldsymbol{F}_{0, n-1}, \boldsymbol{\sigma}_{n-1}, p_{l, n}, \alpha, \beta, \gamma\right) \\
& \quad \text { if }(\text { stage }==1) \text { then } \Omega_{n}=\Omega_{n-1} \\
& \quad \text { if }(\text { stage }==2) \text { then } \Omega_{n}=\Omega_{n}\left(\boldsymbol{F}_{n-1, n}, \Omega_{n-1}\right) \\
& \quad \boldsymbol{F}_{0, n}=\boldsymbol{F}_{0, n-1} \cdot \boldsymbol{F}_{n-1, n} \\
& \quad \boldsymbol{\sigma}_{n}=\boldsymbol{\sigma}_{n-1}+\delta \boldsymbol{\sigma}_{n} \\
& \text { endfor }
\end{aligned}
$$

Here, the only difference between stages 1 and 2 is that in the BI stage, no update of the geometry is performed. Therefore, in general, no equilibrium exists in the beginning of each iteration. However, the final pressure steps in stage 1 are chosen small enough to ensure equilibrium at the end of stage 1 . In this way, $\boldsymbol{F}_{0, n}=$ $\boldsymbol{F}_{0, n-1} \cdot \boldsymbol{F}_{n-1, n}$ implicitly updates the load free configuration and $\boldsymbol{\sigma}_{n}=\boldsymbol{\sigma}_{n-1}+\delta \boldsymbol{\sigma}_{n}$ updates the initial stress. Once in stage 1 equilibrium has been reached, the convexity of the constitutive equations ensures a unique initial stress state at the reference configuration $\Omega_{r}$ and a unique load free and stress free configuration $\Omega_{0}$.

The parameters $\alpha$ and $\beta$ in the constitutive equation are set to $0.17 \mathrm{MPa}$ and $1.9 \mathrm{MPa}$ (Raghavan and Vorp, 2000), while the stabilization parameter $\gamma$ is set to $0.5 \mathrm{MPa}$. For the linear material model, $\alpha=0.45 \mathrm{MPa}$ and $\beta=0 \mathrm{MPa}$.

\subsubsection{Data Analysis}

The wall stress for all AAA's is computed with initial stress incorporation (ISI) and with the assumption that initial stress can be omitted (ISO). Wall stress results are presented, together with the AAA volumes and average Gaussian surface curvature at zero, mean arterial and peak systolic pressure. Thereafter, the 99-percentile wall stress was used as single stress parameter (Speelman et al., 2008). The 99-percentile stress is computed as the peak maximum principal stress after excluding $1 \%$ of the 
wall with the highest stress. We have previously shown that the 99-percentile stress has a much higher reproducibility than the peak wall stress (Speelman et al., 2008, see chapter 2).

The 99-percentile wall stress is computed for all 30 AAA's with ISO and ISI, with the linear (LM) and nonlinear material model (NM). Linear regression analyses were performed between the wall stress results of the four different combinations (ISI/ISO$\mathrm{LM} / \mathrm{NM}$ ). For these regression models, the vertical intercept was forced at $0 \mathrm{kPa}$.

The maximum AAA diameter was determined from the CTA data as the largest in-plane anterior-posterior AAA diameter. The relation between the 99-percentile stress and the maximum AAA diameter was evaluated for each combination ISI/ISOLM/NM. An average regression model was determined and the AAA's with relative high or low stress, than estimated by the diameter alone, were identified. Therefore, the residuals of the regression models were determined and normalized with the standard deviation. In this way, the relative position of each AAA with regard to the average regression model could be compared between the four combinations. The statistical evaluation was performed with Statgraphics Centurion XV (StatPoint, Herndon, VA, USA).

\subsection{Results}

Figure 3.1 displays the stress distributions for one AAA at different pressure states. The top left image displays the pressure stretch curve for an artery with nonlinear material behavior with matching cross-sections. The left line is the relation that is experienced in-vivo by the artery $(B$ to $D)$. When the segmented geometry $\left(C_{1}\right)$ is used as reference geometry without ISI, the right curve is followed $\left(C_{1}\right.$ to $\left.E\right)$. With ISI, $C_{2}$ is reached with exactly the same geometry as $C_{1}$, only now with initial stress in the wall. From $C_{2}$, the pressure can either be increased to a systolic pressure $(D)$ or decreased to zero $\mathrm{mmHg}(A)$. The stress free geometry $A$ is smaller than $C_{1}$ (volume $119 \mathrm{ml}$ versus $123 \mathrm{ml}$ ). When comparing $D$ and $E$, we see that with ISI, a smaller systolic geometry is obtained than without ISI (volume $125 \mathrm{ml}$ versus $130 \mathrm{ml}$ ), with different stress distributions. The 99-percentile stress for geometry $E$ is $281 \mathrm{kPa}$ and $347 \mathrm{kPa}$ for geometry $D$.

\subsubsection{Generic Effects}

The average Gaussian curvature ( \pm standard deviation) for all AAA's in the reference state was $0.46 \pm 0.10$. Although not significant (p-value 0.26), the average Gaussian curvature of the systolic geometry computed without ISI was found to be lower $(0.49 \pm 0.11)$ than with ISI $(0.53 \pm 0.10)$. Both curvatures without and with ISI are slightly higher than in the reference state, but not significant (p-values 0.63 and 0.16$)$.

The average volume of the AAA's in the reference state was $139 \pm 45 \mathrm{ml}$. At zero pressure, the volume was on average $3 \%$ lower (range 1-4\%), while the volume was $6 \%$ larger at systolic pressure without ISI (range 4-10\%) and 2\% with ISI (range 1-4\%). The volume overestimation at systolic pressure with ISO was $4 \%$ (range 2-6\%). 


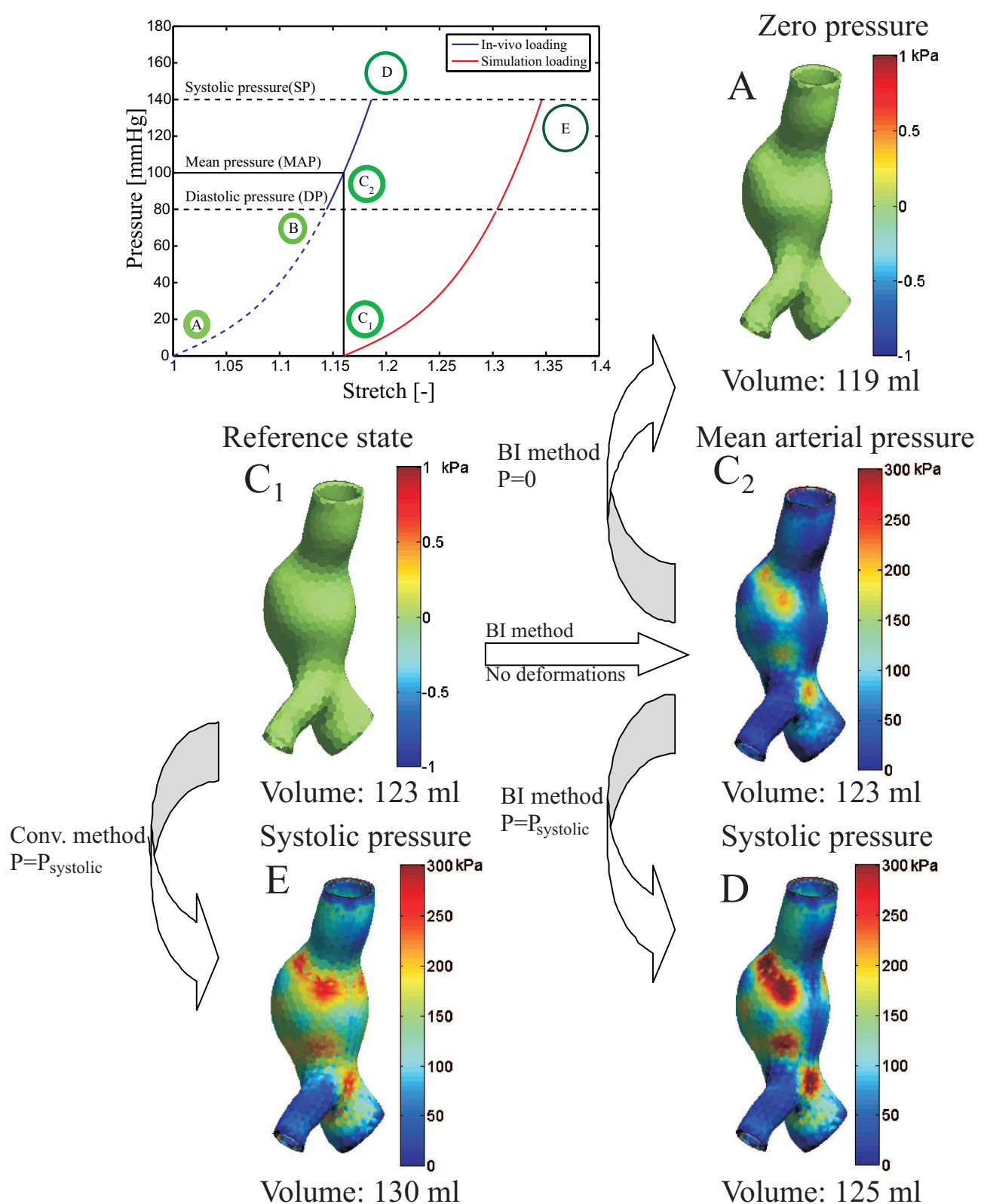

Figure 3.1: Wall stress distributions for one $A A A . C_{1}$ represents the measured reference state. $C_{2}$ represents the mean arterial pressure state as computed with ISI. $A$ and $D$ are respectively the unloaded and systolic pressure state. E represents the systolic pressure state as computed with the conventional ISO method. 
Figure 3.2 shows the linear regression models between the 99-percentile stress for all AAA's as computed by the four combinations of ISI/ISO-LM/NM mutually. All relations show a strong linear relation $\left(R^{2} 0.77-0.95\right)$. When ISO-NM is used instead of the ISO-LM, stresses are on average 18\% higher (figure 3.2A). For ISI, this is $40 \%$ (figure 3.2C). Stresses are on average $21 \%$ higher for ISI-LM than for ISO-LM (figure $3.2 \mathrm{~B}$ ). For NM, this is $42 \%$ (figure $3.2 \mathrm{D}$ ).
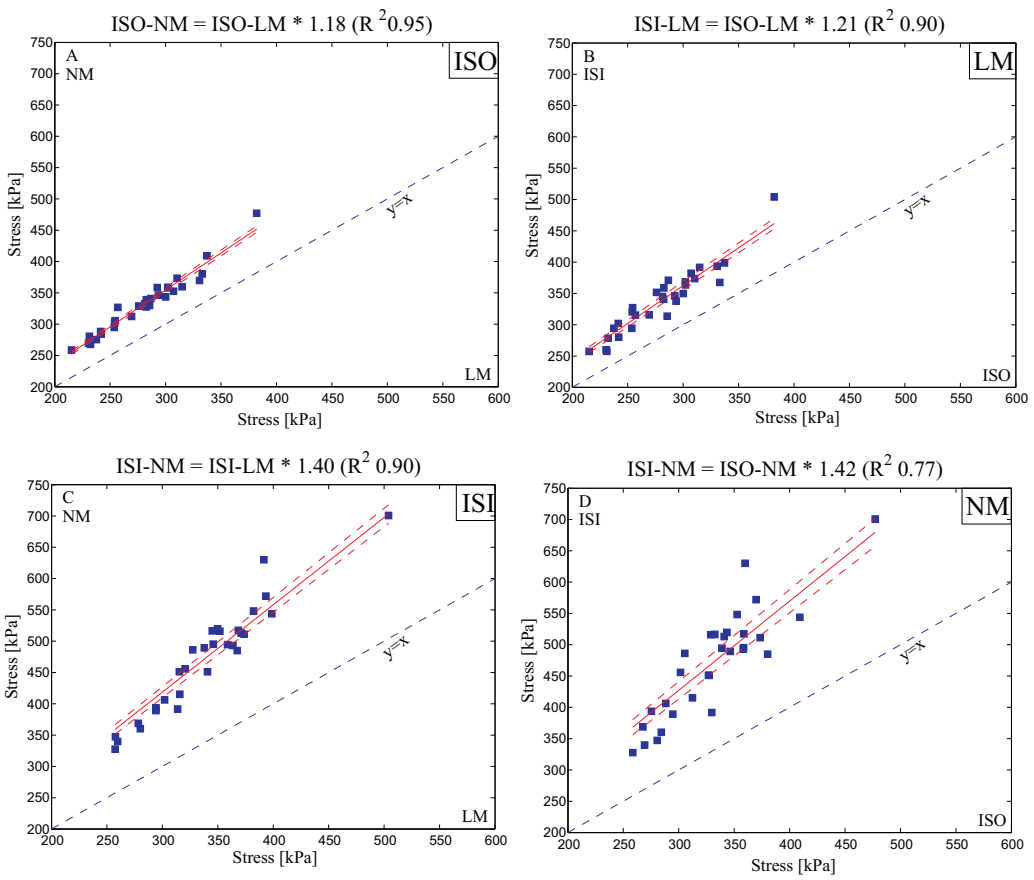

Figure 3.2: Linear regression for A) the ISO model using the linear (LM) and nonlinear (NM) material model; B) LM using ISO and ISI; C) ISI using LM and NM; D) NM using ISO and ISI. The dashed lines indicate one standard deviation of the regression model.

\subsubsection{Patient-Specific Effects}

Figure 3.3 (left) shows the relation between the maximum AAA diameter with the 99-percentile stress using ISI-NM. The dashed lines bound the interval of 1 standard deviation (SD) of the residuals of the regression model. AAA's 1, 11, 14, 28 and 30 have stresses more than 1 SD above the average regression model and AAA's 8, 9, 15, 17, 20 and 26 have stresses more than 1 SD below the model. This can also be seen in the right image of figure 3.3, where the residuals of the model are given, normalized by the SD. 
The regression models between the maximum diameters and the wall stresses, as computed with the four combinations ISI/ISO-LM/NM, all showed a significant positive linear relation. In figure 3.4, the normalized residuals are plotted for the four combinations. It shows that depending on the combination of computational method (ISI/ISO) and material model (LM/NM), an AAA can have a 99-percentile stress more than $1 \mathrm{SD}$ above the average model using one combination, whilst it has an average stress when using another (for instance, see AAA's 10-13, 22, 23 and 28). This is also seen for AAA's with a relative low stress (more than 1 SD below the average model, see AAA's 4, 9, 17 and 25).
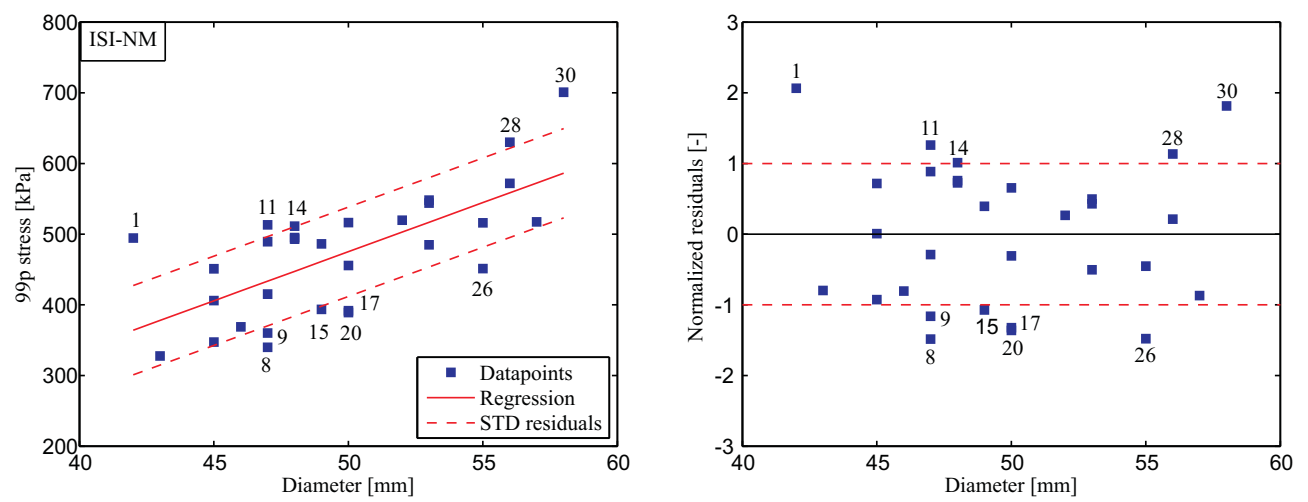

Figure 3.3: The regression model between the maximum diameter and the 99percentile stress as determined with ISI-NM (left). The normalized residuals of the regression model (right).

\subsection{Discussion}

Patient-specific AAA wall stress analyses are currently performed by several researchers, with the goal to improve rupture risk assessment (Fillinger et al., 2002; Speelman et al., 2008; Truijers et al., 2007; Venkatasubramaniam et al., 2004). Most researchers utilize a nonlinear material model to simulate AAA wall behavior. However, the initial stress (IS) that is present in the AAA wall during imaging is usually neglected.

In this study the BI method was applied to thirty AAA's to incorporate initial stress (ISI). Figure 3.1 shows the geometries and wall stress distributions at zero, mean arterial and systolic pressure for one AAA. Without ISI, a larger volume increase $(+6 \%)$ due to the systolic pressure was observed than computed with ISI $(+2 \%)$. Also a slightly lower average Gaussian curvature (not significant, p-value 0.26) was found when ISI was not applied. Although the AAA's were clearly more inflated without ISI, the differences in Gaussian curvature were too small to state that local 'blebs' (Hunter et al., 1989) were more flattened without ISI. However, the wall stress 


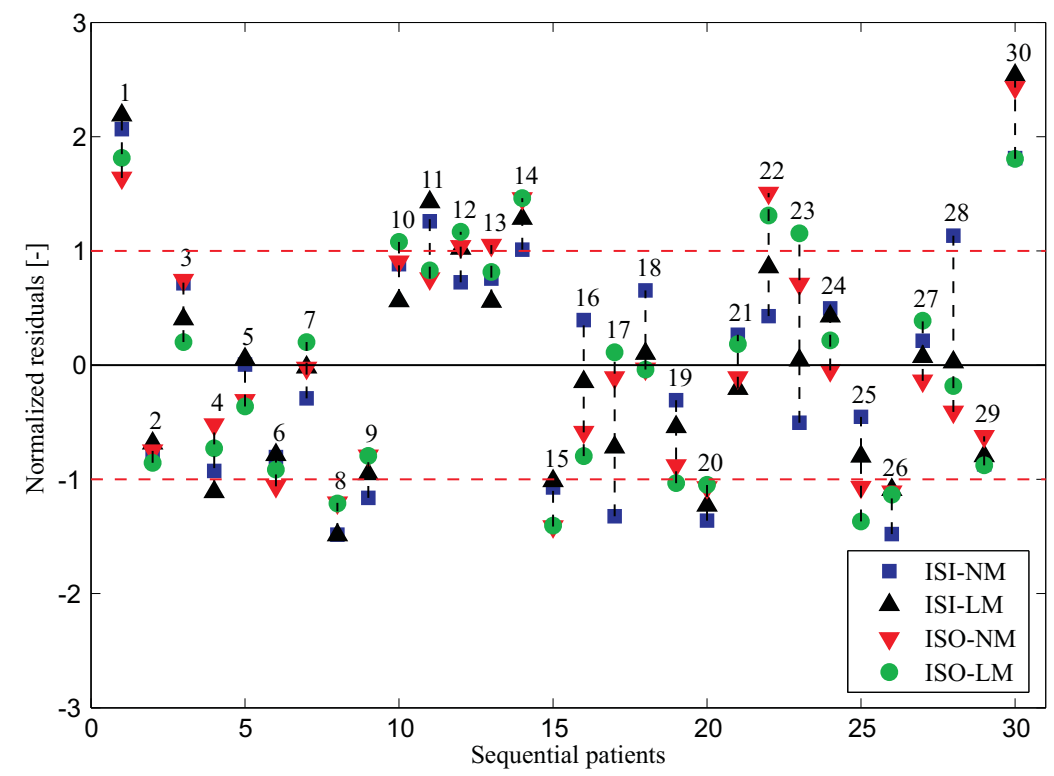

Figure 3.4: The normalized residuals for the four combinations of computational method and material model (ISI/ISO-LM/NM).

distributions at systolic pressure were found to be markedly altered with ISI (figure 3.1 ), suggesting (small) local changes in the AAA geometry. This is in contrast with the results from Lu et al. (2007), who found no differences in wall stress distributions when IS was incorporated.

Figure 3.2 shows the generic effects that material nonlinearity and initial stress have on the wall stress of all AAA's in this study. The increase due to nonlinearity of the AAA wall behavior may be considered uniform for all AAA's (18\% for ISO, $40 \%$ for ISI, $R^{2} 0.95$ and 0.90 respectively). Computations with ISI also resulted in a stress increase by $21 \%\left(\mathrm{LM}, R^{2} 0.90\right)$ and $42 \%\left(\mathrm{NM}, R^{2} 0.77\right.$ ). The relative low regression coefficient of 0.77 indicates that incorporating IS affects the wall stress differently for each patient.

A significant positive relation was found between the maximum AAA diameter and the resulting wall stress, for all four combinations (ISI/ISO-LM/NM, figure 3.3). Of particular interest are the AAA's that do not follow the average regression model, but lie vast above or below the average relation. AAA's that have a significant higher wall stress than expected based on the diameter, may experience a faster growth and higher rupture risk and may require a more stringent follow-up plan.

The results show that the relative position of the AAA's with respect to the average regression model depends on the combination of computational method and material model used in the wall stress simulations (figure 3.4). For instance, patient 23 has a wall stress more than $1 \mathrm{SD}$ above the average model for ISO-LM. However, for ISI-NM, the stress is even below average. For patient 28 , the opposite can be seen; 
whereas for ISI-NM the stress is more than $1 \mathrm{SD}$ above the regression model, the stresses are more or less average for the other computations. The group of patients with relative high AAA wall stress thus changes, when the initial stress and material nonlinearity are neglected.

Some limitations in this study need to be mentioned. First of all, although IS was incorporated in the analyses, residual stress in the AAA wall was not accounted for. Residual stress can globally be estimated using the opening angle of an artery (Alastrué et al., 2007). Unfortunately, no methods are currently available to compute the local residual stress in-vivo or to estimate opening angles of in-vivo arteries.

Intraluminal thrombus and calcifications are not incorporated in the current wall stress study. Thrombus is found to be a very soft material (Ashton et al., 2009; van Dam et al., 2008), and likely has a negligible effect on the resulting wall stress. Calcifications will have a significant effect on the local wall stress (Speelman et al., 2007), but are difficult to include as the material properties and the interaction between AAA wall and calcifications remain unknown.

\subsection{Conclusions}

The current study shows that, when initial stress and material nonlinearity are omitted in AAA wall stress analyses, different effects on the wall stress are found for each patient. Therefore, provided that other assumptions made in this work are not predominant, initial stress and material nonlinearity should be accounted for in future AAA wall stress computations. 
Chapter 3 


\section{Chapter 4}

\section{The Mechanical Role of Thrombus on the Growth Rate of an AAA}

This study investigates the effect of intraluminal thrombus on the wall stress and growth rate of aneurysms, using both idealized and patient-specific AAA models in wall stress computations.

The reduction in wall stress for a range of thrombus volumes and shear moduli was computed using idealized AAA models. For thirty patient-specific AAA models with varying thrombus volumes, the wall stress was computed with and without thrombus. The AAA growth rate was compared for AAA's with a small and large thrombus volume. The results were compared between the idealized and patient-specific models. AAA's with a large thrombus were found to have a significant stronger growth in diameter than aneurysms with a small thrombus (p-value $<0.01)$. The stress reduction due to the thrombus showed the same trend for the idealized and patientspecific models, although the effect was underestimated by the idealized models. Also, a considerable variation between patients was observed.

Due to the variation between patients, the effect of thrombus can only be determined by patient-specific wall stress analysis. However, as AAA's with large thrombi tend to grow faster, weakening of the AAA wall, under the influence of thrombus, may play a more imminent role in the process of AAA growth, than the wall stress.

This chapter is based on: L. Speelman, G. W. Schurink, E. M. Bosboom, J. Buth, M. Breeuwer, F. N. van de Vosse and M. J. Jacobs, The mechanical role of thrombus on the growth rate of an AAA, J. Vasc. Surg. 2009, in press. 


\subsection{Introduction}

In the decision for surgical repair of abdominal aortic aneurysms (AAA's), the risk of rupture is weighed carefully against the risk of the surgical procedure. Currently, the maximum AAA diameter is the main factor that determines the decision for surgery. However, the rupture risk is obviously dependent on a complex combination of both generic as well as patient-specific factors. In the past, several studies have concluded that the diameter may not be reliable as rupture risk criterion for the individual patient and that it should be replaced by a more patient-specific criterion (Darling et al., 1977; Nicholls et al., 1998; Vorp and vande Geest, 2005). Therefore, AAA wall stress studies have been introduced, which include the complete AAA geometry in the AAA risk estimation. It was previously shown that AAA wall stress has higher sensitivity and specificity than the maximum diameter in this risk estimation (Fillinger et al., 2002). Additionally, it was found that peak wall stress was higher for ruptured than for non-ruptured or asymptomatic AAA's (Truijers et al., 2007; Venkatasubramaniam et al., 2004).

Intraluminal thrombus is found in the majority of the AAA's (Harter et al., 1982) and the size of the thrombus varies strongly between patients. The volume and thickness of thrombus have previously been indicated to influence the growth rate (Wolf et al., 1994) and the rupture risk (Hans et al., 2005; Satta et al., 1996) of AAA's. Several computational studies have focused on thrombus in the past in either idealized (di Martino and Vorp, 2003; Mower et al., 1997) or patient-specific geometries (Doyle et al., 2007; Li et al., 2008; Wang et al., 2002). All studies concluded that thrombus significantly lowers AAA wall stress and that the effect was stronger for thicker and stiffer thrombi. Recently, material properties of thrombus were reevaluated with compression and shear experiments (Ashton et al., 2009; Hinnen et al., 2007; van Dam et al., 2008), leading to lower shear moduli than was determined previously (di Martino et al., 1998; Gasser et al., 2008; vande Geest et al., 2006b; Wang et al., 2001).

The aim of this study is to evaluate the effect of thrombus on the wall stress in patientspecific AAA models, in the light of the recently obtained material properties. The results will be compared with the results of an idealized axisymmetric AAA model, using a range of thrombus elastic moduli and relative thrombus volumes. Thrombus will be modeled as an incompressible linear elastic material, which is fixed to the aneurysm wall. Demographical and geometrical characteristics, diameter growth, and wall stress results are compared between AAA's with small and large thrombus volumes.

\subsection{Methods}

\subsubsection{Idealized Models}

An idealized axisymmetric finite element model of an AAA was created with a maximum diameter of $5 \mathrm{~cm}$ and a diameter of $2 \mathrm{~cm}$ at the proximal and distal ends. The wall thickness was kept constant on $2 \mathrm{~mm}$. In the models where thrombus was 
incorporated, an overall minimum thrombus thickness of $2 \mathrm{~mm}$ was applied to each model, and the maximum thrombus thickness was set to 2, 4, 6, 8, 10 and $12 \mathrm{~mm}$, located at the widest section of the model (figure 4.1). This resulted in relative thrombus volumes of $18,28,36,44,51$ and $57 \%$.

Quadratic hexahedral elements were used for both the thrombus and the wall and mesh independency was reached for all models. The nonlinear material model as proposed by Raghavan and Vorp (2000) has been applied to the AAA wall. Linearization of this material model around a physiological pressure would result in a shear modulus of about $900 \mathrm{kPa}$. The thrombus was fixed to the aneurysm wall and modeled as an incompressible linear elastic material. Table 4.1 summarizes the shear moduli $(\mathrm{G})$ as found in literature for thrombus (Ashton et al., 2009; di Martino et al., 1998; Gasser et al., 2008; Hinnen et al., 2007; van Dam et al., 2008; vande Geest et al., 2006b; Wang et al., 2001). In the model, no distinction was made between luminal, medial or abluminal thrombus. The shear moduli applied to the thrombus in the idealized model were 4, 10, 40, 100 and $180 \mathrm{kPa}$, covering the gross range of the found moduli (table 4.1).

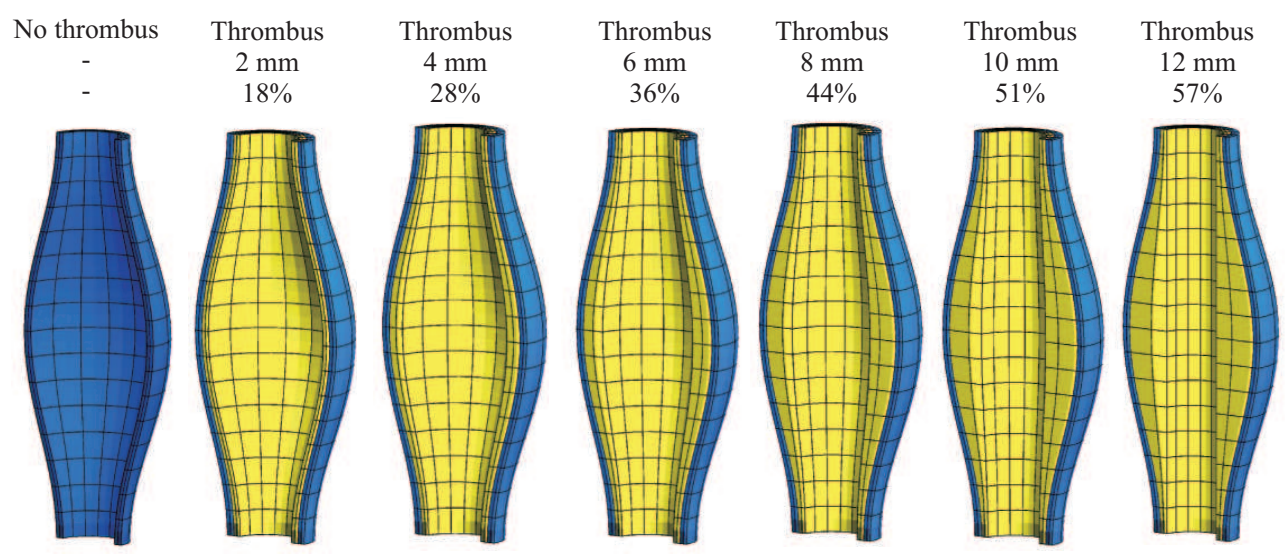

Figure 4.1: The idealized axisymmetric $A A A$ models without and with thrombus, with a minimum layer of $2 \mathrm{~mm}$ thrombus and a maximum thrombus thickness ranging from $2-12 \mathrm{~mm}$ (18-57\% relative thrombus volume).

\subsubsection{Patient-Specific Models}

Contrast enhanced CTA data of 30 patients with a medium-sized AAA (maximum diameter between 4.0 and $5.5 \mathrm{~cm}$ ) were obtained in the Catharina Hospital Eindhoven (the Netherlands, $\mathrm{n}=14$ ) and the University Medical Center Maastricht $(\mathrm{n}=16)$. A standard contrast enhanced hospital protocol for AAA's was used (in-plane resolution $512 \times 512$ pixels, field-of-view 30-35 $\mathrm{cm}^{2}$, reconstructed slice thickness $1-2 \mathrm{~mm}$ ). Only patients with at least one sequential CTA scan after the one used for the analysis were included in the study, to determine the prospective growth rate of the AAA. All 
patients signed informed consent and research approval was given by the local Medical Ethics Commissions. Patient demographical information was collected at the time of inclusion. All CTA's were performed in the arterial phase (in-plane resolution $512 \times 512$, maximum slice thickness $2 \mathrm{~mm}$ ). Brachial systolic, diastolic and mean arterial pressures (SP, DP and MAP) were recorded using a Dynamap 1846SX/P (Critikon Inc, Tampa, FL, USA) within 30 minutes after the CTA scan.

Table 4.1: Literature values (mean \pm standard deviation) for the shear modulus $(G)$ for luminal, medial and abluminal thrombus.

\begin{tabular}{lccc}
\hline \hline & Test & Thrombus & G (kPa) \\
\hline di Martino et al. (1998) & Uniaxial stretching & luminal & $44 \pm 21$ \\
Wang et al. (2001) & Uniaxial stretching & luminal & $180 \pm 23$ \\
& & medial & $90 \pm 20$ \\
vande Geest et al. (2006b) & Biaxial stretching & luminal & $67 \pm 5$ \\
Hinnen et al. (2007) & Shear loading & - & $12 \pm 5$ \\
van Dam et al. (2008) & Shear loading & - & $1.7 \pm 1.3^{*}$ \\
Gasser et al. (2008) & Uniaxial stretching & luminal & $21 \pm 6^{*}$ \\
& & medial & $16 \pm 5^{*}$ \\
& & abluminal & $14 \pm 5^{*}$ \\
Ashton et al. (2009) & Compression & luminal & $0.5 \pm 0.1$ \\
& & medial & $0.8 \pm 0.2$ \\
& & abluminal & $6.4 \pm 1.8$ \\
\hline
\end{tabular}

* Reported values, otherwise derived from the elastic modulus $\mathrm{E}(G=1 / 3 E)$

\subsubsection{AAA Segmentation}

The geometry of the AAA was segmented from the CTA data using software developed by Philips Healthcare (Best, the Netherlands) in collaboration with University Medical Center Utrecht, Eindhoven University of Technology, Philips Healthcare Research (Paris, France) and our group (Breeuwer et al., 2008; de Putter et al., 2007; Delingette, 1994; Ollabarriaga et al., 2005). A start point in the flow lumen at the level of the renal arteries and two end points just distal to the aortic bifurcation needed to be selected by the user. The centerline of the AAA was tracked automatically between the three points by means of a minimal cost path approach (Breeuwer et al., 2008). A 3D active object (3DAO) was formed around the centerline and iteratively adapted to the boundaries of the flow lumen, based on grey value thresholds in the CTA images (Delingette, 1994). Thereafter, the boundary of the thrombus was found by iteratively adapting the 3DAO using a profile force method (Ollabarriaga et al., 2005). Manual corrections of the surfaces were allowed by adapting the slice contours when the user felt that the automatic segmentation lacked accuracy. A minimum distance of $2 \mathrm{~mm}$ between the lumen surface and thrombus surface was set to prevent crossing of both surfaces. Around the thrombus, a third surface was created, placed $2 \mathrm{~mm}$ outward (AAA wall, see figure 4.2). 
A finite element model was created of the thrombus and AAA wall, by filling the space between two surfaces with 15-node quadratic tetrahedral Crouzeix-Raviart elements. The thrombus mesh is formed between the lumen and thrombus surface, resulting in a layer of thrombus throughout the whole AAA, with a minimum thickness of $2 \mathrm{~mm}$. The AAA wall mesh is formed between the thrombus and the wall surface, leading to a constant wall thickness of $2 \mathrm{~mm}$. The size of the elements was kept constant and, depending on the mesh volume, the number of elements per AAA varied between 5,000 and 10,000 for the wall and 6,000 to 20,000 for the thrombus. A sensitivity study showed that mesh independency was reached for this mesh size as stresses changed less than $1 \%$ for smaller elements.

A nonlinear hyper-elastic material model was applied to the wall (Raghavan and Vorp, 2000). The thrombus was fixed to the AAA wall and modeled with an incompressible and isotropic linear material model with a shear modulus of $40 \mathrm{kPa}$, representing the average value from table 4.1 (Ashton et al., 2009; di Martino et al., 1998; Gasser et al., 2008; Hinnen et al., 2007; van Dam et al., 2008; vande Geest et al., 2006b; Wang et al., 2001). Due to the complex geometries, lower shear moduli for the thrombus gave numerical instabilities and are therefore only applied to the idealized AAA model.
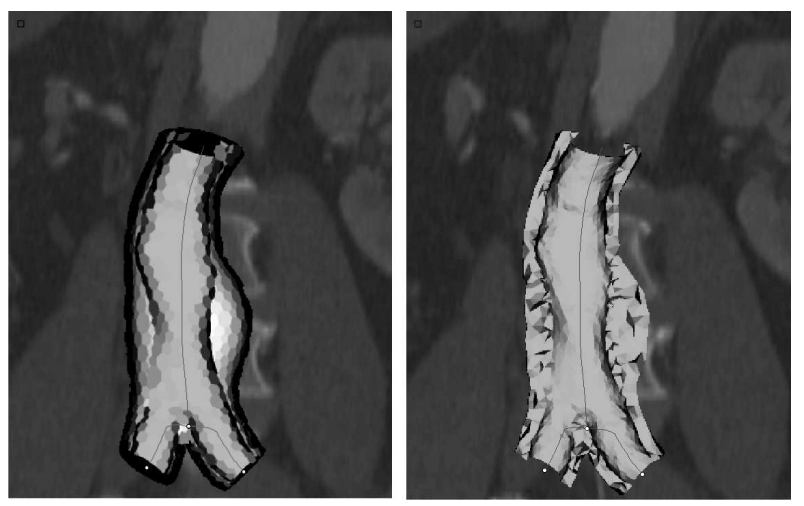

Figure 4.2: The surfaces of the lumen (grey), the thrombus (white) and the $A A A$ wall (black) displayed in the CTA scan(left). The resulting finite element mesh (right).

\subsubsection{Geometrical Characteristics}

Stereolithographic (STL) data of the lumen, thrombus and wall volumes were created automatically by the segmentation software. From the STL data, all volumes were determined and the relative thrombus volume as a percentage of the whole AAA volume was computed. The iliac arteries were excluded from the volume measurements. In addition, the maximum thrombus thickness and the maximum anterior-posterior AAA diameter were determined from the CTA data. The tortuosity of the AAA between the renal arteries and the aortic bifurcation was computed for 
both the flow lumen and complete AAA geometry. For this, the length of respectively the central flow and AAA line is divided by the straight distance between start and endpoint (Chaikof et al., 2002; Speelman et al., 2008). The annual growth rate was linearly extrapolated from the difference in maximum AAA diameter in the CTA scan used for analysis and the last available CTA scan.

\subsubsection{AAA Wall Stress Analysis}

The finite element software Sepran (Sepra, Delft, the Netherlands) was used to calculate the AAA wall stresses with and without thrombus, for both the idealized and the patient-specific AAA models. The wall stress computations were based on conservation of mass and momentum (second law of Newton) for all finite elements in the model. Boundary conditions were required to solve the complex system of conservation laws. These comprehend, in addition to the applied pressure, complete fixation of the most proximal and distal planes of the models. For the finite element formulation of the balance laws, see Appendix A.

A patient group average systolic blood pressure of $18.7 \mathrm{kPa}(140 \mathrm{mmHg})$ was applied to the inner surface of either the wall or, when present, the thrombus. During CTA image acquisition, a mean arterial pressure (MAP) was present in the AAA, leading to a certain initial stress configuration. This initial stress was accounted for by applying the backward incremental method in the wall stress computations (de Putter et al., 2007; Speelman et al., 2009a, see chapter 3). The patient-specific MAP as measured directly after the CTA scan was used to compute the initial stress.

Maximum principal stresses, strains and pressure were computed in all nodes of the finite element models. As the peak wall stress showed to be highly sensitive to small geometrical variations introduced by the segmentation and meshing process, the 99percentile stress was used as wall stress measure for each AAA (Speelman et al., 2008, see chapter 2). The 99-percentile stress is the highest maximum principal stress in the AAA, after exclusion of $1 \%$ of the nodes containing the highest stresses.

\subsubsection{Data Analysis}

Using the idealized AAA models, the effect on the 99-percentile wall stress was determined for a range of relative thrombus volumes (18-57\%) and shear moduli $(4-180 \mathrm{kPa})$. For the patient-specific AAA models, the decrease in wall stress due to the thrombus, was related to the thrombus volume and compared to the results from the idealized models.

The patient-specific AAA models were divided over two groups, based on their relative thrombus volume $(<$ and $>$ median). ANOVA tests were performed to test for significant differences in demographical, geometrical, and biomechanical characteristics between both groups. A p-value $<0.05$ was considered significant. The statistical evaluation was done with Statgraphics Centurion XV (StatPoint, Herndon, VA, USA). 


\subsection{Results}

\subsubsection{Idealized Models}

Figure 4.3 shows the change in 99-percentile wall stress due to the presence of thrombus, compared to the wall stress without thrombus, as computed with the idealized AAA model. The response in relation to the shear modulus appears to be nonlinear; the effect of thrombus on the wall stress is less sensitive for an change in shear modulus at higher shear moduli. For the model with a uniform thrombus layer of $2 \mathrm{~mm}$, the effect with a thrombus shear modulus of $4 \mathrm{kPa}$ is only $2 \%$, whereas for $40 \mathrm{kPa}$ this is $7 \%$, and for $180 \mathrm{kPa}$ this is $25 \%$.

The decrease in wall stress in relation to the relative thrombus volume is close to linear for all shear moduli. For a thrombus shear modulus of $4 \mathrm{kPa}$, a thrombus with relative volume of $30 \%$ results in a $6 \%$ decrease, whereas this is $12 \%$ for a relative thrombus volume of $57 \%$. For a shear modulus of $40 \mathrm{kPa}$, the wall stress is decreased $18 \%$ and $35 \%$, respectively.
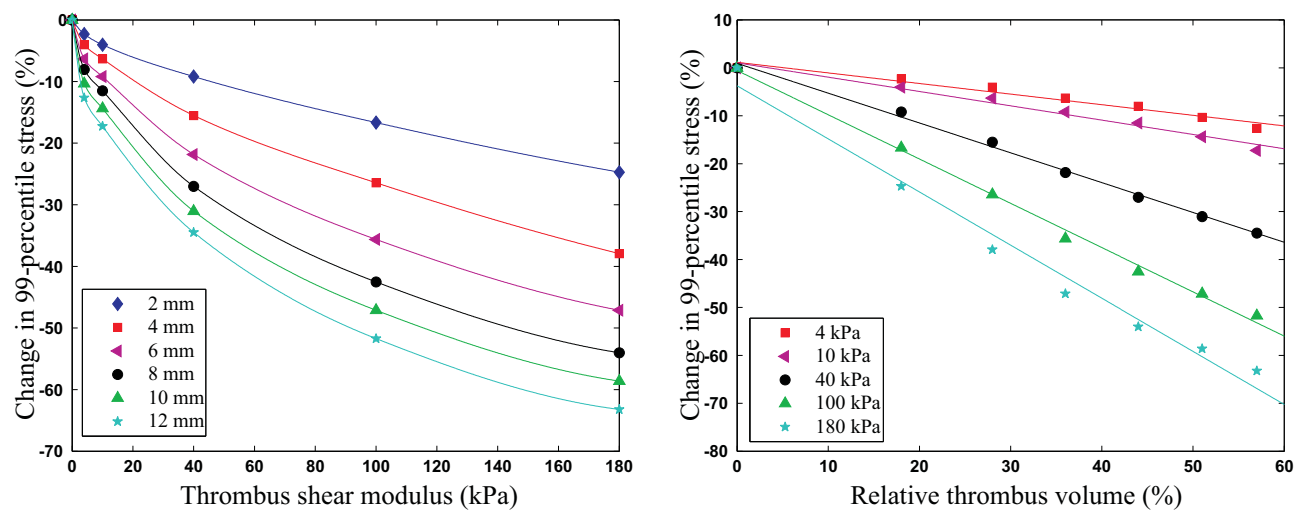

Figure 4.3: The change in wall stress due to the presence of thrombus as a function of the thrombus shear modulus for a thrombus thickness 2-12 $\mathrm{mm}$ (left) and as a function of the relative thrombus volume for thrombus shear moduli of $4-180 \mathrm{kPa}$ (right).

\subsubsection{Patient-Specific Models}

\section{AAA Characteristics}

The median relative thrombus volume for the 30 patients was $32 \%$. Table 4.2 summarizes the AAA characteristics of the two groups with a relative thrombus volume below (group $\mathrm{A}, \mathrm{n}=15$ ) and above median (group $\mathrm{B}, \mathrm{n}=15$ ). There was no significant difference in age, gender, hypertension, smoking, diabetes mellitus and statin-use between groups A and B. Besides the maximum diameter and the total 
AAA volume, also the tortuosities and the 99-percentile wall stress without thrombus matched for both groups. The average thrombus volume of group B was larger than for group A (p-value $<0.01)$, therefore, the lumen volume was larger in group A (p-value $<0.01)$. Also, the maximum thrombus thickness was larger for group B $(\mathrm{p}$-value $<0.01)$.

The mean follow-up time of the patients was 9 months (range 4-12 months). The median (quartiles) growths in AAA diameter were 0 (0-1.2) and 3 (1-6) mm for groups $\mathrm{A}$ and $\mathrm{B}$. The distributions in the two groups differed significantly (Kruskal-Wallis test, p-value $<0.01)$. Figure 4.4 shows the box-and-whisker plots for both groups. The wall stress as computed with thrombus was significantly lower for group B (pvalue $<0.01)$. The relative decrease in 99 -percentile stress, average strain and average wall pressure, due to presence of thrombus, was also significantly larger for group B (p-value $<0.01,0.03$ and 0.02 ).

Table 4.2: AAA demographical, geometrical and biomechanical characteristics for patients with a relative thrombus volume below (group $A, n=15$ ) and above (group $B, n=15)$ the median relative thrombus volume of $32 \%$.

\begin{tabular}{|c|c|c|c|}
\hline Demographical characteristics & $\begin{array}{c}\text { Group A } \\
(\mathrm{n}=15)\end{array}$ & $\begin{array}{c}\text { Group B } \\
(\mathrm{n}=15)\end{array}$ & p-value \\
\hline Gender (M:F) & $11: 4$ & $14: 1$ & $0.16+$ \\
\hline Age $($ mean $\pm \mathrm{SD}, \mathrm{y})$ & $71 \pm 6$ & $73 \pm 4$ & 0.58 \\
\hline Smoking (never:ever:current) & $2: 9: 4$ & $3: 9: 3$ & $0.84+$ \\
\hline Hypertension & 7 & 10 & $0.07+$ \\
\hline Diabetes Mellitus & 0 & 1 & $0.25+$ \\
\hline Use of statins & 9 & 4 & $0.12+$ \\
\hline \multicolumn{4}{|l|}{ Geometrical characteristics (mean \pm SD) } \\
\hline Relative thrombus volume (\%)\# & $23 \pm 7$ & $54 \pm 15$ & - \\
\hline Maximum diameter (mm) & $51 \pm 4$ & $50 \pm 4$ & 0.67 \\
\hline Lumen tortuosity (-) & $1.4 \pm 0.2$ & $1.4 \pm 0.3$ & 0.57 \\
\hline AAA tortuosity (-) & $1.4 \pm 0.2$ & $1.5 \pm 0.3$ & 0.83 \\
\hline AAA volume (ml) & $132 \pm 40$ & $135 \pm 35$ & 0.82 \\
\hline Lumen volume $(\mathrm{ml}) *$ & $90 \pm 29$ & $58 \pm 16$ & $<0.01$ \\
\hline Thrombus volume $(\mathrm{ml}) *$ & $15 \pm 9$ & $51 \pm 28$ & $<0.01$ \\
\hline Max thrombus thickness $(\mathrm{mm}) *$ & $9 \pm 4$ & $18 \pm 5$ & $<0.01$ \\
\hline Growth rate (median (quartiles), $\mathrm{mm} / \mathrm{y}$ )* & $0(0-1.2)$ & $3(1-6)$ & $<0.01^{*}$ \\
\hline \multicolumn{4}{|l|}{ Biomechanical characteristics (mean \pm SD) } \\
\hline 99-p. stress, no thrombus (kPa) & $465 \pm 89$ & $490 \pm 75$ & 0.45 \\
\hline 99-p. stress, thrombus $(\mathrm{kPa}) *$ & $271 \pm 60$ & $203 \pm 26$ & $<0.01$ \\
\hline 99 -p. stress reduction $(\%) *$ & $43 \pm 8$ & $56 \pm 6$ & $<0.01$ \\
\hline Average strain reduction $(\%) *$ & $1.8 \pm 0.5$ & $3.4 \pm 1.1$ & 0.03 \\
\hline Wall pressure reduction $(\%) *$ & $19 \pm 5$ & $36 \pm 12$ & 0.02 \\
\hline
\end{tabular}




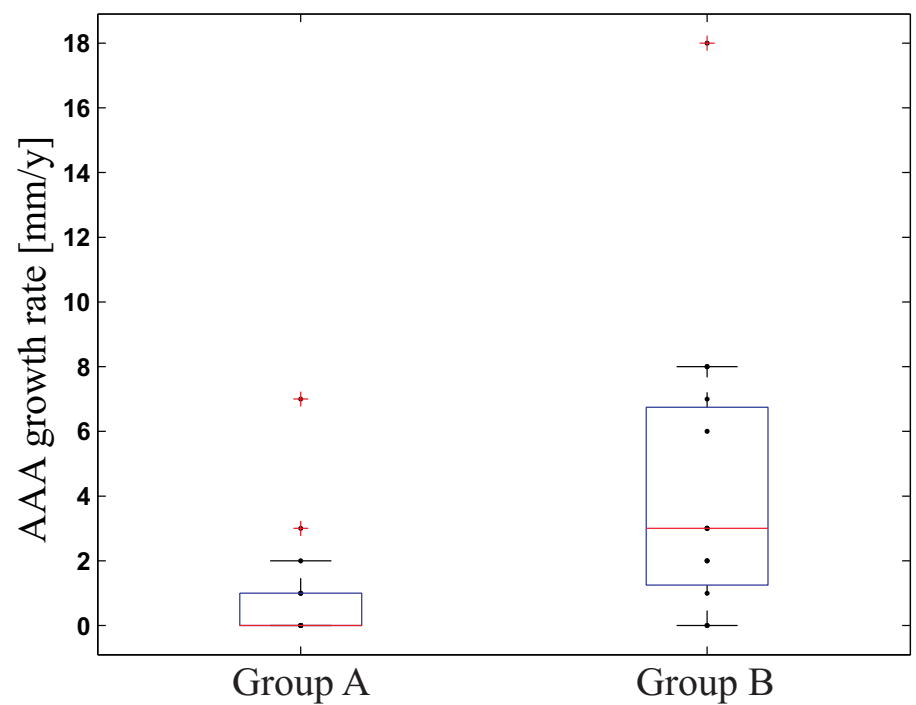

Figure 4.4: Box-and-Whisker plot for the AAA growth rates (mm/y) of groups $A$ and $B$ (Kruskal-Wallis test, $p$-value $<0.01$ ).

\section{Wall Stress Distributions}

Figure 4.6 displays the maximum principal stress in a longitudinal cut-through of an AAA with a relative small thrombus ( $23 \%$ of the total AAA volume). The outer wall stress distribution is not markedly influenced by the presence of the small layer of thrombus (maximum thickness $6 \mathrm{~mm}$ ). However, the inner surface of the wall shows a strong reduction in wall stress. As the nodes on the inner wall surface are connected to both a wall and thrombus element, the displayed wall stress is an average of both. Therefore, the inner wall surface is excluded from the determination of the 99-percentile wall stress, in all stress analyses. The 99-percentile wall stress for this AAA reduced from $321 \mathrm{kPa}$ to $231 \mathrm{kPa}(-28 \%)$ in the presence of thrombus.

Figure 4.7 shows the cut-through of an AAA with a large thrombus $(55 \%$ of the total AAA volume). The thrombus locally reduces the wall stress on the outer wall at locations of considerable thrombus thickness (see arrows, maximum thickness $18 \mathrm{~mm}$ ). The 99-percentile wall stress reduced from $355 \mathrm{kPa}$ to $225 \mathrm{kPa}(-37 \%)$ due to the thrombus.

The main direction of the maximum principal stress is circumferential for all AAA's. In longitudinal direction, stresses are about half the circumferential stress. The stress in radial direction experienced by the inner surface of the AAA wall equals the pressure and is about one order lower than the circumferential stress. 


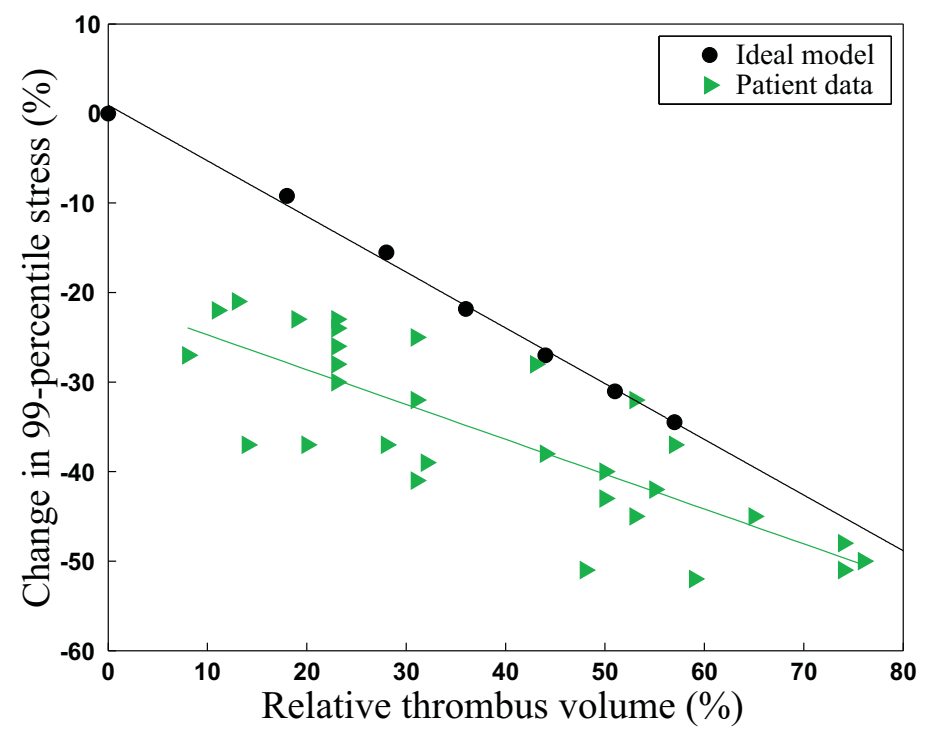

Figure 4.5: The change in wall stress due to the thrombus and the relative thrombus volume for all patient-specific AAA models and for the idealized axisymmetric AAA model (thrombus shear modulus of $40 \mathrm{kPa}$ ).

\section{9-Percentile Wall Stress}

Figure 4.5 shows the decrease in wall stress due to the thrombus for the patients as well as for the idealized model, both with a thrombus shear modulus of $40 \mathrm{kPa}$. The decrease in wall stress due to the thrombus in the patient-specific models is generally underestimated by the results of the ideal model, although the differences are smaller at higher relative thrombus volumes. Both the ideal and patient data show a linear relation with the relative thrombus volume, although for the patient data, the Yintercept is below $0 \%$. Additionally, a considerable variation in the patient-specific results is observed.

\subsection{Discussion}

The risk of an AAA is currently estimated by the maximum diameter. This estimation fails in some cases to accurately predict the rupture risk, but improvement has been shown by using AAA wall stress instead of maximum diameter as risk criterion (Fillinger et al., 2002; Truijers et al., 2007; Venkatasubramaniam et al., 2004). Intraluminal thrombus was previously linked to AAA progression and risk (Hans et al., 2005; Satta et al., 1996; Wolf et al., 1994). In the current study, the effect of thrombus on AAA wall stress was evaluated using finite element analyses with patient-specific and idealized AAA models. The results from the idealized AAA model show that the decrease in wall stress due to the presence of thrombus strongly 
depends on the shear modulus and volume of the thrombus. The relation between the decrease in wall stress and the shear modulus was found to be nonlinear; the decrease in wall stress is less influenced by a change in shear modulus at higher shear moduli. For a relative thrombus volume of $30 \%$, the reduction in wall stress was $22 \%$ with a thrombus shear modulus of $40 \mathrm{kPa}$, whereas it was $8 \%$ with a shear modulus of $10 \mathrm{kPa}$ and only $4 \%$ with a shear modulus of $4 \mathrm{kPa}$. The recently reported shear moduli based on shear and compression experiments were found to be in the order of $10 \mathrm{kPa}$ (Hinnen et al., 2007), or even lower (Ashton et al., 2009; van Dam et al., 2008). However, the same studies also reported considerable variations in shear modulus (see table 4.1), indicating that between and within different thrombi, the shear modulus may vary markedly. Considering that the resulting wall stress is strongly influenced by the shear modulus, the choice of using an average shear modulus for each thrombus in AAA wall stress analyses may therefore not be valid. Dynamic imaging of AAA's, like gated CTA or MRI, may be employed to give more insight in local thrombus material parameters and the effect of thrombus on the wall stress on a patient-specific basis.

Both ideal and patient-specific AAA models show a linear relation between the relative thrombus volume and the effect on the wall stress, although the average model of the patient data shows a less steep relation and a Y-intercept below $0 \%$. This might be explained by the assumption of a minimum layer of $2 \mathrm{~mm}$ thrombus throughout each AAA. This way of modeling was chosen to obtain a robust segmentation and meshing procedure that eventually can be used in a clinical setting. Although this choice was also made for the idealized models and in this case the Y-intercept was $0 \%$, it may be that the effect is different for non-idealized AAA geometries. For larger thrombi, the results of the idealized and patient-specific models are closer together. The effect of the $2 \mathrm{~mm}$ layer of thrombus may therefore be smaller for patient-specific models with larger thrombi. Without this layer the patient-specific results might approach the results from the idealized model. Nevertheless, a considerable variation in effect was found in the patient data. Likely, not only the amount of thrombus influences the wall stress, but also the geometry of the AAA and the thrombus. Therefore, to estimate the effect of thrombus on the patient-specific wall stress, idealized models are inadequate and patient-specific models are required.

The 30 AAA's were split in two equally sized groups, according to their relative thrombus volume $(<$ and $>$ median). The groups matched for age, gender, maximum AAA diameter, total AAA volume and tortuosities. The diameter growth rate was significantly higher in the group with large thrombus volume (figure 4.4, p-value $<0.01)$. This supports findings from previous research on the relation between thrombus and AAA growth (Wolf et al., 1994).

Although no difference was found in wall stress between both groups when thrombus was not included in the analyses, the wall stress was significantly lower for the large thrombus group when thrombus was incorporated (p-value $<0.01$ ). This suggests that diameter growth of AAA's is not instigated by the stress in the wall. Vorp et al. (2001) previously suggested that hypoxia of the AAA wall due to the presence of thrombus may lead to increased inflammation and wall weakening. In research of the same group, thrombus thickness was identified as one of the parameters that lowers 
AAA wall strength (vande Geest et al., 2006c). If this wall weakening is the basis for AAA growth, this may explain the increased growth for AAA's with a relative large thrombus. Future research may evaluate the relation between the amount of thrombus, wall weakening and the growth in diameter of AAA's.

The computed pressure drop over the thrombus for the patient-specific AAA models was fairly large in both groups (19\% and $36 \%$ on average, table 4.2$)$. This pressure drop is in contrast to earlier measurements (Schurink et al., 2000; Thubrikar et al., 2003). In these studies, it was concluded that thrombus leads to a very small pressure drop over the thrombus in AAA's. In this and previous AAA wall stress studies, thrombus has been modeled as a solid structure. It was previously postulated that thrombus has a poro-elastic character (Adolph et al., 1997; Boschetti et al., 2007). Modeling thrombus as a porous material may be more appropriate. In that case, the pressure can be transported through the pores of the material and a smaller pressure drop over the thrombus may be expected. The effect that a poro-elastic material model for the thrombus has on the wall stress cannot be predicted on forehand. Before the effect of poro-elasticity of thrombus can be determined in AAA wall stress computations, parameters like porosity and viscous permeability first need to be determined by experimental testing.

Some limitations of this study need to be mentioned. A constant wall thickness was applied to the AAA wall, although it is known that wall thickness may vary strongly within and between patients (Hellenthal et al., 2008). This may strongly affect the resulting wall stress, but currently, no non-invasive techniques are available to measure local wall thickness. The presence of calcifications was also neglected. Calcifications will have a significant effect on the local wall stress (Li et al., 2008; Speelman et al., 2007), but the implementation of calcifications is debatable, as the correct material properties and interaction between AAA wall and calcifications remain unknown.

Wall stress simulations using the patient-specific AAA models with thrombus shear moduli below $40 \mathrm{kPa}$ resulted in numerical instabilities, due to the complex geometries of both the thrombus and the AAA. Large displacements in the thrombus may occur due to the low shear modulus, which might be resolved using remeshing algorithms during the simulations. This complicates the computational procedure drastically and is currently not implemented in the used software. The results from the idealized model indicates that, for lower shear moduli, the effect of thrombus decreases. However, as a considerable variation exists between and within thrombi, it is important to first focus on determining individual material behavior of thrombus using non-invasive dynamic imaging.

\subsection{Conclusions}

A larger thrombus was not only associated with lower wall stress, but also with a higher AAA growth rate. Therefore, wall weakening under the influence of thrombus, and not wall stress, may be the underlying cause of an increased AAA growth rate (vande Geest et al., 2006c; Vorp et al., 2001). Additional research is required to evaluate the relation between thrombus, AAA wall strength and AAA growth rate. 


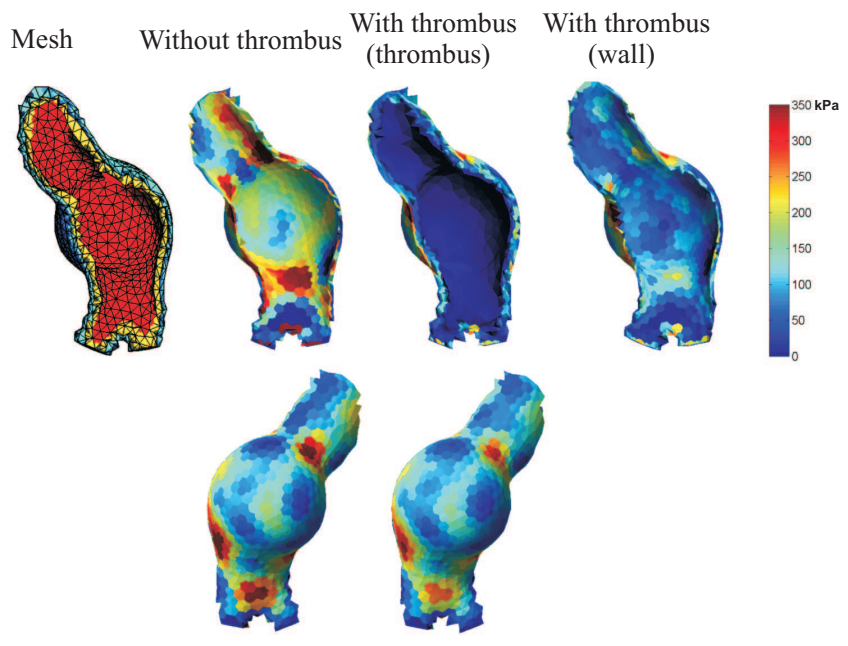

Figure 4.6: Wall stress distributions for an $A A A$ with a $23 \%$ relative thrombus volume. Anterior (top row) and posterior (bottom row) views of the cut-through mesh (lumen boundary in red, thrombus in yellow, wall in blue) and the wall stress without thrombus and with thrombus.

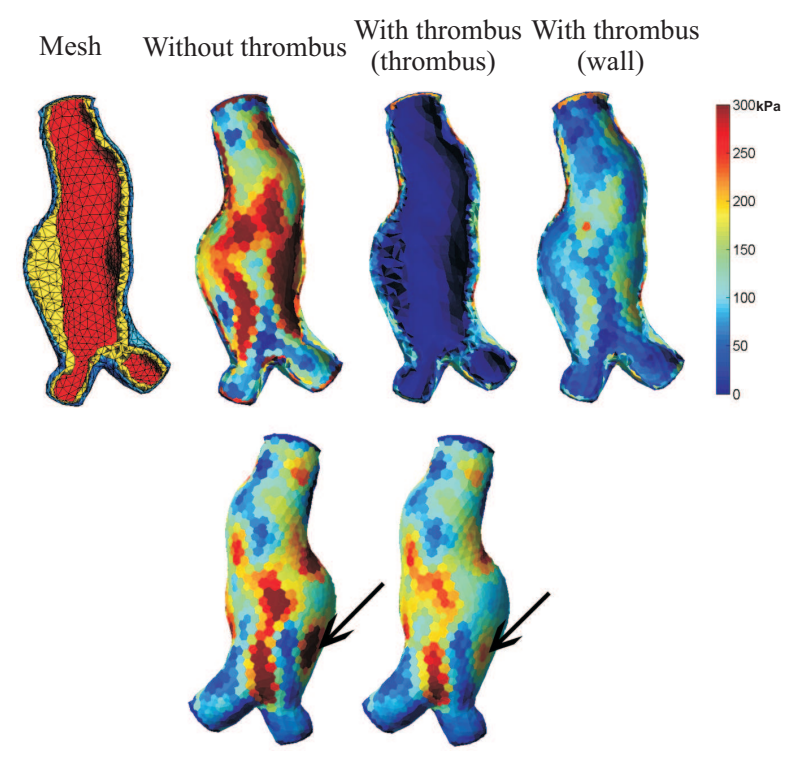

Figure 4.7: Wall stress distributions for an $A A A$ with a $55 \%$ relative thrombus volume. Anterior (top row) and posterior (bottom row) views of the cut-through mesh (lumen boundary in red, thrombus in yellow, wall in blue) and the wall stress without thrombus and with thrombus. The black arrows indicate locations of changed wall stress due to the presence of thrombus. 
$46 \quad$ Chapter 4 


\section{Chapter 5}

\section{Effects of Calcifications in Patient-Specific AAA Wall Stress Analyses}

The objective of this study was to evaluate the influence of calcifications on wall stress using patient-specific AAA finite element models. In addition, the relation between the effect of calcifications and the amount of calcification present in the AAA wall was evaluated.

Finite element models were created of six AAA's and the areas of calcification were defined node-wise in the mesh of the model. Wall stress was computed without and with calcifications using different material properties. Peak stresses were subsequently compared to the calcification index (CI).

Stresses were increased at local calcification sites, leading to a peak stress increase up to $22 \%$. A weak correlation was found between the CI and the increase in peak stress. Additionally, the results showed a marked influence of the calcification elastic modulus on computed stresses.

The results indicate that the location and shape of the calcified regions -not only the relative amount- are considerations that influence the effect on AAA wall stress. Therefore, to estimate the effect of the calcifications, patient-specific modeling is required. The dependency of effect on the wall stress on the calcification elastic modulus points out the importance of determination of the material properties of calcified AAA wall.

This chapter is based on: L. Speelman, A. Bohra, E. M. Bosboom, G. W. Schurink, F. N. van de Vosse, M. S. Makaroun and D.A. Vorp, Effects of wall calcifications in patient-specific wall stress analyses of abdominal aortic aneurysms, J. Biomech. Eng. 2007, 129(1):105-9. 


\subsection{Introduction}

An abdominal aortic aneurysm (AAA) is a permanent local dilation of the aorta in the abdominal cavity of more than $50 \%$ the original diameter. Although the cause of AAA is not fully understood, the dilation is most likely a result of inflammation and degeneration of the media of the aortic wall (Daugherty and Cassis, 2002).

Of the patients with an AAA, $75 \%$ remain symptom free until rupture of the AAA occurs, which is fatal in most cases. Annually, 15,000 to 20,000 deaths occur in the USA due to AAA rupture (Bush et al., 2003). The annual rupture rate exponentially increases with the diameter of the aortic aneurysm from $1 \%$ when they are smaller than $5.4 \mathrm{~cm}$ to $32.5 \%$ when they are larger than $7 \mathrm{~cm}$ (Gorham et al., 2004). Elective repair procedures, however, are also not without risks and are associated with 30-day mortality rates up to 5\% (Greenhalgh et al., 2004).

Because rupture of an AAA is fatal in most cases and an elective repair procedure is not without risks, it is important to assess the rupture risk. Rupture risk of an AAA is currently predicted only by maximum diameter of the aneurysm, although this criterion is not always sufficient. Smaller AAA's rupture occasionally and AAA's with diameters well over $5 \mathrm{~cm}$ do not always rupture, so a repair procedure may expose the patient to unnecessary risks (Choke et al., 2005).

An increasing number of studies have focused on relating rupture risk to AAA geometry, morphology and resultant wall stress rather than the currently used diameter (Hall et al., 2000; Mower et al., 1997; Raghavan et al., 2000). In these studies, the underlying principle is that rupture of the AAA wall occurs when the stress acting on the wall exceeds some critical value. Recently, methodologies have been developed to estimate peak wall stress with patient-specific finite element models of AAA's (Raghavan et al., 2000; Wang et al., 2002). Using such methodology, peak wall stress appears to assess rupture risk more accurately than AAA diameter or other previously proposed clinical indices (Fillinger et al., 2002, 2003). More and more studies have been done to increase the accuracy of these patient-specific models by including geometrical and morphological details. Thubrikar et al. (2001) incorporated wall thickness variation in AAA models with thicknesses ranging from 1.31 to $1.58 \mathrm{~mm}$. This thickness variation caused stresses to vary up to $21 \%$, which is consistent with what would be expected based on the law of Laplace. Also, to improve the accuracy, intraluminal thrombus has been incorporated in the models in several studies (di Martino et al., 1998; Mower et al., 1997; Wang et al., 2002). Di Martino et al. (1998) concluded from finite element simulations that thrombus significantly reduces the effect of the pressure load on the aneurysm wall. Wang et al. (2002) found that the presence of intraluminal thrombus influenced not only the peak wall stress, but also the stress distribution on the AAA wall. The effect of calcifications on wall stress, however, has not yet been studied. As calcifications generally have a higher stiffness than the surrounding wall, it is hypothesized that calcifications have a significant influence on the stress distribution acting on the AAA wall. Therefore, the purpose of this study was to evaluate the influence of wall calcifications on AAA wall stress. 


\subsection{Methods}

\subsubsection{Calcifications}

CTA scans of six AAA's, which were used previously to create 3D finite element models (Raghavan et al., 2000; Wang et al., 2002), were examined again to take calcifications of the AAA wall into account. As before, the outer wall of the infrarenal aorta was identified from the CTA scans and smoothed with a surface-smoothing algorithm. Calcifications have a high attenuation coefficient and are therefore clearly visible in CTA scans as high-intensity regions (figure 5.1). A custom computer program was written to semi-automatically process each CTA slice from the renal arteries to the bifurcation in the abdominal aorta.

To perform this segmentation, a region of interest was first drawn around the aorta to distinguish it from the spine and the ribs, as bone has a similar attenuation coefficient as the wall calcifications (see the dashed contour in figure 5.1a). The mean

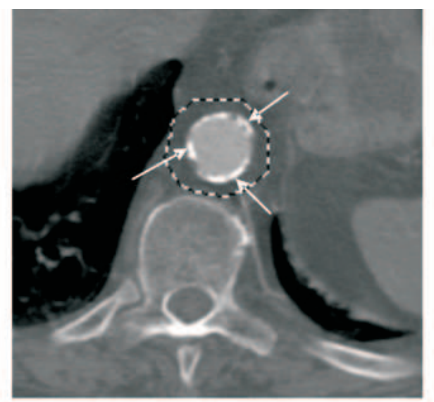

(a)

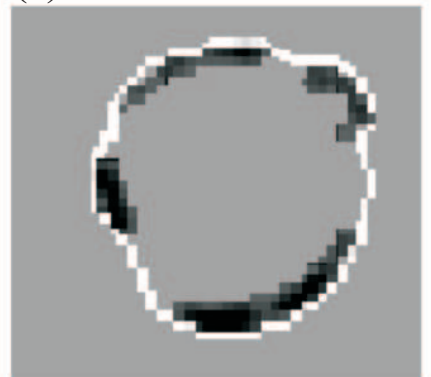

(c)

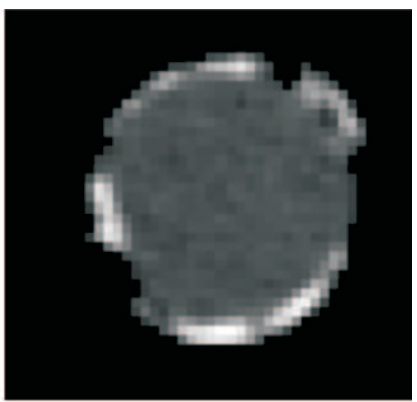

(b)

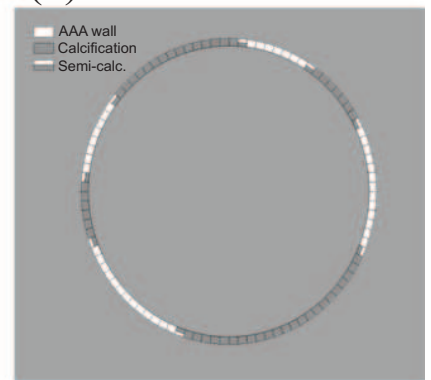

(d)

Figure 5.1: (a) CTA slice of an AAA with region of interest indicated by dashed contour and wall calcifications indicated with white arrows. (b) The aortic contour separated from the surrounding tissue. (c) The detected edge of the aorta (white) and the calcifications (dark). (d) The equivalent element representation with material properties of the AAA wall assigned to the white elements and of the calcified wall to the grey elements. The transition between the wall and calcified regions are represented by the double layered elements. 
and standard deviation (SD) of the intensity of the blood was determined, which is dependent on the amount of contrast agent present in the blood at the time of the CTA scan. An empirically determined threshold of the mean minus four times the SD of the lumen intensity was applied to the region of interest to separate the lumen and the calcifications from the surrounding tissue, which results in figure 5.1b. After this, the calcifications were identified as pixels with an intensity above the, also empirically determined, threshold of the mean plus four times the SD of the lumen intensity (see figure 5.1c). The coordinates of the detected calcified pixels were transformed to polar coordinates with the center of mass of the aortic lumen defined as the origin. Each calcification was then characterized by the spatial coordinates of the middle of the calcification and the area of the wall contour covered by the calcification.

The areas of calcification were then defined in terms of nodes in the finite element mesh of the AAA models, using the spatial information of the calcifications (figure 5.1d). The CTA data and the finite element models were visually compared to ensure that the extent and locations of the calcifications were accurately incorporated.

To express the overall degree of calcifications for a single AAA via a single parameter, a Calcification Index (CI) was defined as the percentage of total wall surface area occupied by calcifications.

\subsubsection{Material Properties and Boundary Conditions}

The AAA wall and intraluminal thrombus were modeled with two-parameter, hyperelastic, isotropic and incompressible material models from Raghavan and Vorp (2000) and Wang et al. (2001). The models are described with the following constitutive equations:

$$
\begin{cases}\text { AAA wall : } & \boldsymbol{\sigma}=-p_{h} \boldsymbol{I}+2\left(\alpha+2 \beta\left(I_{B}-3\right)\right) \boldsymbol{B} \\ \text { Thrombus : } & \boldsymbol{\sigma}=-p_{h} \boldsymbol{I}-2\left(c_{1}+2 c_{2}\left(I I_{B}-3\right)\right) \boldsymbol{B}^{-1}\end{cases}
$$

In these equations, $\boldsymbol{\sigma}$ is the Cauchy stress tensor, $p_{h}$ is the hydrostatic pressure, $\boldsymbol{I}$ is the unity tensor and $\boldsymbol{B}$ the left Cauchy-green deformation tensor. $I_{B}$ and $I I_{B}$ are the $1^{\text {st }}$ and $2^{\text {nd }}$ invariant of $\boldsymbol{B}$. The model parameters $\alpha, \beta, c_{1}$ and $c_{2}$ were estimated for each specimen tested. The mean plus or minus the standard error of the mean (SEM) for $\alpha$ and $\beta$ within the abdominal aortic aneurysm patient population was found to be $0.17 \pm 0.2 \mathrm{kPa}$ and $1.9 \pm 0.4 \mathrm{MPa}$, respectively (Raghavan and Vorp, 2000). For $c_{1}$ and $c_{2}$, the mean and SEM was found to be $26 \pm 8 \mathrm{kPa}$ and $26 \pm 8 \mathrm{kPa}$ (Wang et al., 2001).

Fully calcified elements consisted of all calcified nodes and were given a linear elastic, Neo-Hookean material behavior and were assumed to be nearly incompressible (Poisson ratio 0.45 ) and isotropic. To avoid computational errors or convergence problems due to large material transitions, elements formed by a combination of calcified and non-calcified nodes, typically situated at the boundaries of a calcified area, were defined as semi-calcified elements. A mean wall thickness of $1.5 \mathrm{~mm}$ found by ex-vivo measurements was used throughout the whole AAA model (Vorp, 1997). The semi-calcified elements were taken as a bi-layered composite material, 
with the two layers, each assigned a thickness of $0.75 \mathrm{~mm}$ of either aneurysmal tissue or calcifications (figure 5.1d).

To our knowledge, no studies have been done to determine the material properties of calcified abdominal aortic aneurysm tissue. Loree et al. (1994) studied calcified aortic atherosclerotic tissue and found a circumferential tangential modulus of $1.47 \pm 1.28 \mathrm{MPa}$. Because of the large standard deviation and the usage of data of non-AAA tissue, we also investigated the influence of variation in material stiffness of the calcifications. Specifically, simulations were performed on all six AAA models using the mean value of the elastic modulus for the calcifications (i.e. 1.47 MPa) and the mean elastic modulus plus or minus the SD (i.e. 0.19 and $2.75 \mathrm{MPa}$, respectively). The boundary conditions of the simulations were a uniform pressure of $16 \mathrm{kPa}$, applied to the inner surface of the models, and fixation in every direction of the upper and lower ring of elements.

\subsubsection{Finite Element Analysis}

The finite element solver used in the study was Abaqus/Standard (v6.2, Abaqus Inc., Pawtucket, RI). The wall of the AAA and the calcified regions were each modeled with linear quadrilateral shell elements (Abaqus element S4R), which employ 5 integration points through the thickness of the element to interpolate the stresses. The composite elements, however, consisted of 2 layers, each with 3 integration points.

Thrombus was modeled with linear hexahedron-shaped elements with 8 nodes (Abaqus element C3D8H). A 1:1 coupling of wall and thrombus elements prohibited any movement of the interfacial elements with respect to each other. The incompressibility of both the AAA wall and the thrombus was handled by a penalty function; i.e., by adding a compression function to the constitutive equations of the finite element model. This method is commonly used in finite element modeling to handle the incompressibility condition and details are thus beyond the scope of this article. As this is a standard feature of the software used, the interested reader is referred to the Abaqus user manual for further information.

After computing the stresses on the wall with and without the calcifications, the relation between peak stress increase due to the calcifications and the CI was assessed. All stress results were displayed in terms of maximum principal stress. Peak stress was defined for each AAA as the highest maximum principal stress found in the wall of that model.

\subsection{Results}

Visual comparison of the CTA data and the locations of the calcifications in the $3 \mathrm{D}$ reconstructed AAA models (see figure 5.2a) revealed that our procedures were suitable. 


\subsubsection{AAA Wall Stress}

The stress distributions from the finite element simulations using calcifications with an elastic modulus of $0.19 \mathrm{MPa}$ were indistinguishable from the stress distributions without calcifications (figure 5.2b). However, the wall stress distributions of all six models using calcifications with an elastic moduli of 1.47 and $2.75 \mathrm{MPa}$, were markedly altered (figure 5.2c and 5.2d, respectively).

Note that the stress contours in figure $5.2 \mathrm{~b}$ are smooth and caused by the geometry of the AAA's alone, whereas the contours in figures $5.2 \mathrm{c}$ and $5.2 \mathrm{~d}$ are less smooth due to the calcifications incorporated in the models. The calcifications in general also resulted in markedly elevated peak wall stress (see table 5.1).

\subsubsection{Calcification Index}

The CI for all six models is tabulated in table 5.1, together with peak stress in the AAA models, both with and without calcifications. Three of six models (models 2, 3 and 5) exhibited an increase in peak stress when the calcification elastic modulus was taken as 1.47 MPa, while two models showed no increase (models 1 and 4) and one model (model 6) exhibited a decrease. All six models, using an elastic modulus of $2.75 \mathrm{MPa}$, exhibited an elevated peak stress ranging from $3 \%$ to $22 \%$ increase over the non-calcified counterparts. The Spearman rank order correlation coefficients between the CI and the peak stress increase due to the calcifications were 0.31 and 0.60 for the elastic moduli of 1.47 and 2.75 MPa, respectively.

Table 5.1: Calcification Index (CI) and peak stress in $\mathrm{kPa}$ for all models without and with calcifications with an elastic modulus of 1.47 and $2.75 \mathrm{MPa}$. The percent change in peak stress due to the calcifications compared to the non-calcified model is indicated parenthetically. $R_{s}$ is the Spearman rank order coefficient between the CI and the percentage of peak stress increase due to the calcifications.

\begin{tabular}{lcccc}
\hline \hline \multicolumn{2}{l}{ Peak stress (kPa) } & No Calcifications & \multicolumn{2}{c}{ Calcification Elastic Modulus } \\
\hline Model & CI & - & $1.47 \mathrm{MPa}$ & $2.75 \mathrm{MPa}$ \\
\hline 1 & 0.05 & 342 & $343(+0 \%)$ & $422(+22 \%)$ \\
2 & 0.38 & 310 & $341(+10 \%)$ & $380(+22 \%)$ \\
3 & 0.06 & 262 & $280(+10 \%)$ & $307(+18 \%)$ \\
4 & 0.02 & 383 & $383(+0 \%)$ & $394(+3 \%)$ \\
5 & 0.05 & 471 & $484(+2 \%)$ & $509(+6 \%)$ \\
6 & 0.16 & 510 & $496(-4 \%)$ & $551(+8 \%)$ \\
\hline$R_{s}$ & & & 0.31 & 0.60
\end{tabular}




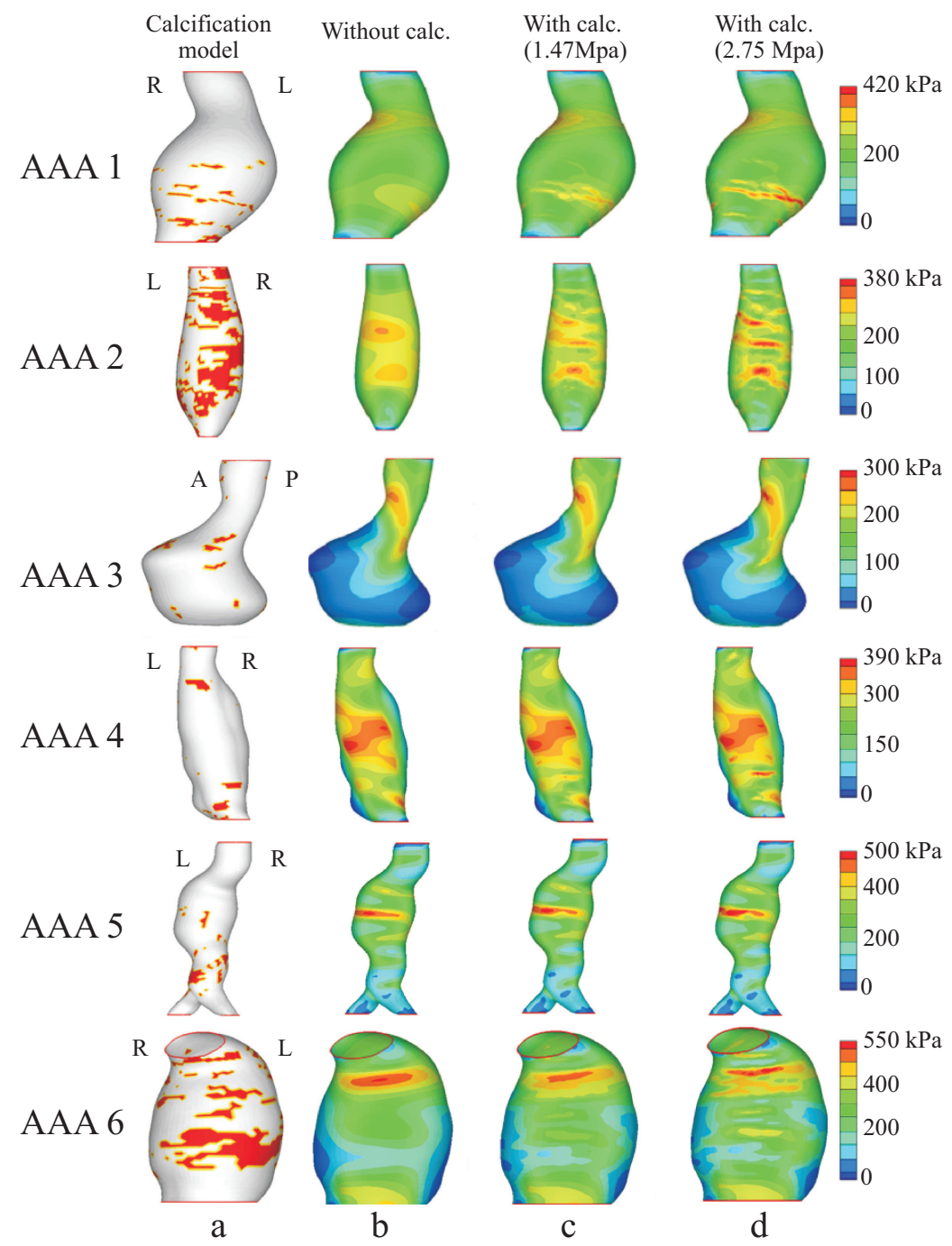

Figure 5.2: The six AAA models studied here, with the calcifications noted in red for fully calcified elements and yellow for semi-calcified elements (a). The in-vivo spatial orientation is indicated with $A$ (anterior), $P$ (posterior) or $L$ (left), $R$ (right). Maximum principal stress distribution (in $\mathrm{MPa}$ ) is shown for the models without calcifications (b), with calcifications with an elastic modulus of $1.47 \mathrm{MPa}(c)$, and with calcifications with an elastic modulus of $2.75 \mathrm{MPa}(d)$. The 'end effects' (i.e. artificial stresses induced by the end fixations) were avoided by excluding stresses in the regions defined by the upper and lower $10 \%$ of the model. 


\subsection{Discussion}

Previous wall stress simulations have suggested that peak stress gives a better rupture risk estimate than maximum transverse diameter of the AAA (Fillinger et al., 2003). In this study, the effect of AAA wall calcifications on the wall stress, as computed with patient-specific finite element simulations was studied. The incorporation of calcifications markedly influenced the AAA wall stress. In general, the calcifications caused local increased stresses, the extent of which depends on the elastic modulus of the calcifications.

To study whether the global effect of all calcification of a particular AAA can be determined without the use of finite element analysis, the relationship between the percentage of calcification in the AAA wall (CI) and the peak AAA wall stress was examined. However, only a minor correlation was found, suggesting that finite element models are essential in determining the effect of the wall calcifications on a patient-specific basis. That is, the location and shape of the calcified regions -not just the relative amount- are considerations that more greatly influence the effect on the wall stress. For example, if calcifications are located in areas predicted to have high stress even without calcifications, the effect of the calcifications on wall stress is pronounced, even if its stiffness is only slightly higher than that of the AAA wall. On the other hand, if calcifications are present in lower stress areas, only a significantly stiff calcification will substantially increase the wall stress. This may explain variable effects observed in figure 5.2. For example, model 1 contains no calcifications in high stress areas, and subsequently no significant stress increase is noted when the calcifications have an elastic modulus of $1.47 \mathrm{MPa}$. However, model 2 has calcified areas at locations of high stress and a marked increased in wall stress is noted. For a calcification elastic modulus of 2.75 MPa, both models have an elevated peak stress. In this study, several assumptions were made that should be discussed. First, any initial stress that may exist in the AAA wall was neglected. Previous studies showed that initial stresses strongly influence the resulting wall stresses (Lu et al., 2007; Speelman et al., 2009a). However, no methods to correct for initial stress were available at the time of this research. Also residual stresses were neglected. Based on previous research it may be assumed that these are small in comparison to the stresses resulting from the intra-aneurysm blood pressure (Alastrué et al., 2007). Ignoring the effect of initial stress and residual stress may have influenced the results, although it may expected that the effect is small, as the situation with and without calcifications is mutually compared. The effect would likely be comparable in both situations.

The boundary conditions did not include contact of the AAA with surrounding organs and the vertebral column, which may have influence on the results (Gasbarro et al., 2005). As the aim of this study was to evaluate the influence of calcifications on AAA stress computations by comparing the results with and without calcifications, and since all these assumptions are similar in both models, we believe that our conclusions remain valid.

The material properties of non-aneurysmal calcifications were used in the simulations, which may be significantly different from those from aneurysmal calcifications. Therefore, the elastic modulus of the calcifications was varied in this study, ranging 
from 0.19 to $2.75 \mathrm{MPa}$. We found a large dependency of AAA peak wall stress on the stiffness of the calcifications, which underlines the importance of determination of the material properties of calcified AAA wall. Holzapfel et al. (2004) recently published the results of mechanical testing of calcified iliac arteries. He found a Young's modulus of $12.6 \pm 4.7 \mathrm{MPa}$, which is significantly higher than the elastic modulus found by Loree et al. (1994) and used in this article. Based on our observations, it is likely that if the higher modulus values of Holzapfel et al. (2004) were used, the influence of calcifications on wall stress would be even more pronounced.

Another potential limitation is the manner by which the calcified areas were modeled in this study. It was assumed that the calcifications have a rough surface, based on the observation that they have a lower intensity towards their edges in the CTA images. This may imply an ingrowth of wall material in the calcifications. To account for this and to prevent artificial stresses due to high material transitions without significantly increasing simulation times, the boundaries of calcified areas were modeled as bilayer shell elements. A more accurate approach might be to incorporate a smoother gradation of calcification within the AAA wall, based on the greyvalues in the CTA scan, and future work should explore this. Another possibility is that, due to the varying blood pressure, calcifications become separated from the AAA wall and thus exist in local "holes" within the tissue. Previously, research from our group on calcifications using $\mu \mathrm{CTA}$ revealed that what appears as a solid, uniform calcification in normal resolution CTA images, may in fact be a complex arrangement of smaller calcified areas (de Putter et al., 2006b). This means that it is of little use to try to accurately model the shape and the material interface of the calcification, because the normal CTA image resolution is too low to capture these details. Future research should therefore focus on accurately establishing the material properties of calcified regions, and on experimentally assessing the rupture potential of the calcifications, the material interface and the surrounding non-calcified tissue.

A mesh independency study was previously performed by Wang et al. (2002) in an earlier study where the same AAA models were used. As the aim of the current study was to explore the importance of including calcifications in patient-specific AAA wall stress simulations, and not to optimize the methods, no further mesh refinement study was performed. However, any future incorporation of calcifications into patient-specific finite element models for the purposes of predicting AAA rupture clinically will require individualized mesh refinement in order to optimize predictive accuracy.

Most biomechanical considerations of AAA's have focused on computing stresses acting on the aneurysm wall, and not on its failing strength. This is, however, an equally important part in rupture risk prediction, since rupture occurs when the stresses exceed the strength of the wall. Sherebrin et al. (1987) studied the extensibility changes in calcified aortic wall and concluded that calcifications immobilize a a part of the wall, which may cause a lower breaking strength and strain of the tissue. A combination of a weaker wall and a higher stress, both caused by the calcifications, results in a drastically elevated rupture risk, even higher than predicted by wall stress analysis alone. 


\subsection{Conclusions}

In summary, AAA wall calcifications show a marked influence on the stress acting on the AAA wall (see figure 5.2). This study also proved that the effect of calcifications cannot be determined directly from the CTA scans by determining the amount of calcifications present (see table 5.1). As the location and shape of the calcifications apparently also influence the effect on peak wall stress, patient-specific finite element analysis is essential in the assessment of AAA wall stress with calcifications. This study also showed that the wall stress was strongly dependent on the stiffness of the calcifications, which underlines the importance of determining the material properties of calcified AAA wall before calcifications are incorporated in future AAA wall stress analyses. 


\section{Chapter 6}

\section{The Influence of Wall Stress on AAA Growth and Biomarkers}

With increasing stress in the wall of an abdominal aortic aneurysm (AAA), more damage may occur in the wall, possibly leading to progression of the aneurysm and reflection in up- or downregulation of specific circulating biomarkers. This study investigated the relation between AAA wall stress, AAA growth rate and biomarker concentrations of MMP-9, TIMP-1, CRP and $\alpha 1-\mathrm{AT}$.

Thirty-seven patients (maximum AAA diameter 41-55 mm) with at least two CTA scans were prospectively included. Diameter growth rate in $\mathrm{mm} /$ year was determined between two consecutive CTA scans. AAA wall stress was computed by finite element analysis, based on the first CTA scan only $(n=69)$. Biomarker concentrations were determined in 18 patients. The AAA's were divided in three equally sized groups, based on the stress relative to their diameter (relative low, medium and high stress). Growth rate and biomarker concentrations were compared between the groups. Correlation coefficients were computed between absolute wall stress, AAA growth and biomarker concentrations.

A higher relative wall stress was associated with a higher AAA growth rate. Growth rate was also significantly related to MMP-9 plasma concentration $(\mathrm{r}=0.32)$. The concentrations of MMP-9 and CRP showed a positive trend with the relative wall stress, but no significant relations between wall stress and any of the biomarkers could be identified. Future research is required to verify the relation between wall stress, biomarker concentrations and AAA growth rate.

This chapter is based on: L. Speelman, F. A. Hellenthal, B. Pulinx, E. M. H. Bosboom, F. N. van de Vosse, M. J. Jacobs, M. Breeuwer, M. R. van Sambeek, W. K. W. H. Wodzig and G. W. Schurink, The Influence of Wall Stress on AAA Growth and Biomarkers, submitted to Eur. J. Vasc. Endovasc. Surg. 2009. 


\subsection{Introduction}

Incidence of abdominal aortic aneurysm (AAA) is increasing due to general aging of the population and an increase in the amount of screening programs (Lederle et al., 1997; van der Vliet and Boll, 1997). If left untreated, an AAA will increase in size until rupture of the aortic wall occurs, causing a life-threatening hemorrhage. Growth rate of an AAA is generally defined as the change in maximum aortic diameter over a certain time period. Previous studies indicate that AAA growth rate increases with the diameter of the AAA (Stonebridge et al., 1996; Vega de Céniga et al., 2006). However, this growth rate is not identical for all AAA's, as some AAA's remain stable for a considerable period of time, while others show a strong increase in diameter over a short period. Also, some AAA's tend to grow discontinuously, with alternating periods of growth and non-growth (Kurvers et al., 2004; Vega de Céniga et al., 2008). Recently, multiple studies have focused on patient-specific AAA wall stress analyses (Fillinger et al., 2002; Raghavan et al., 2005; Speelman et al., 2008; Truijers et al., 2007; Venkatasubramaniam et al., 2004). Peak wall stress was found to be significantly higher for patients with symptomatic or ruptured AAA's than for asymptomatic aneurysms (Fillinger et al., 2002; Truijers et al., 2007). However, in these studies, the relation between wall stress and AAA growth rate was not investigated. With increasing wall stress, more damage may occur in the AAA wall, leading to degeneration of the wall and expansion of the aneurysm. Wall stress may thus have a prominent role in aneurysm growth and computing AAA wall stress may lead to a predictive model for AAA growth rate. Circulating biomarkers are believed to reflect inflammation and degeneration in the AAA wall (Golledge et al., 2008). Matrix metalloproteinase-9 (MMP-9) is involved in the breakdown of the extracellular matrix and a higher plasma MMP-9 concentration was associated with AAA presence (Golledge et al., 2008). Additionally, MMP-9 significantly correlated with AAA growth rate (Lindholt et al., 2000). The activity of MMP's is, amongst others, controlled by tissue inhibitor of the metalloproteinases-1 (TIMP-1). Although plasma concentrations of TIMP-1 were found to be significantly higher in AAA's than in healthy controls (Nakamura et al., 2000), TIMP-1 levels were found to be lower in AAA wall tissue compared to healthy aortic tissue (Wilson et al., 2005). Creactive protein $(\mathrm{CRP})$ is a non-specific acute phase protein that is rapidly expressed in inflammation and has been previously linked to AAA size (Vainas et al., 2003). Recently, alpha 1-antitrypsin $(\alpha 1-\mathrm{AT})$, an inhibitor of serine proteases such as trypsin and leukocyte elastase, was correlated with AAA growth (Vega de Céniga et al., 2009).

Summarizing, AAA's with a relative high wall stress may experience more damage to the AAA wall, possibly leading to faster expansion of the aneurysm. The amount of AAA wall damage may be reflected by up- or downregulation of specific circulating biomarkers. In this study, the relation between AAA wall stress, AAA growth rate and biomarker concentrations was evaluated. First, the relation between maximum diameter and wall stress will be determined, and AAA's with relative high, medium and low wall stress relative to their diameter, will be identified. Prospective AAA diameter growth rate will be compared for each of the wall stress groups and the 
concentrations of MMP-9, TIMP-1, highly sensitive measured CRP (hs-CRP), and $\alpha 1-\mathrm{AT}$ will be studied in relation to the computed wall stress and the prospective AAA growth rate.

\subsection{Methods}

Thirty-seven patients with an asymptomatic AAA (initial maximum diameter 40$55 \mathrm{~mm}$ ) from the Catharina Hospital Eindhoven (the Netherlands, n=19) and the University Medical Center Maastricht (the Netherlands, $\mathrm{n}=18$ ), were prospectively included in the study. CTA scans were made with a 4-month interval until either the patient was eligible for surgery or the maximum of 4 consecutive CTA scans was reached. A standard contrast enhanced hospital protocol for AAA's was used (in-plane resolution $512 \times 512$ pixels, field-of-view $30-35 \mathrm{~cm}^{2}$, reconstructed slice thickness 1-2 $\mathrm{mm}$ ). The patients were included for a 1-year follow-up with CTA, thereafter, regular ultrasound surveillance was continued. Blood pressures were measured within 30 minutes after the CTA scan. Growth in AAA diameter was determined as the change in maximum anterior-posterior diameter between two consecutive CTA measurements and was converted to $\mathrm{mm} /$ year.

Venous blood was drawn via an antecubital vein puncture and collected in SST (serum) and EDTA (plasma) buffered vacutainersß). Exactly thirty minutes after collection, the blood was centrifuged $\left(15\right.$ minutes, $3000 \mathrm{~g}, 4^{\circ} \mathrm{C}$ ) and multiple aliquots were stored at $-80^{\circ} \mathrm{C}$, exactly one hour after collection, until further analyses.

Patient demographical information (age, gender, hypertension, smoking, diabetes mellitus and statin use) was collected at the time of inclusion and updated each visit. Research approval was given by the local Medical Ethics Commissions of the hospitals involved. All patients signed informed consent prior to inclusion in the study.

\subsubsection{Biomarker Analysis}

Biomarker analyses were only performed on the blood samples of the 18 patients from the University Medical Center Maastricht. Plasma levels of MMP-9 and TIMP-1 were determined in duplo by means of commercially available ELISA (GE Healthcare, Upsala, Sweden). The duplo concentration measurements results in two independent values. Whenever these values deviated less than $10 \%$ of each other, the average value was used in the analyses. Otherwise, the measurements were discarded. The serological levels of $\alpha 1$-AT and hs-CRP were routinely determined on the BN Prospec (Dade Behring Inc., Dearfield, USA).

\subsubsection{Wall Stress Analysis}

Automatic segmentation of the AAA's from the contrast enhanced CTA data was done as described previously (Speelman et al., 2008, see chapter 2). No thrombus or calcifications were incorporated in the finite element model, but the relative thrombus volume (Speelman et al., 2009b) and calcification index (Speelman et al., 2007) were determined for each model. The segmentation of the AAA wall was used as input 
to create a finite element mesh. A mesh typically consisted of approximately 30,000 quadratic 15-node tetrahedral elements, and a constant wall thickness of $2 \mathrm{~mm}$ was applied. A mesh refinement study was performed prior to the analyses and showed that the wall stress results changed less than $1 \%$ for an increasing number of elements. The finite element software Sepran (Sepra, Delft, the Netherlands) was used to calculate the AAA wall stresses. The governing equations of mass and momentum were solved and the most distal and proximal planes of the models were constrained in all directions as essential boundary conditions (see Appendix A for the finite element formulation of the mass and momentum equations). The nonlinear material model as proposed by Raghavan and Vorp (2000) was used to model the aortic wall behavior. The patient group averaged systolic blood pressure of $140 \mathrm{mmHg}(18.7 \mathrm{kPa})$ was applied to the inner wall of the finite element model. No cardiac triggering was applied in the CTA imaging protocol, therefore, the AAA geometry as derived from the CTA, was subjected to a time-averaged blood pressure. This results in initial stresses in the AAA wall, which was accounted for using the backward incremental (BI) method (de Putter et al., 2007; Speelman et al., 2009a, see chapter 3). The mean arterial pressure, as measured after the CTA scan, was used for that purpose.

Stresses, strains and displacements were calculated throughout the whole aneurysm model. In each node, maximum principal stress was computed. 99-percentile wall stress in the AAA was defined as the highest stress, after exclusion of $1 \%$ of the nodes in the mesh with the highest stress. 99-percentile wall stress showed to be more reproducible and less sensitive to geometrical variations than peak wall stress (Speelman et al., 2008, see chapter 2).

\subsubsection{Data Analysis}

Based on the law of Laplace it may be expected that AAA wall stress generally increases with diameter. A regression model between maximum AAA diameter and 99-percentile wall stress was therefore determined for all AAA models. The residuals of the regression model were normalized with the standard deviation of the model, and the AAA's were divided over three equally sized groups (relative high, medium and low stress), based on the normalized residuals. Patient demographics, relative thrombus volume (Speelman et al., 2009b), calcification index (Speelman et al., 2007) and prospective AAA growth were compared for each stress group. The log levels of MMP-9, TIMP-1, $\alpha 1$-AT and hs-CRP were compared between the high, medium and low stress groups, using ANOVA. As the biomarkers may influence each other on different levels, partial correlation coefficients were computed for both AAA growth rate and wall stress, in relation with $\log$ levels of the biomarkers. In that case, the strength of the relation between two variables is measured, after adjusting for the relations with other variables included in the evaluation. A p-value lower than 0.05 was considered significant. The data analyses were performed with Statgraphics Centurion XV (StatPoint, Herndon, Virginia, USA). 


\subsection{Results}

A total of 106 CTA scans were made (range 2-4 scans per patient). The median AAA diameter in these scans was $46 \mathrm{~mm}$ (range 41-57 mm). Of each pair of two consecutive CTA scans, the growth rate was determined and only the first scan was used for wall stress analyses, leaving in total 69 stress-growth comparisons in 37 patients (figure 6.1). During the study, four patients reached a diameter of $55 \mathrm{~mm}$ or more and were scheduled for aneurysm repair.

The left image of figure 6.2 shows the relation between maximum AAA diameter and 99-percentile wall stress for the 69 measurements (r 0.47 , p-value $<0.01$ ). The residuals of the regression model were normalized by the standard deviation (SD) of the model and three equally sized groups were formed with relative low, medium and high stress (figure 6.2 right). The thresholds to form the groups were $\pm 0.5 \mathrm{SD}$.

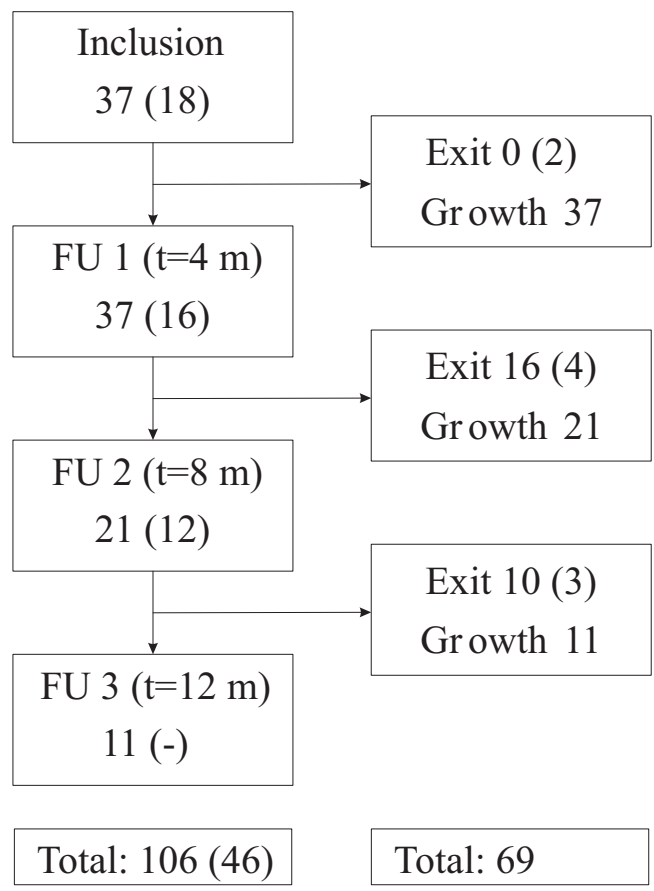

Figure 6.1: The patient flow chart indicating the number of patients with CTA at inclusion and at 4, 8 and 12 month follow-up. In brackets is indicated how many patients were included in the biomarker analysis. The right side indicates the number of patients that were lost between each follow-up and the number of patients used for growth analysis. 

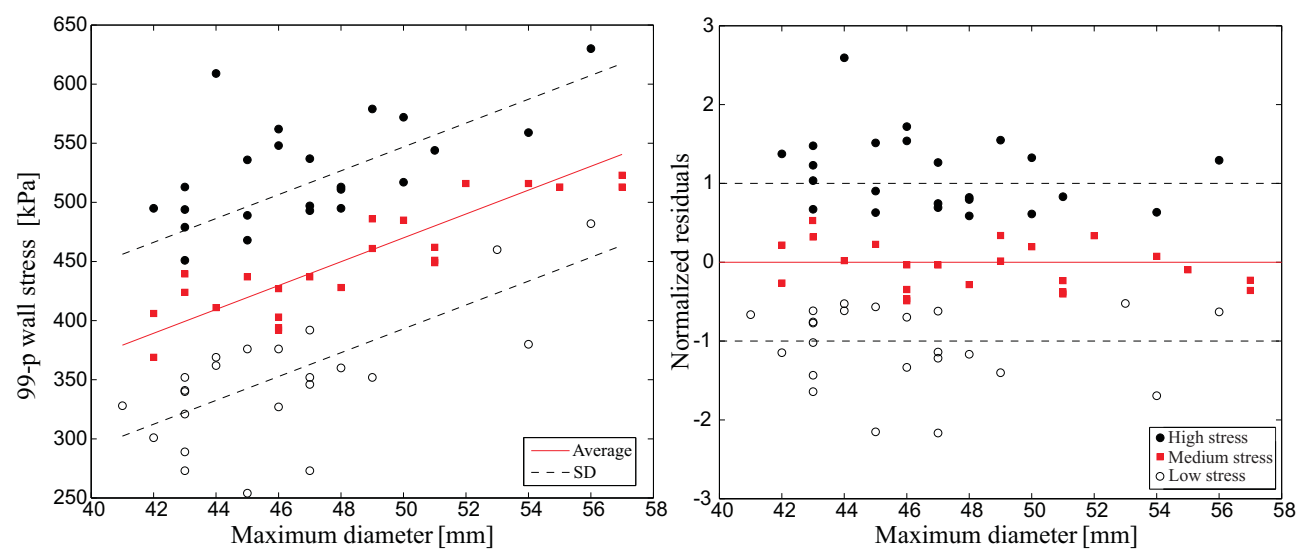

Figure 6.2: Regression model between maximum aortic diameter and 99-percentile wall stress $(r=0.47, p$-value $<0.01$, left figure). The residuals of the regression model, divided by the standard deviation (SD, right figure).

Patient demographics of the three stress groups are summarized in table 6.1. Gender was significantly different between the groups, as all female patients had a relative high AAA wall stress. The relative thrombus volume and calcification index was not significantly different between the groups (p-values 0.16 and 0.24 , respectively). The AAA growth rate per group is displayed in the box-and-whisker plot in figure 6.3. The growth rate of the low stress group was significantly lower than of the medium stress group (Kruskal-Wallis test, p-value 0.02). The difference in growth rate between the low and high wall stress groups was close to significant (Kruskal-Wall test, p-value $0.08)$.

Biomarker concentrations were determined in 18 patients, with in total 46 measurements (see figure 6.1). Determined biomarker levels were inaccurate and discarded for three TIMP- 1 measurements and the data of six hs-CRP and $\alpha 1$-AT analyses were missing. The biomarker concentrations for relative low, medium and high stress groups are given in table 6.2. P-values were computed with ANOVA, using the log transformed concentrations. The mean plots of the MMP-9 and hs-CRP levels are displayed in figure 6.4. Although the average levels of MMP-9 and hs-CRP show an increasing trend with the relative wall stress, no significant differences were found in the log levels of any of the biomarkers between the three stress groups.

Partial correlations between absolute 99-percentile wall stress and the log levels of the biomarkers are shown in table 6.3. Significant correlations between MMP-9 and TIMP-1 (r -0.38, p-value 0.03), MMP-9 and hs-CRP (r 0.32, p-value 0.01), and TIMP1 and $\alpha 1$-AT $(\mathrm{r}-0.55$, p-value $<0.01)$ were found. AAA wall stress did not correlation with any of the biomarker. 
Table 6.1: Demographics for relative low $(n=23)$, medium $(n=23)$ and high $(n=23)$ stress AAA's.

\begin{tabular}{lcccc}
\hline \hline Relative wall stress & Low & Medium & High & p-value \\
\hline Gender (M:F) & $23: 0$ & $23: 0$ & $18: 5$ & $<0.01$ \\
Age (mean(SD)) years & $73(6)$ & $72(6)$ & $70(6)$ & $0.31^{*}$ \\
Smoking (never:ex:current) & $1: 20: 2$ & $3: 14: 6$ & $4: 13: 6$ & 0.17 \\
Hypertension ${ }^{1}$ & 11 & 13 & 16 & 0.50 \\
Statin use & 13 & 7 & 13 & 0.20 \\
Diabetes Mellitus Type I & 1 & 2 & 0 & 0.32 \\
Max diameter (mean(SD)) mm & $46(4)$ & $49(4)$ & $47(4)$ & $0.14^{*}$ \\
Relative thrombus volume (mean(SD)) $\%$ & $23(29)$ & $39(29)$ & $34(30)$ & $0.16^{*}$ \\
Calcification index (mean(SD)) \% & $20(16)$ & $19(15)$ & $13(9)$ & $0.24^{*}$ \\
AAA growth rate (median(IQR)) mm/y & $0(3)$ & $3(7.5)$ & $3(8.3)$ & $0.06+$ \\
\hline Pearson chi-square test unless stated otherwise & & & \\
${ }^{*}$ Independent t-test, +Kruskal-Wallis test & & & \\
${ }^{1}$ A consistent pressure more than 140 mmHg systolic or 90 mmHg diastolic & \\
\hline
\end{tabular}

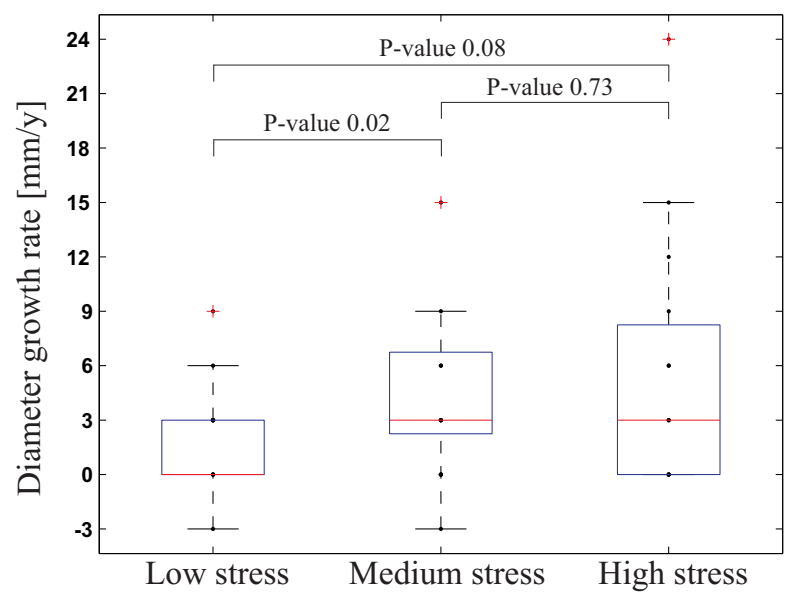

Figure 6.3: Box-and-Whisker plot of the AAA diameter growth rate for low, medium and high stress groups with the Kruskal-Wallis test $p$-values. The overall $p$-value was 0.06 for growth rate between all groups (Kruskal-Wallis test). 
Table 6.2: Biomarker concentrations (median (IQR)) for low, medium and high stress groups and for all AAA's. The number of measurements is indicated per biomarker and stress group. ANOVA p-values for the log levels of the biomarkers between the stress groups are given.

\begin{tabular}{lccccc}
\hline \hline Wall stress & Low & Normal & High & All & p-value \\
\hline MMP-9 & $34(22-38)$ & $43(22-64)$ & $49(24-53)$ & $41(22-50)$ & 0.52 \\
$(\mathrm{ng} / \mathrm{mL})$ & $(\mathrm{n}=18)$ & $(\mathrm{n}=16)$ & $(\mathrm{n}=12)$ & $(\mathrm{n}=46)$ & \\
TIMP-1 & $129(108-159)$ & $134(98-163)$ & $130(91-151)$ & $131(98-163)$ & 0.54 \\
$(\mathrm{ng} / \mathrm{mL})$ & $(\mathrm{n}=17)$ & $(\mathrm{n}=15)$ & $(\mathrm{n}=11)$ & $(\mathrm{n}=43)$ & \\
$\mathrm{hsCRP}$ & $2.3(1.4-3.1)$ & $\begin{array}{c}3.3(1.4-5.0) \\
(\mathrm{m}=17)\end{array}$ & $\begin{array}{c}3.7(1.2-6.1) \\
(\mathrm{n}=9)\end{array}$ & $\begin{array}{c}3.0(1.3-4.2) \\
(\mathrm{n}=40)\end{array}$ & 0.67 \\
$\begin{array}{c}(\mathrm{n}=14) \\
(\mathrm{g} / \mathrm{AT}\end{array}$ & $\begin{array}{c}1.6(1.2-1.8) \\
(\mathrm{n}=14)\end{array}$ & $\begin{array}{c}1.6(1.5-1.8) \\
(\mathrm{n}=17)\end{array}$ & $\begin{array}{c}1.6(1.5-1.8) \\
(\mathrm{n}=9)\end{array}$ & $\begin{array}{c}1.6(1.5-1.8) \\
(\mathrm{n}=40)\end{array}$ & 0.88 \\
\hline
\end{tabular}
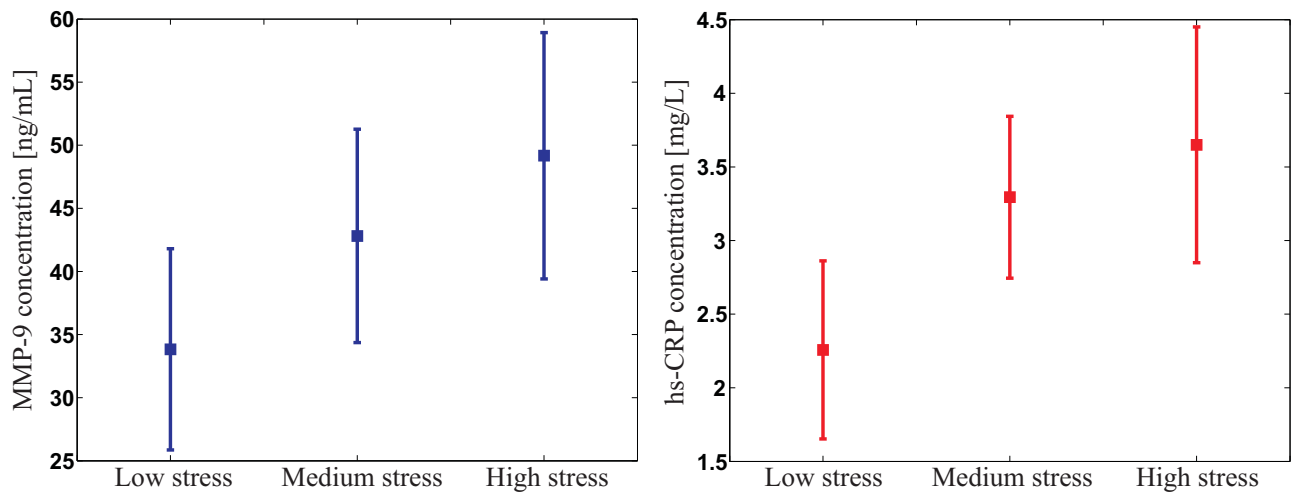

Figure 6.4: Means and Tukey HSD confidence intervals of the MMP-9 (left) and hsCRP (right) concentrations for low, medium and high stress groups. ANOVA p-values for the log transformed concentrations were 0.52 and 0.67 , respectively.

Table 6.4 shows the partial correlations between AAA growth rate and log levels of the biomarkers. It must be noted that the correlation coefficients between the biomarkers mutually in tables 6.3 and 6.4 do not exactly coincide due to the fact that partial correlations were computed. By doing so, the correlation between two variables is computed after adjusting for the relations with other variables included in the evaluation. Besides the previously found significant correlations between the biomarkers mutually, AAA growth rate showed to be significantly related to MMP-9 plasma concentration ( $\mathrm{r} 0.32$, p-value 0.05 ). This was also significant for the univariate correlation between AAA growth rate and MMP-9 concentration (r 0.34, p-value 0.02). 
Table 6.3: Partial correlation coefficient matrix between the wall stress and log levels of MMP-9, TIMP-1, hs-CRP and $\alpha 1-A T$. The upper right part shows the correlation coefficients and the number of measurements, the lower left part shows the corresponding $p$-values. The * indicates a significant correlation.

\begin{tabular}{|c|c|c|c|c|c|}
\hline & Stress & MMP-9 & TIMP-1 & hs-CRP & $\alpha+\alpha 1-\mathrm{AT}$ \\
\hline Stress & - & $\begin{array}{c}0.20 \\
(\mathrm{n}=46)\end{array}$ & $\begin{array}{c}0.06 \\
(n=41)\end{array}$ & $\begin{array}{c}0.02 \\
(\mathrm{n}=39)\end{array}$ & $\begin{array}{c}0.05 \\
(\mathrm{n}=40)\end{array}$ \\
\hline MMP-9 & 0.19 & - & $\begin{array}{l}-0.38^{*} \\
(\mathrm{n}=38)\end{array}$ & $\begin{array}{c}0.32^{*} \\
(\mathrm{n}=36)\end{array}$ & $\begin{array}{c}-0.16 \\
(n=37)\end{array}$ \\
\hline TIMP-1 & 0.73 & 0.03 & - & $\begin{array}{c}0.39 \\
(\mathrm{n}=32)\end{array}$ & $\begin{array}{l}-0.55^{*} \\
(\mathrm{n}=33)\end{array}$ \\
\hline hs-CRP & 0.92 & 0.01 & 0.09 & - & $\begin{array}{c}0.17 \\
(\mathrm{n}=39)\end{array}$ \\
\hline$\alpha 1-\mathrm{AT}$ & 0.77 & 0.37 & $<0.01$ & 0.32 & - \\
\hline
\end{tabular}

Table 6.4: Partial correlation coefficient matrix between AAA growth and $\log$ levels of MMP-9, TIMP-1, hs-CRP and $\alpha 1-A T$. The upper right part shows the correlation coefficients and the number of measurements, the lower left part shows the corresponding $p$-values. The * indicates a significant correlation.

\begin{tabular}{|c|c|c|c|c|c|}
\hline & Growth & MMP-9 & TIMP-1 & hsCRP & $\alpha 1-\mathrm{AT}$ \\
\hline Growth & - & $\begin{array}{c}0.32^{*} \\
(\mathrm{n}=46)\end{array}$ & $\begin{array}{c}0.12 \\
(\mathrm{n}=41)\end{array}$ & $\begin{array}{c}0.06 \\
(n=39)\end{array}$ & $\begin{array}{c}0.00 \\
(n=40)\end{array}$ \\
\hline MMP-9 & 0.05 & - & $\begin{array}{l}-0.39^{*} \\
(\mathrm{n}=38)\end{array}$ & $\begin{array}{c}0.40^{*} \\
(\mathrm{n}=36)\end{array}$ & $\begin{array}{c}-0.14 \\
(n=37)\end{array}$ \\
\hline TIMP-1 & 0.51 & 0.02 & - & $\begin{array}{c}0.38 \\
(\mathrm{n}=32)\end{array}$ & $\begin{array}{l}-0.55^{*} \\
(\mathrm{n}=33)\end{array}$ \\
\hline hs-CRP & 0.72 & 0.02 & 0.08 & - & $\begin{array}{c}0.17 \\
(\mathrm{n}=39)\end{array}$ \\
\hline$\alpha 1-\mathrm{AT}$ & 0.99 & 0.42 & $<0.01$ & 0.31 & - \\
\hline
\end{tabular}




\subsection{Discussion}

In this study we hypothesized that in AAA's with high wall stress, relative to the diameter, the wall is more extensively damaged and degenerated than average. This might be reflected by an increased AAA growth rate and up- or downregulation of specific biomarkers. The results showed that a relative medium or high wall stress could be associated with a higher growth rate, which was significant between the medium and low wall stress groups (p-value 0.02), and close to significant between the high and low wall stress groups (p-value 0.08). Although the average levels of MMP-9 and hs-CRP showed an increasing trend for the increasing relative wall stress groups, none of the MMP-9, TIMP-1, $\alpha 1$-AT and hs-CRP concentrations were significantly different between the stress groups (table 6.2). The average concentration of hs-CRP in the medium and high stress group exceeded $3 \mathrm{mg} / \mathrm{L}$, which was previously identified as threshold level between average and higher relative risk for future vascular events (Ridker and Silvertown, 2008). The growth rate in these groups was also significantly higher than in the low stress group. The MMP-9 concentration positively correlated with AAA growth (r 0.32, p-value 0.05), but no relation could be identified between absolute AAA wall stress and any of the biomarker concentrations.

The maximum aneurysm diameter and the corresponding 99-percentile wall stress showed a significant positive relation ( $\mathrm{r} 0.47$, p-value $<0.01$ ). The relative low, medium and high wall stress groups, as determined from this stress-diameter relation, matched for age, smoking, hypertension, use of statins and diabetes mellitus. The maximum AAA diameter, relative thrombus volume and calcification index were also not different between the wall stress groups. The fact that all female patients resided in the high stress group may be explained by geometrical differences in AAA's between men and women. Due to a smaller initial aortic diameter, AAA's of equal size have a greater proportional dilation in females than in males (Fillinger et al., 2004), which generally results in a stronger curvature at inflection points on the wall surface. As in most cases high stress areas are situated at inflection points (Speelman et al., 2008), this can result in a higher 99-percentile wall stress. A larger patient population should be evaluated to elaborate on the differences in wall stress between male and female patients.

Between the biomarkers mutually, a positive correlation was found between MMP9 and hs-CRP concentrations (tables 6.3 and 6.4), possibly reflecting the fact that both biomarkers respond to AAA related events; namely inflammation and matrix degradation. Furthermore, TIMP-1 was negatively correlated with MMP-9. Normally, TIMP-1 regulates the activity of MMP-9. This regulation may be disturbed in patients with AAA, resulting in a lower TIMP-1 concentration for higher MMP-9 levels. TIMP-1 and $\alpha 1-\mathrm{AT}$ were also negatively correlated. The pathophysiological meaning is not clear, but it was previously postulated that both MMP-9 and $\alpha 1$-AT positively correlated with AAA growth (Lindholt et al., 2000; Vega de Céniga et al., 2009). However, in the current study, only MMP-9 showed a positive correlation with AAA growth. Future research is required to establish the underlying relations between these biomarkers and their role in AAA pathophysiology. 
This study is the first to couple AAA biomechanics and biomarker information. Nevertheless, some limitations and future research suggestions need to be mentioned. First, all measurements in this study are treated as individual and independent, although repeated measurements were performed in the patient group. It may well be that the stress and biomarker information is not independent per patient. The analyses were repeated with only one, randomly selected measurement per patient and the same trends were identified. However, no significant correlations could be found due to the small sample size. Also, ANOVA repeated measurement analyses were performed with all patients with four consecutive CTA measurements. Again, the same trends were found, but due to the small sample size, no significance was reached in most cases. As the same trends were found in the single and repeated measurement analyses, we are confident that the stress and biomarker measurements can be treated as independent.

The AAA growth rate in this research was determined based on the maximum anterior-posterior diameter, which is currently the gold standard to determine the size of an AAA. The 3D models could be used to determine more sophisticated growth measures, based on the largest diameter perpendicular to the central axis or the AAA volume. However, these measures showed a considerable variation, in the same order as the growth rate itself. The determination of the maximum diameter is also subject to measuring errors and user variations. Future developments in 3D analysis tools may lead to more accurate and reproducible AAA growth rates.

Although it was previously shown that wall stress is a potentially better AAA rupture risk criterion than the maximum diameter (Fillinger et al., 2002; Truijers et al., 2007), AAA wall stress, as a stand-alone marker, may not be specific enough. Wall thickness variation, material heterogeneity, intraluminal thrombus and aortic calcifications were not included in the present simulations. Currently, no noninvasive techniques are available that can give information on the local wall thickness and material behavior. Implementation of thrombus and calcifications is debatable as the material properties, material model and interactions between thrombus, calcifications and AAA wall remain unknown (Speelman et al., 2007, 2009b). Including these patient-specific factors may increase the specificity of wall stress simulations in the future.

Most biomechanical considerations of AAA's have focused on computing stresses acting on the aneurysm wall and not on the wall strength. This is, however, an equally important part in rupture risk prediction, since rupture occurs when the stresses exceed the strength of the wall. Information on the local wall constituents, for instance by means of MRI or advanced molecular imaging may substantially contribute to wall strength estimations and therefore to future AAA rupture risk analysis.

It must be noted that the relations found in this study only apply for AAA's with diameters between 40 and $55 \mathrm{~mm}$, and cannot be extrapolated to smaller or larger AAA's. The current analysis was limited by a relative small patient group, representing AAAs with the most clinically relevant diameter. Extending the study with AAA's with a wider range in diameter may give more insight in the general relations between wall stress, AAA growth rate and biomarkers. 


\subsection{Conclusions}

To our knowledge, this is the first study that combines both circulating biomarker information and wall stress information with the prospective AAA growth rate. The MMP-9 plasma concentration positively correlated to the growth rate of the AAA's. A relative medium or high wall stress was associated with a higher growth rate. Although the average concentrations of MMP-9 and hs-CRP showed an increasing trend for higher relative wall stress, no significant correlation could be identified between absolute or relative wall stress and biomarker concentrations. Further analysis is warranted to verify the relation between AAA wall stress, growth rate and biomarker concentrations. 


\section{Chapter 7}

\section{General Discussion}

Computing wall stress on abdominal aortic aneurysms (AAA) is more and more performed to estimate AAA rupture risk. It has previously been shown that AAA wall stress has a higher sensitivity and specificity than maximum AAA diameter when it comes to rupture risk estimation (Fillinger et al., 2003). Automated AAA segmentation and meshing software has been developed to facilitate introduction of AAA wall stress analysis as a clinical diagnostic tool. However, reproducibility and robustness of wall stress analyses needs to be evaluated first. Additionally, the effect of simplifications like initial stress, thrombus and calcifications on the patient-specific wall stress has not been established.

The objective of this thesis was to optimize and standardize AAA wall stress analyses for future diagnostic purposes by performing patient-specific wall stress analyses at different levels of complexity and to evaluate the relation between AAA wall stress, AAA growth rate, and biomarker concentrations. In this chapter, an overview will be given of the studies presented in this thesis. Subsequently, the results will be viewed in a broader perspective and future recommendations will be given. 


\subsection{Introduction}

The risk of abdominal aortic aneurysm (AAA) rupture is nowadays mainly estimated based on the maximum diameter of the dilated aorta and, to a lesser extent, the rate with which the maximum diameter increases over time (Greenhalgh et al., 1998). However, from previous research it becomes clear that the maximum AAA diameter in some cases underestimates the risk of rupture, leading to unexpected ruptures, while in other cases the risk is overestimated (Darling et al., 1977; Lederle et al., 2002). The AAA may in that case be unnecessarily repaired, with all accompanying surgical risks.

From an engineering perspective, rupture of an AAA wall occurs when the wall stress surpasses the strength of the wall locally. Wall stress may also have an effect on the growth rate of an AAA, as an increase in wall stress may lead to an increase in the amount of damage to the AAA wall, which may lead to remodeling and growth of the vessel. The stress in the AAA wall as a result of the blood pressure is partly determined by the maximum diameter, but the complete geometry of the AAA also plays an important role (Hua and Mower, 2001; Vorp and vande Geest, 2005). AAA wall stress analyses, based on 3D imaging of AAA's, is therefore focus of a growing research area (de Putter et al., 2007; Fillinger et al., 2002; Papaharilaou et al., 2007; Truijers et al., 2007; Venkatasubramaniam et al., 2004; Wolters et al., 2005). Early patient-specific wall stress computations have shown that peak wall stress for ruptured and symptomatic AAA's was significantly higher than for electively repaired or asymptomatic AAA's (Fillinger et al., 2002; Truijers et al., 2007). In most cases, manual segmentation of the AAA is performed and labor-intensive procedures are followed to translate the image data to a finite element model that can be used to compute wall stresses on. Also, as in every model, simplifications and assumptions are made concerning factors that are difficult or impossible to determine, like local wall thickness and initial wall stress. The incorporation of thrombus and calcifications is also ignored in most cases as the correct implementation in the analysis is not known. It is unclear to what extent these simplifications or assumptions influence the resulting wall stress on a patient-specific basis. This, and the fact that the derivation of the $3 \mathrm{D}$ model is complex and labor-intensive, dissuades an easy translation to a clinical environment. The objective of this thesis was to optimize and standardize AAA wall stress analyses for future diagnostic purposes by performing patient-specific wall stress analyses at different levels of complexity and to evaluate the relation between AAA wall stress, AAA growth rate, and biomarker concentrations.

For the purpose of a future introduction of wall stress analyses as a clinical diagnostic tool, Philips Healthcare (Best, the Netherlands) developed a software package HemoDyn, in collaboration with the University Medical Center Utrecht (the Netherlands), Philips Research (Paris, France), Eindhoven University of Technology, and our group (Breeuwer et al., 2008; de Putter et al., 2007; Delingette, 1994; Gérard et al., 2002; Ollabarriaga et al., 2005). This package is developed within the Philips ViewForum environment, which is widely used for visualization and analysis of medical images within clinical centers. HemoDyn is used for automatic segmentation and meshing of AAA's in the major part of the research in this thesis. 


\subsection{Summary and Conclusions}

HemoDyn was created to decrease the user input in the segmentation procedure, and can be used to create a finite element mesh and to compute AAA wall stress (Breeuwer et al., 2008). Based on the selection of only three points in the lumen of the AAA in the CTA data of the patient (one starting point at the level of the lowest renal branch and two end points in the iliac arteries, proximal to the aortic bifurcation), automatic centerline tracking and subsequent 3D segmentation of the lumen and the thrombus is performed. This highly automated process should increase the stability and reproducibility of the wall stress analyses. Heng et al. (2008) studied inter- and intra-operator reliability of peak wall stress, based on manual contour segmentation of 10 AAA's. Inter-operator variation was found over $100 \%$ between the lowest and highest stress value for the same patient. A double analysis on 10 patients by one operator resulted in differences up to $40 \%$. In chapter 2 , reproducibility of the AAA wall stress was determined using HemoDyn, by evaluating CTA data of 20 patients by three different operators. Two operators performed the analyses 5 times. The intra-operator intraclass correlation coefficient (ICC) of the peak wall stress was 0.73 and 0.79 , while the inter-operator ICC was 0.71 . This approached the findings by Heng et al. (2008). The 99-percentile wall stress proved to be a more reproducible wall stress parameter than peak wall stress (intra-operator ICC 0.94, 0.94 and interoperator ICC 0.95). The 99-percentile wall stress is computed as the highest wall stress in the AAA wall after exclusion of $1 \%$ of the wall, containing the highest stresses. Clearly, small subtle shape changes induced by the segmentation of different operators, strongly influenced the peak wall stress, while this effect was significantly lower for the 99-percentile wall stress. A strong linear relation between the 99-percentile wall stress and the maximum AAA diameter was identified, indicating that 99-percentile wall stress can still discriminate between AAA's of different sizes. However, a future patient study, comparing the 99-percentile wall stress for ruptured and non-ruptured AAA's is required to establish the clinical relevance of the 99-percentile wall stress in the rupture risk stratification.

During CTA imaging, the AAA is subjected to a time-averaged blood pressure and is therefore not stress free. However, until now, all patient-specific wall stress studies ignored this fact and applied a systolic blood pressure directly to the geometry derived from the CTA data. The Backward Incremental (BI) method was introduced by de Putter et al. (2007) to account for the initial stress in AAA's. De Putter et al. (2007) validated the wall stress as computed by finite element analyses and Merkx et al. (2009) showed that when using the BI method, the wall motion from the wall stress analysis significantly better corresponded to the gated MRI wall motion for ten patients. In chapter $\mathbf{3}$ of this thesis, the effect of neglecting the initial stress on the AAA wall stress of patient-specific AAA models was studied using the BI method. Wall stress was computed with and without initial stress using both a linear and nonlinear material model for the AAA wall and the results clearly showed 
that ignoring initial stress and material nonlinearity not only leads to a changed wall stress, but also that this change was different for each patient. Therefore, no general correction factor could be used to estimate the effect on the wall stress and in future wall stress studies initial wall stress and material nonlinearity cannot be omitted.

Two factors that may influence AAA wall stress can distinctly be identified from the CTA data, which are intraluminal thrombus and calcifications. In chapter 4, the mechanical role of thrombus was evaluated by computing wall stress with and without thrombus in idealized axisymmetric AAA models with various thrombus stiffnesses and volumes, and in 30 patient-specific AAA models. Additionally, the growth rate of these AAA's was prospectively monitored and a comparison was made between the growth rate of AAA's with relative small and large thrombi. The results showed that a large thrombus was associated with lower wall stress, but also with a higher growth rate. This indicates that weakening of the AAA wall, under the influence of thrombus, may play a more imminent role in the process of AAA growth, than the AAA wall stress (vande Geest et al., 2006c; Vorp et al., 2001).

Chapter $\mathbf{5}$ discusses the effect of calcifications in the AAA wall on patient-specific wall stress. The location of calcifications was determined from CTA data and projected on the finite element models by changing the material properties locally at areas of calcifications. Wall stress was computed with and without calcifications and stresses were found to be increased locally at calcification sites. However, the effect of calcifications on the peak wall stress was also determined by the location, shape, and elastic modulus of the calcifications and cannot be determined solely on the amount of calcification present in the wall.

In chapter 6, a first step to clinical applicability of AAA wall stress analysis was made. AAA growth rate and biomarker concentrations in the blood of the patients are compared with the wall stress. It was hypothesized that AAA's with a high wall stress, relative to the average wall stress at the corresponding diameter, have increased damage and degeneration of the AAA wall. This could reflect on AAA growth and the up- or downregulation of specific circulating biomarkers. The 99percentile wall stress was determined with incorporation of the initial stress and a nonlinear material model for the AAA wall, but without thrombus and calcifications. A relative medium or high wall stress was indeed found to be associated with a higher AAA growth rate compared to AAA's with a relative low wall stress. Also, the matrix metalloproteinase-9 (MMP-9) concentration, which is involved in the breakdown of the extra cellular matrix, was positively related to AAA growth rate. Although the average concentrations of MMP-9 and C-reactive protein (a marker for inflammation) showed an increase for higher relative wall stress, no significant correlation was found between wall stress and biomarkers levels analyzed in this study. Further analysis is warranted to verify the relation between AAA wall stress, diameter expansion and biomarker concentrations.

Returning to the objective of this thesis, it can be concluded that wall stress analyses are partly optimized and standardized by proposing a robust and reproducible stress measure in the form of the 99-percentile wall stress. The effect of initial stress and material nonlinearity was determined and it was concluded that they are indispensable in future wall stress analyses. Despite the fact that thrombus and calcifications have 
their specific influence on the resulting wall stress per AAA, additional research is required to fully understand the mechanical effects of both factors. Future research should elaborate on the correct mechanical models of thrombus and calcifications and on the implementation in the finite element models, preferably using non-invasive imaging modalities like ultrasound or dynamic MRI or CTA.

\subsection{Scope and Limitations}

The objective of this thesis was to optimize and standardize AAA wall stress analyses for future diagnostic purposes. These purposes may include an estimation parameter for AAA rupture risk, in addition to the currently used decision parameters, but also the prediction of the AAA growth rate. A dynamic follow up plan can then be developed based on the computed wall stress. Some patients may require more frequent hospital visits, while others, as their AAA remains stable over a longer period, can suffice with long interval follow up.

The first step to clinical applicability of wall stress is set in this thesis. A robust and reproducible stress measure is proposed and important factors influencing the wall stress have been identified. Several limitations and assumptions remain that may improve the stress analysis. One of the major assumptions that is inevitable is that of a constant wall thickness over the whole aneurysm model. It is known that a strong variation in wall thickness exists between, but also within patients (Hellenthal et al., 2008; Raghavan et al., 2006a). Including the local variation in wall thickness can have a strong effect on the resulting wall stress. Currently, no non-invasive methods are available that can reliably determine the local wall thickness. CTA cannot provide information on the location of the thrombus-wall transition and therefore no wall thickness can be determined. Most MRI protocols can also not be used due to chemical shift artifacts, caused by the transition between water-rich and fat-rich tissues, and due to the large voxel size, relative to the expected wall thickness. Black-blood MRI sequences may reach sufficient resolution, but no discrimination between wall and thrombus can yet be made with this protocol, and thus no wall thickness can be measured in the presence of thrombus. Although ultrasound evaluation can be used to determine the local AAA wall thickness, the wall thickness cannot be determined over the whole AAA. Using intravascular ultrasound (IVUS), a complete wall thickness map can be made, but this medical imaging technique is expensive, time-consuming and is considered as an intervention. This makes IVUS unsuitable for follow-up of AAA patients. Developments in (3D) ultrasound imaging and advanced MRI sequences may in the future facilitate non-invasive local wall thickness measurements of AAA's.

Another limitation is the mechanical material model that is used for the AAA wall, the thrombus and the calcifications. It is extremely difficult to evaluate the properties of the constituents separately, especially for thrombus and calcifications. Extensive mechanical testing has resulted in a commonly used hyper-elastic, nonlinear material model for the AAA wall in general (Raghavan and Vorp, 2000). By using dynamic MRI measurements to determine the distensibility of the AAA and simultaneous 
pressure recordings, van 't Veer et al. (2008) determined patient-specific Young's moduli non-invasively. Future developments of this method may result in local nonlinear and even non-isotropic material properties, by evaluating the local wall deformation patterns instead of the total AAA volume. However, higher resolution imaging may be an important requisite for this analysis.

The results that are presented for the mechanical behavior of thrombus vary strongly. Van Dam et al. (2008) found thrombus shear moduli around $2 \mathrm{kPa}$, while Wang et al. (2001) presented up to $180 \mathrm{kPa}$ for thrombus. Also within these studies a wide spread in mechanical behavior was found between and within the tested thrombi. This hampers the use of a single shear modulus for thrombus in all patient-specific wall stress analyses. Improvements in dynamic CTA or MRI measurements may in the future give more insight in the variation in mechanical behavior of thrombus within patients, by evaluating the thrombus deformations over the cardiac cycle (Truijers et al., 2009). Using finite element modeling, an estimate of the patientspecific mechanical properties may be derived. In this and previous AAA wall stress studies, thrombus has been modeled as a solid structure. Modeling thrombus as a porous material may be more appropriate, considering the poro-elastic character of thrombus (Adolph et al., 1997; Boschetti et al., 2007). In that case, the pressure can be transported through the pores of the material. The effect that a poroelastic material model for the thrombus has on the wall stress cannot be predicted on forehand. Parameters like porosity and viscous permeability first need to be determined by experimental testing, before wall stress computations can be extended with a porous material model for thrombus.

Previously, research on calcifications using high resolution $\mu \mathrm{CT}$ revealed that what appears as a solid, uniform calcification in normal resolution CTA images, may in fact be a complex arrangement of smaller calcified areas (de Putter et al., 2006b). This means that it is of little use to accurately model the shape and the material interface of the calcification, based on the normal CTA image data. The resolution is too low to capture the required details. Future research should therefore focus on accurately establishing the material properties of calcified regions and on experimentally assessing the rupture potential of the calcifications, the material interface and the surrounding non-calcified tissue.

Most biomechanical considerations of AAA's have focused on computing stresses acting on the aneurysm wall, and not on the wall strength. This is, however, an equally important part in rupture risk prediction, since rupture occurs when the stresses exceed the strength of the wall. If only the wall stress is considered and a certain stress threshold is determined that indicates a high risk of rupture, this corresponds with the choice of a uniform failure strength throughout the AAA. vande Geest et al. (2006c) proposed a Rupture Potential Index (RPI), which combines the wall stress computed with finite element analysis and the wall strength, estimated with a seven parameter statistical model (age, gender, family history of AAA, smoking status, AAA size, local diameter, and local thrombus thickness). The results suggested that the peak RPI may be better able to identify those AAA's at high risk of rupture than maximum diameter or peak wall stress alone. Future developments in medical imaging quality to obtain local wall thickness may also add to this strength estimation. The clinical 
relevance of this method for rupture assessment has yet to be validated, however, its success could aid clinicians in decision making and AAA patient management (vande Geest et al., 2006a).

\subsection{Clinical Perspectives}

With the studies in this thesis, important steps were made to standardize AAA wall stress analyses. Although future research is required to further increase the accuracy of the wall stress analyses, the currently proposed model is close to be clinically applicable. However, the clinical relevance, which is much more important, has only been touched swiftly in this thesis. A significant positive stress-diameter relation was identified and it was shown that a relative high wall stress was associated with a higher growth rate. The ultimate question whether AAA rupture risk can be predicted based on wall stress cannot (yet) be answered. However, tools have been created, and important factors have been identified in the process of wall stress computations. The road is now free to investigate the clinical relevance of wall stress with respect to AAA rupture risk and growth. As more and more AAA patients in follow-up are followed using CTA instead of ultrasound, the basis of this study is already present. Continuous follow-up with CTA of a large group of patients can give insight in the relation of AAA wall stress and growth rate. As AAA rupture is a relative rare phenomenon, especially in the combination with recent prerupture CTA imaging, a large prospective patient study is required. The @neurist program can therefore be taken as an example (see www.aneurist.org). This program integrates biomedical information for the management of cerebral aneurysms. A large collaboration between multiple health organizations in several countries created an infrastructure for combining data of cerebral aneurysms with the goal to provide individualized aneurysm rupture predictions. Such an infrastructure may also be of high value in the rupture risk estimation of AAA's.

\subsection{General Conclusion}

Although the research in this thesis lays the basis for AAA wall stress to be incorporated in clinical diagnostics, the clinical relevance of AAA wall stress is still unknown. Based on the results presented here, one can conclude that a standardized AAA wall stress analysis using automatic segmentation is required. Additionally, initial stress and material nonlinearity are undisputable elements in the finite element simulations. To be able to conclude on the role of thrombus and calcifications, future research on the material properties and interface with the surroundings is required. Also, developments in the wall strength estimation are essential for an accurate risk estimation. With the foundation that has been laid in this thesis, the road is open for profound patient studies to evaluate the clinical relevance of AAA wall stress in relation to the AAA rupture risk and growth rate. 
Chapter 7 


\section{Appendix A}

\section{Finite Element Formulation of Mass and Momentum Balance Laws}

In all chapters of this thesis, the finite element method is used to compute AAA wall stress. To do so, the balance equations of momentum and mass are solved under specific boundary conditions, namely an intra-aortic pressure and fixation in space of a part of the model (the upper and lower planes of the model to simulate fixation to the rest of the vascular system). In chapters 3,4 , and 6 , also initial stresses are accounted for using the backward incremental method, as proposed by de Putter et al. (2007). In this appendix, the finite element formulation of the momentum and mass balance equations is derived and the differences between the conventional and the backward incremental method is outlined. 


\section{Deformation}

The aortic wall is considered as an incompressible solid material. The deformation of an incompressible solid is described by the balance equations of momentum and mass:

$$
\begin{cases}\nabla \cdot \boldsymbol{\sigma}=\mathbf{0} & \text { in } \Omega(t) \\ \operatorname{det}(\boldsymbol{F})-1=0 & \text { in } \Omega(t)\end{cases}
$$

with $\nabla$ the gradient operator, $\boldsymbol{\sigma}$ the Cauchy stress and $\boldsymbol{F}=\left(\nabla_{0} \boldsymbol{x}\right)^{T}$ the deformation gradient tensor, describing the deformation between the reference configuration $\Omega_{0}$ with position vectors $\boldsymbol{x}_{0}$ and the current configuration $\Omega(t)$ with position vectors $\boldsymbol{x}$ (see figure A.1). The Cauchy stress for an incompressible material is written as:

$$
\boldsymbol{\sigma}=-p \boldsymbol{I}+\boldsymbol{\tau}
$$

with $p$ the hydrostatic pressure, $\boldsymbol{I}$ the identity matrix and $\boldsymbol{\tau}$ the extra stress tensor resulting from deformation. $\tau$ is defined by the material model and will be discussed later on in this appendix.

Boundary conditions need to be applied to the complete boundary $\Gamma(t)$ of the volume $\Omega(t)$. For each part of the complete boundary, the boundary conditions can be of the form:

$$
\begin{aligned}
& \boldsymbol{x} \cdot \boldsymbol{n}=x_{n} \vee(\boldsymbol{\sigma} \cdot \boldsymbol{n}) \cdot \boldsymbol{n}=s_{n} \quad \text { on } \Gamma \\
& \boldsymbol{x} \cdot \boldsymbol{t}_{\boldsymbol{i}}=x_{t_{i}} \vee(\boldsymbol{\sigma} \cdot \boldsymbol{n}) \cdot \boldsymbol{t}_{\boldsymbol{i}}=s_{t_{i}} \quad(i=1,2) \quad \text { on } \Gamma
\end{aligned}
$$

where, in case of Dirichlet boundary conditions, $x_{n}$ and $x_{t_{i}}$ denote the prescribed displacement in the normal and two tangential directions and, in case of Neumann boundary conditions, $s_{n}$ and $s_{t_{i}}$ denote the normal and tangential components of the surface traction vector $\boldsymbol{s}=\boldsymbol{\sigma} \cdot \boldsymbol{n}$.

\section{Weak Form}

The first step towards the finite element formulation is to rewrite the conservation laws into integral equations. Therefore, the set of equations A.1 is transformed by means of the weighted residuals method (which states that for a function $g(x)=0$ on $\Omega$ the equivalent $\int_{\Omega} v(x) g(x) d x=0$ must hold for all weighting functions $v(x)$ ). Then, after integrating by parts and applying the divergence theorem, the following weak formulations are obtained (de Putter et al., 2007; van de Vosse et al., 2003):

$$
\left\{\begin{array}{l}
\int_{\Omega(t)}(\nabla \boldsymbol{w})^{T}: \boldsymbol{\sigma} \mathrm{d} \Omega=\int_{\Gamma(t)} \boldsymbol{w} \cdot(\boldsymbol{\sigma} \cdot \boldsymbol{n}) \mathrm{d} \Gamma \\
\int_{\Omega(t)} q(J-1) \mathrm{d} \Omega=0
\end{array}\right.
$$


In this set of equations, $\boldsymbol{w}$ and $q$ are weighting functions for the momentum and mass equations, which are continuous functions on the integral domain $\Omega$. The left hand side of the momentum equation represents the internal forces and the right hand side the external forces on the body surface. In the mass equation, $\operatorname{det}(\boldsymbol{F})$ is replaced by $J$.

The integrals are defined with respect to the current (unknown) configuration $\Omega(t)$ with boundary $\Gamma(t)$. A transformation to a known configuration is required to solve the system. Following the Updated Lagrange approach, the integrals are transformed to the last known configuration. As the pressure will be increased stepwise, this is generally the last converged configuration $\Omega_{n}$ (figure A.1).

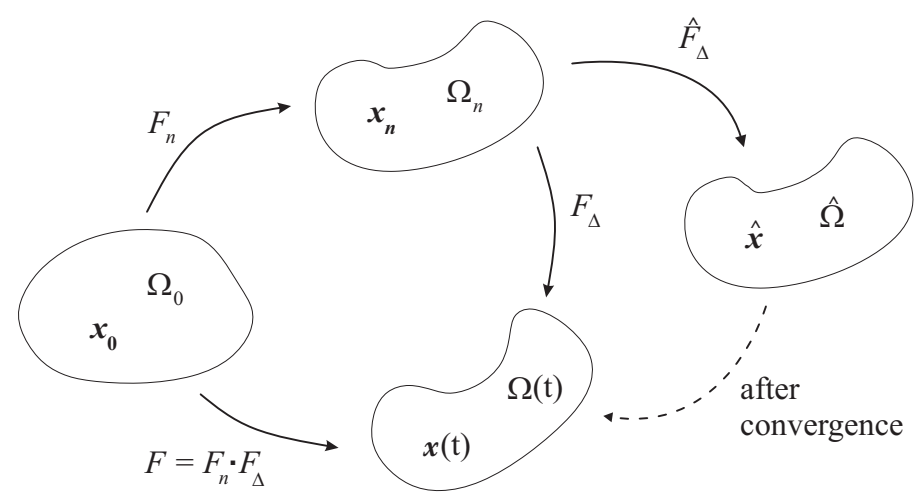

Figure A.1: The Updated Lagrange scheme. $\Omega_{0}$ is the reference configuration with position vector $\boldsymbol{x}_{\mathbf{0}}$. The boundary conditions are applied stepwise until the final solution $\Omega(t)$ has been reached. $\Omega_{n}$ is an intermediate, converged solution and $\hat{\Omega}$ is a new, unconverged solution in the iteration process of the present step. This configuration forms $\Omega(t)$ when convergence in the iteration process has been reached.

\section{Updated Lagrange Method}

Following figure A.1 we define:

$$
\begin{aligned}
& \boldsymbol{F}_{n}=\left(\nabla_{0} \boldsymbol{x}_{n}\right)^{T} \\
& \boldsymbol{F}_{\Delta}=\left(\nabla_{n} \boldsymbol{x}\right)^{T} \\
& \boldsymbol{F}=\boldsymbol{F}_{\Delta} \cdot \boldsymbol{F}_{n}
\end{aligned}
$$


Here, $\nabla_{n}$ denotes the gradient operator with respect to $\Omega_{n}$. The gradient operator with respect to $\Omega(t)$ can be written as $\nabla=\boldsymbol{F}_{\Delta}^{-T} \cdot \nabla_{n}{ }^{1}$. Transformation of the weak form A.4 from $\Omega(t)$ to $\Omega_{n}$ then yields:

$$
\left\{\begin{array}{l}
\int_{\Omega_{n}}\left(\nabla_{n} \boldsymbol{w}\right)^{T}: \boldsymbol{F}_{\Delta}^{-1} \cdot \boldsymbol{\sigma} J_{\Delta} \mathrm{d} \Omega_{n}=\int_{\Gamma_{n}} \boldsymbol{w} \cdot\left(\boldsymbol{\sigma} \cdot\left(\boldsymbol{F}_{\Delta}^{-T} \cdot \boldsymbol{n}_{n}\right)\right) J_{\Delta} \mathrm{d} \Gamma_{n} \\
\int_{\Omega_{n}} q(J-1) J_{\Delta} \mathrm{d} \Omega_{n}=0
\end{array}\right.
$$

with $\mathrm{d} \Omega=J_{\Delta} \mathrm{d} \Omega_{n} . J_{\Delta}=\operatorname{det}\left(\boldsymbol{F}_{\Delta}\right)$ is the relative volume change between $\Omega(t)$ and $\Omega_{n}$.

\section{Linearization}

The resulting set of equations in A.6 is nonlinear due to the multiplications of multiple displacement-dependent factors. Newton linearization is therefore applied to the equations. Let $(\hat{\boldsymbol{x}}, \hat{p})$ be the estimates of the last unconverged solution, for the displacement and pressure $(\boldsymbol{x}, p)$. The errors $(\delta \boldsymbol{x}, \delta p)$ are then given by:

$$
\begin{aligned}
& \delta \boldsymbol{x}=\boldsymbol{x}-\hat{\boldsymbol{x}} \\
& \delta p=p-\hat{p}
\end{aligned}
$$

All quantities of the integrands in equations A.6 can be expanded with respect to $(\delta \boldsymbol{x}, \delta p)$. The following expansions were used:

$$
\boldsymbol{F}_{\Delta}^{-1}=\hat{\boldsymbol{F}}_{\Delta}^{-1}+\delta\left(\boldsymbol{F}_{\Delta}^{-1}\right) ; \quad \boldsymbol{\sigma}=\hat{\boldsymbol{\sigma}}+\delta \boldsymbol{\sigma} ; \quad J_{\Delta}=\hat{J}_{\Delta}+\delta J_{\Delta} ; \quad J=\hat{J}+\delta J
$$

Substitution of these expressions and neglecting double error products leads to:

$$
\left\{\begin{array}{l}
\int_{\Omega_{n}}\left(\nabla_{n} \boldsymbol{w}\right)^{T}:\left[\delta\left(\boldsymbol{F}_{\Delta}^{-1}\right) \cdot \hat{\boldsymbol{\sigma}} \hat{J}_{\Delta}+\hat{\boldsymbol{F}}_{\Delta}^{-1} \cdot\left(\hat{\boldsymbol{\sigma}} \delta J_{\Delta}+\delta \boldsymbol{\sigma} \hat{J}_{\Delta}\right)\right] \mathrm{d} \Omega_{n}- \\
\int_{\Gamma_{n}} \boldsymbol{w} \cdot\left[\delta \boldsymbol{\sigma} \cdot \hat{\boldsymbol{F}}_{\Delta}^{-T} \cdot \boldsymbol{n}_{n} \hat{J}_{\Delta}+\hat{\boldsymbol{\sigma}} \cdot \delta\left(\boldsymbol{F}_{\Delta}^{-T}\right) \cdot \boldsymbol{n}_{n} \hat{J}_{\Delta}+\hat{\boldsymbol{\sigma}} \cdot \hat{\boldsymbol{F}}_{\Delta}^{-T} \cdot \boldsymbol{n}_{n} \delta J_{\Delta}\right] \mathrm{d} \Gamma_{n}= \\
-\int_{\Omega_{n}}\left(\nabla_{n} \boldsymbol{w}\right)^{T}: \hat{\boldsymbol{F}}_{\Delta}^{-1} \cdot \hat{\boldsymbol{\sigma}} \hat{J}_{\Delta} \mathrm{d} \Omega_{n}+\int_{\Gamma_{n}} \boldsymbol{w} \cdot\left(\hat{\boldsymbol{\sigma}} \cdot\left(\hat{\boldsymbol{F}}_{\Delta}^{-T} \cdot \boldsymbol{n}_{n}\right)\right) \hat{J}_{\Delta} \mathrm{d} \Gamma_{n} \\
\int_{\Omega_{n}} q\left((\hat{J}-1) \delta J_{\Delta}+\delta J \hat{J}_{\Delta}\right) \mathrm{d} \Omega_{n}=-\int_{\Omega_{n}} q(\hat{J}-1) \hat{J}_{\Delta} \mathrm{d} \Omega_{n}
\end{array}\right.
$$

\footnotetext{
${ }^{1}$ Use $\nabla \boldsymbol{x}=\boldsymbol{I}=\boldsymbol{F}_{\Delta}^{-T} \cdot \boldsymbol{F}_{\Delta}^{T}=\boldsymbol{F}_{\Delta}^{-T} \cdot \nabla_{n} \boldsymbol{x}$
} 
Note that the right hand side of the first equation expresses the imbalance in momentum in the estimated configuration, as a consequence of the error terms $\delta\left(\boldsymbol{F}_{\Delta}^{-1}\right), \delta J_{\Delta}$ and $\delta \boldsymbol{\sigma}$ in the left hand side. This imbalance is used to iteratively reduce the error terms. Commonly, in the iteration process, the left hand side is approximated by neglecting the surface integral. This may influence the convergence rate, but will not affect the final solution, since no approximation is performed in the right hand side.

The right hand side of the second equation expresses the imbalance in mass due to the errors in $\delta J$ and $\delta J_{\Delta}$. Here, the term with $\delta J_{\Delta}$ is commonly left out of the iteration process. To proceed, expressions for $\delta\left(\boldsymbol{F}_{\Delta}^{-1}\right), \delta J$ and $\delta \boldsymbol{\sigma}$ need to be formulated.

\section{Error $\delta\left(\boldsymbol{F}_{\Delta}^{-1}\right)$}

Using $\boldsymbol{F}_{\Delta} \cdot \boldsymbol{F}_{\Delta}^{-1}=\boldsymbol{I}$, it follows that:

$$
\begin{aligned}
\left(\hat{\boldsymbol{F}}_{\Delta}+\delta \boldsymbol{F}_{\Delta}\right) \cdot\left(\hat{\boldsymbol{F}}_{\Delta}^{-1}+\delta\left(\boldsymbol{F}_{\Delta}^{-1}\right)\right)= \\
\boldsymbol{I}+\hat{\boldsymbol{F}}_{\Delta} \cdot \delta\left(\boldsymbol{F}_{\Delta}^{-1}\right)+\delta \boldsymbol{F}_{\Delta} \cdot \hat{\boldsymbol{F}}_{\Delta}^{-1}+\delta \boldsymbol{F}_{\Delta} \cdot \delta\left(\boldsymbol{F}_{\Delta}^{-1}\right)=\boldsymbol{I}
\end{aligned}
$$

The double error product is neglected, which leads to the following expression:

$$
\begin{aligned}
\delta\left(\boldsymbol{F}_{\Delta}^{-1}\right) & =-\hat{\boldsymbol{F}}_{\Delta}^{-1} \cdot \delta \boldsymbol{F}_{\Delta} \cdot \hat{\boldsymbol{F}}_{\Delta}^{-1} \\
& =-\hat{\boldsymbol{F}}_{\Delta}^{-1} \cdot\left(\hat{\boldsymbol{F}}_{\Delta}^{-T} \cdot \delta \boldsymbol{F}_{\Delta}^{T}\right)^{T} \\
& =-\hat{\boldsymbol{F}}_{\Delta}^{-1} \cdot\left(\hat{\boldsymbol{F}}_{\Delta}^{-T} \cdot \nabla_{n} \delta \boldsymbol{x}\right)^{T} \\
& =-\hat{\boldsymbol{F}}_{\Delta}^{-1} \cdot(\hat{\nabla} \delta \boldsymbol{x})^{T} \\
& =-\hat{\boldsymbol{F}}_{\Delta}^{-1} \cdot \boldsymbol{H}_{\delta x}
\end{aligned}
$$

with $\boldsymbol{H}_{\delta x}=(\hat{\nabla} \delta \boldsymbol{x})^{T}$.

\section{Error $\delta J$}

The expression for $\delta J$ can be derived from the definition of $J^{2}$ :

$$
\begin{aligned}
\delta J & =\hat{J} \operatorname{tr}\left(\delta \boldsymbol{F} \cdot \hat{\boldsymbol{F}}^{-1}\right) \\
& =\hat{J} \operatorname{tr}\left(\left(\hat{\boldsymbol{F}}^{-T} \cdot \delta \boldsymbol{F}^{T}\right)^{T}\right) \\
& =\hat{J} \operatorname{tr}\left(\left(\hat{\boldsymbol{F}}^{-T} \cdot \nabla_{0} \delta \boldsymbol{x}\right)^{T}\right) \\
& =\hat{J} \operatorname{tr}\left((\hat{\nabla} \delta \boldsymbol{x})^{T}\right) \\
& =\hat{J} \operatorname{tr}\left(\boldsymbol{H}_{\delta x}\right)
\end{aligned}
$$

\footnotetext{
${ }^{2}$ use $J=\operatorname{det}(\boldsymbol{F})=\operatorname{det}(\hat{\boldsymbol{F}}+\delta \boldsymbol{F})=\operatorname{det}\left(\left(\boldsymbol{I}+\delta \boldsymbol{F} \cdot \hat{\boldsymbol{F}}^{-1}\right) \cdot \hat{\boldsymbol{F}}\right)=\operatorname{det}\left(\boldsymbol{I}+\delta \boldsymbol{F} \cdot \hat{\boldsymbol{F}}^{-1}\right) \operatorname{det}(\hat{\boldsymbol{F}}) \approx$ $\left(1+\operatorname{tr}\left(\delta \boldsymbol{F} \cdot \hat{\boldsymbol{F}}^{-1}\right)\right) \operatorname{det}(\hat{\boldsymbol{F}})=\hat{J}+\hat{J} \operatorname{tr}\left(\delta \boldsymbol{F} \cdot \hat{\boldsymbol{F}}^{-1}\right)$.
} 
Similarly:

$$
\delta J_{\Delta}=\hat{J}_{\Delta} \operatorname{tr}\left(\boldsymbol{H}_{\delta x}\right)
$$

After filling in the above expressions for $\delta\left(\boldsymbol{F}_{\Delta}^{-1}\right), \delta J$ and $\delta J_{\Delta}$ and rearranging the terms, the weak form of equations A.9 now reads:

$$
\left\{\begin{array}{l}
\int_{\Omega_{n}}\left(\nabla_{n} \boldsymbol{w}\right)^{T}: \hat{\boldsymbol{F}}_{\Delta}^{-1} \cdot\left[-\boldsymbol{H}_{\delta x} \cdot \hat{\boldsymbol{\sigma}}+\hat{\boldsymbol{\sigma}} \operatorname{tr}\left(\boldsymbol{H}_{\delta x}\right)+\delta \boldsymbol{\sigma}\right] \hat{J}_{\Delta} \mathrm{d} \Omega_{n}= \\
\quad-\int_{\Omega_{n}}\left(\nabla_{n} \boldsymbol{w}\right)^{T}: \hat{\boldsymbol{F}}_{\Delta}^{-1} \cdot \hat{\boldsymbol{\sigma}} \hat{J}_{\Delta} \mathrm{d} \Omega_{n}+\int_{\Gamma_{n}} \boldsymbol{w} \cdot\left(\hat{\boldsymbol{\sigma}} \cdot\left(\hat{\boldsymbol{F}}_{\Delta}^{-T} \cdot \boldsymbol{n}_{n}\right)\right) \hat{J}_{\Delta} \mathrm{d} \Gamma_{n} \\
\int_{\Omega_{n}} q \hat{J} \operatorname{tr}\left(\boldsymbol{H}_{\delta x}\right) \hat{J}_{\Delta} \mathrm{d} \Omega_{n}=-\int_{\Omega_{n}} q(\hat{J}-1) \hat{J}_{\Delta} \mathrm{d} \Omega_{n}
\end{array}\right.
$$

As most expressions are now written as the most recent estimate in $\hat{\Omega}$, it is convenient to also transform the integrals to the most recent estimated configuration $\hat{\Omega}$, by using $\hat{\nabla}=\hat{\boldsymbol{F}}_{\Delta}^{-T} \cdot \nabla_{n}$ and $\mathrm{d} \hat{\Omega}=\hat{J}_{\Delta} \mathrm{d} \Omega_{n}$ (see figure A.1).

$$
\left\{\begin{array}{c}
\int_{\hat{\Omega}}(\hat{\nabla} \boldsymbol{w})^{T}:\left[-\boldsymbol{H}_{\delta x} \cdot \hat{\boldsymbol{\sigma}}+\hat{\boldsymbol{\sigma}} \operatorname{tr}\left(\boldsymbol{H}_{\delta x}\right)+\delta \boldsymbol{\sigma}\right] \mathrm{d} \hat{\Omega}= \\
\int_{\hat{\Gamma}} \boldsymbol{w} \cdot \hat{\boldsymbol{\sigma}} \cdot \hat{\boldsymbol{n}} \mathrm{d} \hat{\Gamma}-\int_{\hat{\Omega}}(\hat{\nabla} \boldsymbol{w})^{T}: \hat{\boldsymbol{\sigma}} \mathrm{d} \hat{\Omega} \\
\int_{\hat{\Omega}} q \hat{J} \operatorname{tr}\left(\boldsymbol{H}_{\delta x}\right) \mathrm{d} \hat{\Omega}=-\int_{\hat{\Omega}} q(\hat{J}-1) \mathrm{d} \hat{\Omega}
\end{array}\right.
$$

\section{Error $\delta \boldsymbol{\sigma}$}

Only for $\delta \boldsymbol{\sigma}$ an expression needs to be derived. Linearization of $\boldsymbol{\sigma}$ yields:

$$
\begin{aligned}
\boldsymbol{\sigma} & =-p \boldsymbol{I}+\boldsymbol{\tau} \\
& =-\hat{p} \boldsymbol{I}+\hat{\boldsymbol{\tau}}-\delta p \boldsymbol{I}+\delta \boldsymbol{\tau} \\
& =\hat{\boldsymbol{\sigma}}+\delta \boldsymbol{\sigma} \\
\delta \boldsymbol{\sigma} & =-\delta p \boldsymbol{I}+\delta \boldsymbol{\tau}
\end{aligned}
$$

To describe the mechanical behavior of an arterial wall, various linear and nonlinear material models can be used. Here, the nonlinear hyperelastic material model from Raghavan and Vorp (2000) was used, which is a commonly used material model in abdominal aortic aneurysm wall mechanics. The extra stress $\tau$ was extended with an extra penalty parameter $\gamma$ to facilitate volume preservation in the momentum equation during the computations:

$$
\boldsymbol{\tau}=2\left(\alpha+2 \beta\left(I_{B}-3\right)\right)(\boldsymbol{B}-\boldsymbol{I})+2 \gamma\left(I I I_{B}-1\right) \boldsymbol{I}
$$


The parameters $\alpha, \beta$ and $\gamma$ are material parameters, $\boldsymbol{B}=\boldsymbol{F} \cdot \boldsymbol{F}^{T}$ is the left CauchyGreen deformation tensor and $I_{B}$ and $I I I_{B}$ are the first and third invariants of $\boldsymbol{B}$. Linearization of $\boldsymbol{B}=\hat{\boldsymbol{B}}+\delta \boldsymbol{B}, I_{B}{ }^{3}$ and $I I I_{B}{ }^{4}$ results in the following error term of the extra stress tensor:

$$
\delta \boldsymbol{\tau}=4 \beta \operatorname{tr}(\delta \boldsymbol{B})(\hat{\boldsymbol{B}}-\boldsymbol{I})+g_{1} \delta \boldsymbol{B}+2 \gamma I \hat{I} I_{B} \cdot\left(\hat{\boldsymbol{B}}^{-1}: \delta \boldsymbol{B}\right) \boldsymbol{I}
$$

with $g_{1}=2 \alpha+4 \beta\left(\hat{I}_{B}-3\right)$

To derive an expression for $\delta \boldsymbol{B}$ we use the definition $\boldsymbol{B}=\boldsymbol{F} \cdot \boldsymbol{F}^{T}$ and ignore the double error products:

$$
\begin{aligned}
\delta \boldsymbol{B} & =\delta \boldsymbol{F} \cdot \hat{\boldsymbol{F}}^{T}+\hat{\boldsymbol{F}} \cdot \delta \boldsymbol{F}^{T} \\
& =\left(\nabla_{0} \delta \boldsymbol{x}\right)^{T} \cdot \hat{\boldsymbol{F}}^{T}+\hat{\boldsymbol{F}} \cdot\left(\nabla_{0} \delta \boldsymbol{x}\right) \\
& =\left(\hat{\boldsymbol{F}}^{T} \cdot \hat{\nabla} \delta \boldsymbol{x}\right)^{T} \cdot \hat{\boldsymbol{F}}^{T}+\hat{\boldsymbol{F}} \cdot\left(\hat{\boldsymbol{F}}^{T} \cdot \hat{\nabla} \delta \boldsymbol{x}\right) \\
& =(\hat{\nabla} \delta \boldsymbol{x})^{T} \cdot \hat{\boldsymbol{F}} \cdot \hat{\boldsymbol{F}}^{T}+\hat{\boldsymbol{F}} \cdot \hat{\boldsymbol{F}}^{T} \cdot(\hat{\nabla} \delta \boldsymbol{x}) \\
& =\boldsymbol{H}_{\delta x} \cdot \hat{\boldsymbol{B}}+\hat{\boldsymbol{B}} \cdot \boldsymbol{H}_{\delta x}{ }^{T}
\end{aligned}
$$

Using this expression, it follows that:

$$
\operatorname{tr}(\delta \boldsymbol{B})=\left(\hat{\boldsymbol{B}}+\hat{\boldsymbol{B}}^{T}\right): \boldsymbol{H}_{\delta x}
$$

Now, $\delta \boldsymbol{B}$ and $\operatorname{tr}(\delta \boldsymbol{B})$ are substituted in equation A.18 and the components of $\delta \boldsymbol{\tau}$ are arranged such, that the terms are written as a left multiplication with $\boldsymbol{H}_{\delta x}$ :

$$
\delta \boldsymbol{\tau}=\boldsymbol{M}: \boldsymbol{H}_{\delta x}
$$

For other material models, the form of equation A.21 can in most cases be adapted by constructing $\boldsymbol{M}$ differently. Together with $-\delta p \boldsymbol{I}$, the error in the extra stress tensor $\delta \boldsymbol{\tau}$ now forms $\delta \boldsymbol{\sigma}$ (equation A.16). Substitution in equation A.15 yields the linearized weighted residual formulation of the momentum and mass equations:

$$
\left\{\begin{array}{c}
\int_{\hat{\Omega}}(\hat{\nabla} \boldsymbol{w})^{T}:\left[-\boldsymbol{H}_{\delta x} \cdot \hat{\boldsymbol{\sigma}}+\hat{\boldsymbol{\sigma}} \operatorname{tr}\left(\boldsymbol{H}_{\delta x}\right)-\delta p \boldsymbol{I}+\boldsymbol{M}: \boldsymbol{H}_{\delta x}\right] \mathrm{d} \hat{\Omega}= \\
\int_{\hat{\Gamma}} \boldsymbol{w} \cdot \hat{\boldsymbol{\sigma}} \cdot \hat{\boldsymbol{n}} \mathrm{d} \hat{\Gamma}-\int_{\hat{\Omega}}(\hat{\nabla} \boldsymbol{w})^{T}: \hat{\boldsymbol{\sigma}} \mathrm{d} \hat{\Omega} \\
\int_{\hat{\Omega}} q \hat{J} \operatorname{tr}\left(\boldsymbol{H}_{\delta x}\right) \mathrm{d} \hat{\Omega}=-\int_{\hat{\Omega}} q(\hat{J}-1) \mathrm{d} \hat{\Omega}
\end{array}\right.
$$

\footnotetext{
${ }^{3}$ Use $I_{B}=\operatorname{tr}(\mathrm{B})=\operatorname{tr}(\hat{\mathrm{B}}+\delta \mathrm{B})=\operatorname{tr}(\hat{\mathrm{B}})+\operatorname{tr}(\delta \mathrm{B})=\hat{I}_{B}+\operatorname{tr}(\delta \mathrm{B})$

${ }^{4} \mathrm{Use} I I I_{B}=\operatorname{det}(\hat{\boldsymbol{B}}+\delta \boldsymbol{B})=\operatorname{det}\left(\hat{\boldsymbol{B}} \cdot\left(\boldsymbol{I}+\hat{\boldsymbol{B}}^{-1} \cdot \delta \boldsymbol{B}\right)\right) \approx \operatorname{det}(\hat{\boldsymbol{B}}) \cdot\left(1+\hat{\boldsymbol{B}}^{-1}: \delta \boldsymbol{B}\right)=$ $I \hat{I} I_{B}+I \hat{I} I_{B} \cdot\left(\hat{\boldsymbol{B}}^{-1}: \delta \boldsymbol{B}\right)$
} 


\section{Matrix Notation}

We will now transform the coordinate free tensor formulation to a matrix formulation with respect to a Cartesian basis in $3 \mathrm{D}$ space. First, we store the components of the tensor $(\hat{\nabla} \boldsymbol{w})$ in a column $\underset{\sim}{H}$, as is common in the finite element package Sepran (Sepra, Delft, the Netherlands):

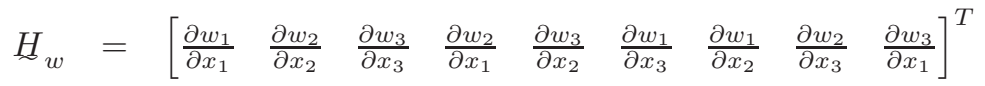

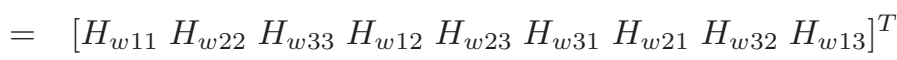

Similarly, the components of $(\hat{\nabla} \delta \boldsymbol{x})$ can be stored in ${\underset{\sim}{H}}_{\delta x}$. The double dot products in equations A.22 can be evaluated as:

$$
(\hat{\nabla} \boldsymbol{w})^{T}: \boldsymbol{A}=\boldsymbol{H}_{w}: \boldsymbol{A}={\underset{\sim}{H}}_{w}^{T} \underset{\sim}{A}
$$

which defines how the components of matrix $\boldsymbol{A}$ are stored in column $\underset{\sim}{A}$ :

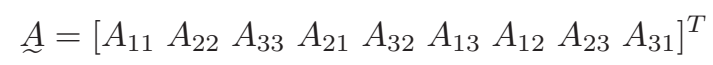

Application to all components in equations A.22 yields:

$$
\begin{aligned}
& \boldsymbol{H}_{w}:\left[-\boldsymbol{H}_{\delta x} \cdot \hat{\boldsymbol{\sigma}}\right]=H_{w}^{T} \underline{D}_{F} \underline{H}_{\delta x} \\
& \boldsymbol{H}_{w}:\left[\hat{\boldsymbol{\sigma}} \operatorname{tr}\left(\boldsymbol{H}_{\delta x}\right)\right]=\hat{H}_{w}^{T} \underline{D}_{J} \underline{H}_{\delta x} \\
& \boldsymbol{H}_{w}: \quad\left[\boldsymbol{M}: \boldsymbol{H}_{\delta x}\right]=H_{w}^{T} \underline{D}_{T} H_{\delta x}
\end{aligned}
$$

In combination with equations A.23 and A.24, this defines how $\hat{\boldsymbol{\sigma}}$ and $\boldsymbol{M}$ are stored in $\underline{D}_{F}, \underline{D}_{J}$ and $\underline{D}_{T}$. Summarizing, equations A.22 can now be written as:

$$
\left\{\begin{aligned}
\int_{\hat{\Omega}} H_{w}^{T}\left[\underline{D}_{F}+\underline{D}_{J}+\right. & \left.\underline{D}_{T}\right] H_{\delta x} \mathrm{~d} \hat{\Omega}-\int_{\hat{\Omega}} \delta p \hat{\nabla} \cdot \boldsymbol{w} \mathrm{d} \hat{\Omega}= \\
& -\int_{\hat{\Omega}} H_{w}^{T} \mathrm{~d} \mathrm{~d} \hat{\Omega}+\int_{\hat{\Gamma}} \boldsymbol{w} \cdot \hat{\boldsymbol{\sigma}} \cdot \hat{\boldsymbol{n}} \mathrm{d} \hat{\Gamma} \\
\int_{\hat{\Omega}} q \hat{J}(\hat{\nabla} \cdot \delta \boldsymbol{x}) \mathrm{d} \hat{\Omega}= & -\int_{\hat{\Omega}} q(\hat{J}-1) \mathrm{d} \hat{\Omega}
\end{aligned}\right.
$$

\section{Discretization}

To obtain a discrete set of finite element equations, the domain $\Omega$ is subdivided into nonoverlapping sub domains $\Omega^{e}$, called elements.The position field contains three components $\left(x_{1}, x_{2}, x_{3}\right)$. Within each element, the components of the position field $x_{j}^{e}$ (with $j=1,2,3$ ) and the pressure field $p^{e}$ are approximated as interpolations between values at a limited number of nodal points:

$$
\begin{aligned}
& x_{j}^{e}(\boldsymbol{x}, t)=\sum_{i=1}^{n_{x}} \Phi^{i}(\boldsymbol{x}) x_{j}^{i}(t)=\Phi^{T} x_{j}^{e} \\
& p^{e}(\boldsymbol{x}, t)=\sum_{i=1}^{n_{p}} \Psi^{i}(\boldsymbol{x}) p^{i}(t)=\Psi^{T} \underline{p}^{e}
\end{aligned}
$$


Here, $n_{x}$ and $n_{p}$ are the number nodes for the position and pressure field, respectively. $\underset{\Phi}{\Phi}=\left[\Phi^{1}, \ldots, \Phi^{n_{x}}\right]^{T}$ and $\underset{\Psi}{\Psi}=\left[\Psi^{1}, \ldots, \Psi^{n_{p}}\right]^{T}$ are the columns of the interpolation shape functions for the position and pressure and $\underset{\sim}{x}=\left[\boldsymbol{x}^{1}, \ldots, \boldsymbol{x}^{n_{x}}\right]$ and ${\underset{\sim}{p}}^{e}=\left[p^{1}, \ldots, p^{n_{p}}\right]$ are the columns with the nodal positions and pressures. The weighting functions $\boldsymbol{w}$ containing the three components $\left(w_{1}, w_{2}, w_{3}\right)$ and $q$ can also be defined on element level:

$$
\begin{aligned}
& w_{j}^{e}(\boldsymbol{x}, t)=\sum_{i=1}^{n_{x}} \Phi^{i}(\boldsymbol{x}) w_{j}^{i}(t)=\Phi^{T} w_{j}^{e} \quad(j=1,2,3) \\
& q^{e}(\boldsymbol{x}, t)=\sum_{i=1}^{n_{p}} \Psi^{i}(\boldsymbol{x}) q^{i}(t)=\Psi^{T} q^{e}
\end{aligned}
$$

Based on these discretizations, we can now reconsider the column $\underset{\sim}{H}$, as was defined in equation A.23:

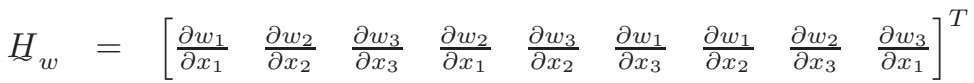

$$
\begin{aligned}
& =\left[\frac{\partial \Phi^{T} w_{1}^{e}}{\partial x_{1}} \frac{\partial \Phi^{T} w_{2}^{e}}{\partial x_{2}} \frac{\partial \Phi^{T} w_{3}^{e}}{\partial x_{3}} \frac{\partial \Phi^{T} w_{2}^{e}}{\partial x_{1}} \frac{\partial \Phi^{T} w_{3}^{e}}{\partial x_{2}} \frac{\partial \Phi^{T} w_{\mathcal{\sim}}^{e}}{\partial x_{3}} \frac{\partial \Phi^{T} w_{1}^{e}}{\partial x_{2}} \frac{\partial \Phi^{T} w_{2}^{e}}{\partial x_{3}} \frac{\partial \Phi^{T} w_{3}^{e}}{\partial x_{1}}\right]^{T} \\
& =\left[\begin{array}{llllllllll}
\frac{\partial \Phi^{1}}{\partial x_{1}} & 0 & 0 & \frac{\partial \Phi^{2}}{\partial x_{1}} & 0 & 0 & \ldots & \frac{\partial \Phi^{n_{x}}}{\partial x_{1}} & 0 & 0 \\
0 & \frac{\partial \Phi^{1}}{\partial x_{2}} & 0 & 0 & \frac{\partial \Phi^{2}}{\partial x_{2}} & 0 & \ldots & 0 & \frac{\partial \Phi^{n_{x}}}{\partial x_{2}} & 0 \\
0 & 0 & \frac{\partial \Phi^{1}}{\partial x_{3}} & 0 & 0 & \frac{\partial \Phi^{2}}{\partial x_{3}} & \ldots & 0 & 0 & \frac{\partial \Phi^{n_{x}}}{\partial x_{3}} \\
0 & \frac{\partial \Phi^{1}}{\partial x_{1}} & 0 & 0 & \frac{\partial \Phi^{2}}{\partial x_{1}} & 0 & \ldots & 0 & \frac{\partial \Phi^{n_{x}}}{\partial x_{1}} & 0 \\
0 & 0 & \frac{\partial \Phi^{1}}{\partial x_{2}} & 0 & 0 & \frac{\partial \Phi^{2}}{\partial x_{2}} & \ldots & 0 & 0 & \frac{\partial \Phi^{n_{x}}}{\partial x_{2}} \\
\frac{\partial \Phi^{1}}{\partial x_{3}} & 0 & 0 & \frac{\partial \Phi^{2}}{\partial x_{3}} & 0 & 0 & \ldots & \frac{\partial \Phi^{n_{x}}}{\partial x_{3}} & 0 & 0 \\
\frac{\partial \Phi^{1}}{\partial x_{2}} & 0 & 0 & \frac{\partial \Phi^{2}}{\partial x_{2}} & 0 & 0 & \ldots & \frac{\partial \Phi^{n_{x}}}{\partial x_{2}} & 0 & 0 \\
0 & \frac{\partial \Phi^{1}}{\partial x_{3}} & 0 & 0 & \frac{\partial \Phi^{2}}{\partial x_{3}} & 0 & \ldots & 0 & \frac{\partial \Phi^{n_{x}}}{\partial x_{3}} & 0 \\
0 & 0 & \frac{\partial \Phi^{1}}{\partial x_{1}} & 0 & 0 & \frac{\partial \Phi^{2}}{\partial x_{1}} & \ldots & 0 & 0 & \frac{\partial \Phi^{n_{x}}}{\partial x_{1}}
\end{array}\right]\left[\begin{array}{l}
w_{1}^{1} \\
w_{2}^{1} \\
w_{3}^{1} \\
w_{1}^{2} \\
w_{2}^{2} \\
w_{3}^{2} \\
\vdots \\
w_{1}^{n_{x}} \\
w_{2}^{n_{x}} \\
w_{3}^{n_{x}}
\end{array}\right] \\
& =\underline{B}_{\Phi} w^{e}
\end{aligned}
$$

similar, for $\underset{\sim}{H} \delta x$ yields:

$$
\underline{\sim}_{\delta x}=\underline{B}_{\Phi} \delta \underbrace{e}
$$

For $\hat{\nabla} \cdot \boldsymbol{w}$ can be substituted:

$$
\begin{aligned}
\hat{\nabla} \cdot \boldsymbol{w} & =\frac{\partial w_{1}}{\partial x_{1}}+\frac{\partial w_{2}}{\partial x_{2}}+\frac{\partial w_{3}}{\partial x_{3}} \\
& =\frac{\partial \Phi^{\underline{w}} w_{1}^{e}}{\partial x_{1}}+\frac{\partial \Phi^{e} w_{2}^{e}}{\partial x_{2}}+\frac{\partial \Phi^{e} w_{3}^{e}}{\partial x_{3}} \\
& =\left[\frac{\partial \Phi^{1}}{\partial x_{1}} \frac{\partial \Phi^{1}}{\partial x_{2}} \frac{\partial \Phi^{1}}{\partial x_{3}} \frac{\partial \Phi^{2}}{\partial x_{1}} \frac{\partial \Phi^{2}}{\partial x_{2}} \frac{\partial \Phi^{3}}{\partial x_{3}} \ldots \frac{\partial \Phi^{n_{x}}}{\partial x_{1}} \frac{\partial \Phi^{n_{x}}}{\partial x_{2}} \frac{\partial \Phi^{n_{x}}}{\partial x_{3}}\right] w^{e} \\
& =b_{\Phi}^{T} w^{e}
\end{aligned}
$$


Similar:

$$
\hat{\nabla} \cdot \delta \boldsymbol{x}=\underline{b}_{\Phi}^{T} \delta \underline{\sim}^{e}
$$

Finally, we elaborate the integrand of the surface integral as:

$$
\begin{aligned}
\boldsymbol{w} \cdot \hat{\boldsymbol{\sigma}} \cdot \hat{\boldsymbol{n}} & =\boldsymbol{w} \cdot \boldsymbol{s} \\
& =w_{1} s_{1}+w_{2} s_{2}+w_{3} s_{3} \\
& =\left(\Phi w^{e}\right)^{T} \Phi s^{e}
\end{aligned}
$$

Substitution in equations A.27 yields:

$$
\left\{\begin{array}{c}
\left.\sum_{1}^{n_{e l}}\left(\int_{\Omega^{e}} \underline{B}_{\Phi} w^{e}\right)^{T}\left[\underline{D}_{F}+\underline{D}_{J}+\underline{D}_{T}\right] \underline{B}_{\Phi} \delta x^{e} \mathrm{~d} \Omega^{e}-\int_{\Omega^{e}} \Psi^{T} \delta \underline{p}^{e} b_{\Phi}^{T} w^{e} \mathrm{~d} \Omega^{e}\right)= \\
\left.\sum_{1}^{n_{e l}}\left(-\int_{\Omega^{e}}\left(\underline{B}_{\Phi} w^{e}\right)^{T} \varnothing \mathrm{d} \Omega^{e}\right)+\int_{\Gamma^{e}}\left(\Phi w^{e}\right)^{T} \Phi s^{e} \mathrm{~d} \Gamma^{e}\right) \\
\sum_{1}^{n_{e l}}\left(\int_{\Omega^{e}} \Psi^{T} q^{e} b_{\Phi}^{T} \delta x^{e} \mathrm{~d} \Omega^{e}\right)=\sum_{1}^{n_{e l}}\left(-\int_{\Omega^{e}} \Psi^{T} q^{e} \frac{J-1}{J} \mathrm{~d} \Omega^{e}\right)
\end{array}\right.
$$

The second equation was divided by $J$ to obtain a symmetric stiffness matrix. As $\underline{w}^{e}$, $\delta \underline{x}^{e}, \delta \underline{p}^{e}$, and $q^{e}$ are arrays with constants, they can be taken out of the integrals:

$$
\left\{\begin{array}{l}
\sum_{1}^{n_{e l}}\left(\left(w^{e}\right)^{T} \underline{K}^{e} \delta x^{e}-\left(w^{e}\right)^{T}\left(\underline{P}^{e}\right)^{T} \delta \underline{p}^{e}\right)=\sum_{1}^{n_{e l}}\left(\left(w^{e}\right)^{T} x_{x}^{e}\right) \\
\sum_{1}^{n_{e l}}\left(\left(q^{e}\right)^{T} \underline{P}^{e} \delta x^{e}\right)=\sum_{1}^{n_{e l}}\left(-\left(q^{e}\right)^{T} x_{p}^{e}\right)
\end{array}\right.
$$

with:

$$
\begin{aligned}
\underline{K}^{e} & =\int_{\Omega^{e}}\left(\underline{B}_{\Phi}\right)^{T}\left[\underline{D}_{F}+\underline{D}_{J}+\underline{D}_{T}\right] \underline{B}_{\Phi} \mathrm{d} \Omega^{e} \\
\underline{P}^{e} & =\int_{\Omega^{e}} \underline{b}_{\Phi}^{T} \underline{\Psi} \mathrm{d} \Omega^{e} \\
\underline{r}_{x}^{e} & =\int_{\Omega^{e}}\left(\underline{B}_{\Phi}\right)^{T} \sigma \mathrm{d} \Omega^{e}+\int_{\Gamma^{e}} \underline{\Phi}^{T} \underline{\Phi}_{\sim} s_{e} \mathrm{~d} \Gamma^{e} \\
\underline{\sim}_{p}^{e} & =\int_{\Omega^{e}} \underset{\Psi}{\Psi} \frac{J-1}{J} \mathrm{~d} \Omega^{e}
\end{aligned}
$$

Now all element columns and matrices can be assembled into columns and matrices at the global level:

$$
\left\{\begin{array}{l}
w^{T}\left(\underline{K} \delta x-\underline{P}^{T} \delta \underline{p}\right)=w^{T} x_{x} \\
\underline{q}^{T} \underline{P} \delta x=q^{T} x_{p}
\end{array}\right.
$$


which should hold for all weighting functions $\underset{\sim}{w}$ and $\underset{\sim}{q}$. Therefore, this formulation is equivalent to:

$$
\left[\begin{array}{cc}
\underline{K} & \underline{P}^{T} \\
\underline{P} & \underline{0}
\end{array}\right]\left[\begin{array}{l}
\delta x \\
\delta p
\end{array}\right]=\left[\begin{array}{l}
x_{x} \\
x_{p}
\end{array}\right]
$$

This final set of equations is used to solve each pressure step (increment) in a iterative fashion. Thereto, the initial estimates $\hat{\boldsymbol{x}}_{i}$ and $\hat{p}_{i}$ are used to compute the matrices $\underline{K}$ and $\underline{P}$ and the tensors $\underline{\sim}_{x}$ and $\underline{r}_{p}$. The error terms $\delta \underset{\sim}{x}$ and $\delta \underset{\sim}{p}$ are determined and the solution is updated with $\hat{\boldsymbol{x}}_{i+1}=\hat{\boldsymbol{x}}_{i}+\delta \underset{\sim}{x}$ and $\hat{p}_{i+1}=\hat{p}_{i}+\delta p$. A converged solution has been reached when either an absolute $\left([\|\underset{\sim}{\delta}\|\|\delta \underset{\sim}{p}\|]^{T}<\epsilon\right)$ or relative

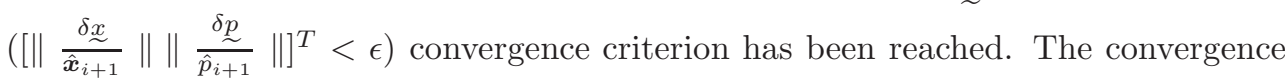
criterion $\epsilon$ in our simulations was chosen to be relative and was set at 0.001 . When the criterion has not been reached, $\left[\hat{\boldsymbol{x}}_{i+1} \hat{p}_{i+1}\right]^{T}$ will be used as new initial estimate. When the solution is indeed converged, the next pressure increment can start.

Convergence of the iterative scheme may only be achieved if the external load is applied in sufficiently small steps. At each step, the non-linear system is linearized with Newton's method as described. As the resulting matrices are very large, no direct computation can be performed to determine the error terms $\delta x$ and $\delta p$. Therefore, an incomplete LU decomposition matrix pre-conditioner is used for the linear system and a Bi-CGSTAB method to solve the system.

\section{Backward Incremental Method}

In the previous sections, we have derived the complete finite element formulation of the balance laws of mass and momentum and is described how the resulting set of equations is iteratively solved within each pressure increment. In general, the pressure is increased stepwise and each step, the iteration process will lead to a new configuration and stress state. In general the complete procedure summarizes to:

$$
\left[\boldsymbol{F}_{\Delta}, \delta \boldsymbol{\sigma}\right]=f\left(\Omega_{n-1}, \boldsymbol{F}_{n-1}, \boldsymbol{\sigma}_{n-1}, p_{\Delta}, G\right)
$$

The updates in the deformation $\boldsymbol{F}_{\Delta}$ and stress $\delta \boldsymbol{\sigma}$ at each pressure increment thus is a function of the previous configuration $\Omega_{n-1}$, the accumulated deformation gradient tensor $\boldsymbol{F}_{n-1}$, the accumulated stress $\boldsymbol{\sigma}_{n-1}$ the pressure step $p_{\Delta}$, and the set of material properties $G$.

The backward incremental method accounts for the initial stress and initial geometry by solving the system of equations in two incremental pressure stages. Stage 1 is the actual backward incremental stage and runs from $0 \mathrm{kPa}$ to the patient specific mean arterial pressure. In this stage, the initial stress is determined that corresponds with the initial geometry. 
Stage 2 is performed conventionally and runs from the mean arterial pressure to a systolic pressure of $18.7 \mathrm{kPa}(140 \mathrm{mmHg})$. For both stages, 16 incremental pressure steps were used to apply the pressure starting from the reference geometry $\Omega_{r}$ :
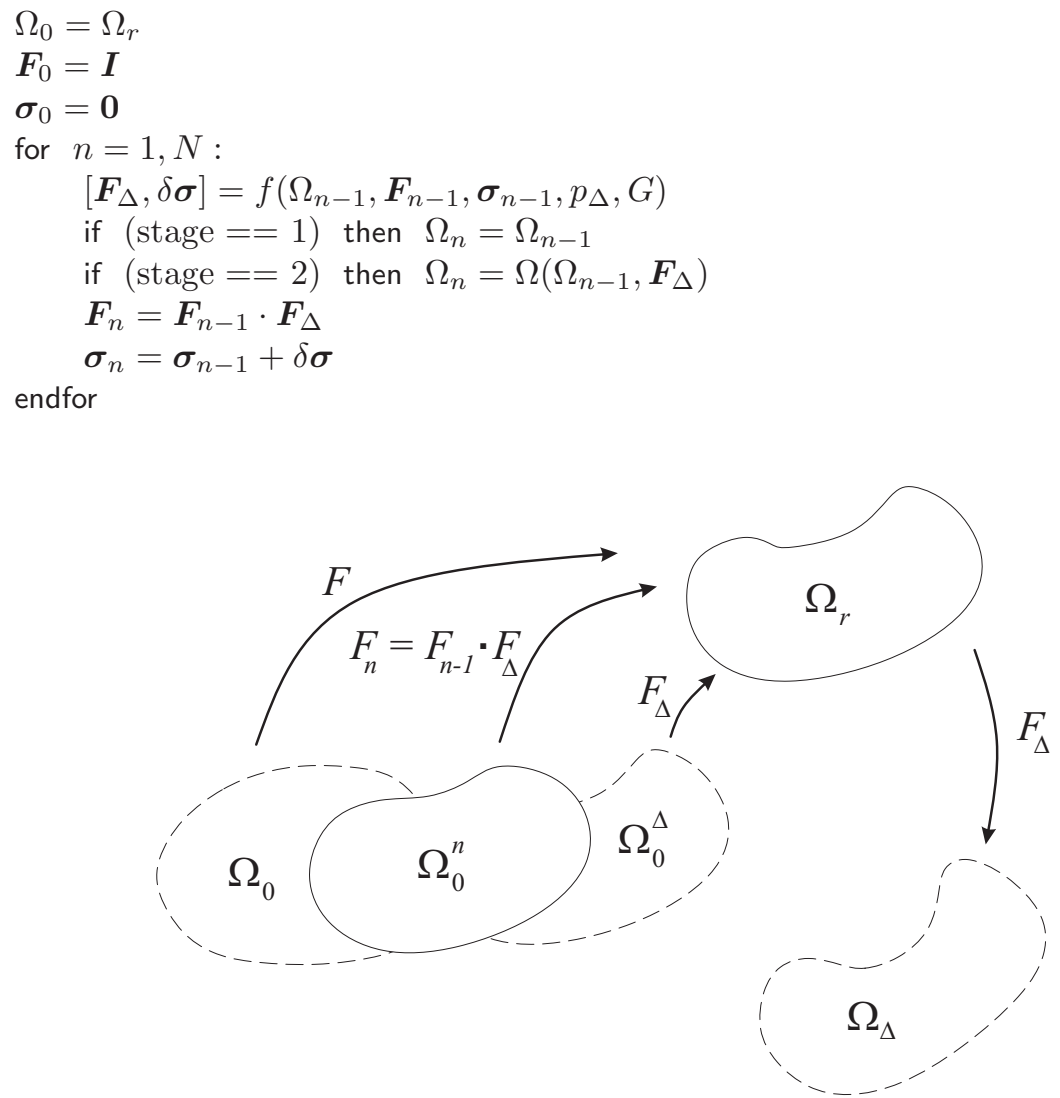

Figure A.2: The backward incremental method. By computing the forward deformation from $\Omega_{r}$ to $\Omega_{\Delta}$ in step $n$, the initial geometry $\Omega_{0}^{n}$ is implicitly updated, until $\Omega_{0}$ has been reached. This initial geometry is load free and stress free.

In stage 1 (the backward incremental stage), the geometry is not updated with the computed deformation tensor $\boldsymbol{F}_{\Delta}$. Therefore, an imbalance exists at the beginning of each increment. To ensure that at the end of stage 1 the system is balanced in terms of displacements and pressures, the final pressure step is chosen extremely small. Implicitly, in stage $1, \boldsymbol{F}_{n}=\boldsymbol{F}_{n-1} \cdot \boldsymbol{F}_{\Delta}$ updates the load free configuration and $\boldsymbol{\sigma}(t)=\boldsymbol{\sigma}_{n-1}+\delta \boldsymbol{\sigma}$ updates the initial stress (see figure A.2). Once in stage 1 equilibrium has been reached, the convexity of the constitutive equations ensures a unique initial stress state at the reference configuration $\Omega_{r}$ and a unique load free and stress free configuration $\Omega_{0}$. 


\section{References}

R. Adolph, D. A. Vorp, D. L. Steed, M. W. Webster, M. V. Kameneva, and S. C. Watkins. Cellular content and permeability of intraluminal thrombus in abdominal aortic aneurysm. J. Vasc. Surg., 25(5):916-926, 1997.

V. Alastrué, E. Peña, M. A. Martínez, and M. Doblaré. Assessing the use of the "opening angle method" to enforce residual stresses in patient-specific arteries. Ann. Biomed. Eng., 35(10):1821-1837, 2007.

J. H. Ashton, J. P. vande Geest, B. R. Simon, and D. G. Haskett. Compressive mechanical properties of the intraluminal thrombus in abdominal aortic aneurysms and fibrin-based thrombus mimics. J. Biomech., 42(3):197-201, 2009.

P. S. Basnyat, A. H. Biffin, L. G. Moseley, A. R. Hedges, and M. H. Lewis. Mortality from ruptured abdominal aortic aneurysm in Wales. Br. J. Surg., 86(6):765-770, 1999.

F. Boschetti, E. di Martino, and G. Gioda. A poroviscoelastic model of intraluminal thrombus from abdominal aortic aneurysms. Proceedings of the 2007 Summer Bioengineering Conference, Keystone CO, 2007.

M. J. Bown, A. J. Sutton, P. R. Bell, and R. D. Sayers. A meta-analysis of 50 years of ruptured abdominal aortic aneurysm repair. Br. J. Surg., 89(6):714-730, 2002.

M. Breeuwer, S. de Putter, U. Kose, L. Speelman, K. Visser, F. Gerritsen, R. Hoogeveen, R. Krams, H. van den Bosch, J. Buth, T. Gunther, B. Wolters, E. van Dam, and F.N. van de Vosse. Towards patient-specific risk assessment of abdominal aortic aneurysm. Med. Biol. Eng. Comput., 46(11):1085-1095, 2008.

J. S. Budd, D. R. Finch, and P. G. Carter. A study of the mortality from ruptured abdominal aortic aneurysms in a district community. Eur. J. Vasc. Surg., 3(4): 351-354, 1989.

R. L. Bush, P. H. Lin, and A. B. Lumsden. Endovascular management of abdominal aortic aneurysms. J. Cardiovasc. Surg. (Torino), 44(4):527-534, 2003.

J. S. Campa, R. M. Greenhalgh, and J. T. Powell. Elastin degradation in abdominal aortic aneurysms. Atherosclerosis, 65(1-2):13-21, 1987. 
E. L. Chaikof, M. F. Fillinger, J. S. Matsumura, R. B. Rutherford, G. H. White, J. D. Blankensteijn, V. M. Bernhard, P. L. Harris, K. C. Kent, J. May, F. J. Veith, and C. K. Zarins. Identifying and grading factors that modify the outcome of endovascular aortic aneurysm repair. J. Vasc. Surg., 35(5):1061-1066, 2002.

E. Choke, G. Cockerill, W. R. Wilson, S. Sayed, J. Dawson, I. Loftus, and M. M. Thompson. A review of biological factors implicated in abdominal aortic aneurysm rupture. Eur. J. Vasc. Endovasc. Surg., 30(3):227-244, 2005.

E. S. Crawford and E. S. Cohen. Aortic aneurysm: a multifocal disease. Arch. Surg., 117(11):1393-1400, 1982

R. C. Darling, C. R. Messina, D. C. Brewster, and L. W. Ottinger. Autopsy study of unoperated abdominal aortic aneurysms. The case for early resection. Circulation, 56(3 Suppl):II161-II164, 1977.

A. Daugherty and L. A. Cassis. Mechanisms of abdominal aortic aneurysm formation. Curr. Atheroscler. Rep., 4(3):222-227, 2002.

S. de Putter, M. Breeuwer, U. Kose, F. Laffargue, J.-M. Rouet, R. Hoogeveen, H. van de Bosch, J. Buth, F. N. van de Vosse, and F. A. Gerritsen. Automatic determination of the dynamic geometry of abdominal aortic aneurysm from MR with application to wall stress simulations. Proceedings of the 19th International Computer Assisted Radiology and Surgery Conference, pages 339-344, 2005.

S. de Putter, M. Breeuwer, F. N. van de Vosse, U Kose, and F. A. Gerritsen. Patientspecific models of wall stress in abdominal aortic aneurysm: a comparison between MR and CT. Progress in biomedical optics and imaging, 7(1), 2006a.

S. de Putter, F. N. van de Vosse, M. Breeuwer, and F. A. Gerritsen. Local influence of calcifications on the wall mechanics of abdominal aortic aneurysm. Progress in biomedical optics and imaging, 7(2), 2006b.

S. de Putter, B. J. Wolters, M. C. Rutten, M. Breeuwer, F. A. Gerritsen, and F. N. van de Vosse. Patient-specific initial wall stress in abdominal aortic aneurysms with a backward incremental method. J. Biomech., 40(5):1081-1090, 2007.

H. Delingette. Modélisation, Déformation et Reconnaissance d'objets tridimensionnels a l'aide de maillages simplexes. PhD thesis, Ecole Centrale de Paris, 1994.

E. S. di Martino and D. A. Vorp. Effect of variation in intraluminal thrombus constitutive properties on abdominal aortic aneurysm wall stress. Ann. Biomed. Eng., 31(7):804-809, 2003.

E. S. di Martino, S. Mantero, F. Inzoli, G. Melissano, D. Astore, R. Chiesa, and R. Fumero. Biomechanics of abdominal aortic aneurysm in the presence of endoluminal thrombus: experimental characterisation and structural static computational analysis. Eur. J. Vasc. Endovasc. Surg., 15(4):290-299, 1998. 
H. Domanovits, M. Schillinger, M. Mullner, T. Holzenbein, K. Janata, K. Bayegan, and A. N. Laggner. Acute phase reactants in patients with abdominal aortic aneurysm. Atherosclerosis, 163(2):297-302, 2002.

B. J. Doyle, A. Callanan, and T. M. McGloughlin. A comparison of modelling techniques for computing wall stress in abdominal aortic aneurysms. Biomed. Eng. Online, 6:38, 2007.

G. Engstrom, G. Borner, B. Lindblad, L. Janzon, and F. Lindgarde. Incidence of fatal or repaired abdominal aortic aneurysm in relation to inflammation-sensitive plasma proteins. Arterioscler. Thromb. Vasc. Biol., 24(2):337-341, 2004.

M. F. Fillinger, M. L. Raghavan, S. P. Marra, J. L. Cronenwett, and F. E. Kennedy. In vivo analysis of mechanical wall stress and abdominal aortic aneurysm rupture risk. J. Vasc. Surg., 36(3):589-597, 2002.

M. F. Fillinger, S. P. Marra, M. L. Raghavan, and F. E. Kennedy. Prediction of rupture risk in abdominal aortic aneurysm during observation: wall stress versus diameter. J. Vasc. Surg., 37(4):724-732, 2003.

M. F. Fillinger, J. Racusin, R. K. Baker, J. L. Cronenwett, A. Teutelink, M. L. Schermerhorn, R. M. Zwolak, R. J. Powell, D. B. Walsh, and E. M. Rzucidlo. Anatomic characteristics of ruptured abdominal aortic aneurysm on conventional CT scans: Implications for rupture risk. J. Vasc. Surg., 39(6):1243-1252, 2004.

M. D. Gasbarro, E. S. di Martino, C. M. Scotti, E. A. Finol, and K. Shimada. Computational modeling of abdominal aortic aneurysms: A new simulation technique that demonstrates the importance of including realistic fluid motion, spinal column, and internal organs. Proceedings of the 2005 Summer Bioengineering Conference, Vail CO, 2005.

T. C. Gasser, G. Görgülü, M. Folkesson, and J. Swedenborg. Failure properties of intraluminal thrombus in abdominal aortic aneurysm under static and pulsating mechanical loads. J. Vasc. Surg., 48(1):179-188, 2008.

O. Gérard, A. Collet Billon, J.-M. Rouet, M. Jacob, M. Fradkin, and C. Allouche. Efficient model-based quantification of left ventricular function in 3-d echocardiography. IEEE Transactions on Medical Imaging, (21):1059-1068, 2002 .

J. Golledge, P. S. Tsao, R. L. Dalman, and P. E. Norman. Circulating markers of abdominal aortic aneurysm presence and progression. Circulation, 118(23):2382$2392,2008$.

T. J. Gorham, J. Taylor, and S. Raptis. Endovascular treatment of abdominal aortic aneurysm. Br. J. Surg., 91(7):815-827, 2004. 
R. M. Greenhalgh, J. F. Forbes, F. G. Fowkes, J. T. Powel, C. V. Ruckley, A. R. Brady, L. C. Brown, and S. G. Thompson. Early elective open surgical repair of small abdominal aortic aneurysms is not recommended: results of the UK small aneurysm trial. Eur. J. Vasc. Endovasc. Surg., 16(6):462-464, 1998.

R. M. Greenhalgh, L. C. Brown, G. P. Kwong, J. T. Powell, and S. G. Thompson. Comparison of endovascular aneurysm repair with open repair in patients with abdominal aortic aneurysm (EVAR trial 1), 30-day operative mortality results: randomised controlled trial. Lancet, 364(9437):843-848, 2004.

A. J. Hall, E. F. Busse, D. J. McCarville, and J. J. Burgess. Aortic wall tension as a predictive factor for abdominal aortic aneurysm rupture: improving the selection of patients for abdominal aortic aneurysm repair. Ann. Vasc. Surg., 14(2):152-157, 2000 .

S. S. Hans, O. Jareunpoon, M. Balasubramaniam, and G. B. Zelenock. Size and location of thrombus in intact and ruptured abdominal aortic aneurysms. J. Vasc. Surg., 41(4):584-588, 2005.

L. P. Harter, B. H. Gross, P. W. Callen, and R. A. Barth. Ultrasonic evaluation of abdominal aortic thrombus. J. Ultrasound Med., 1(8):315-318, 1982.

F. A. Hellenthal, I. L. Geenen, J. A. Teijink, S. Heeneman, and G. W. Schurink. Histological features of human abdominal aortic aneurysm are not related to clinical characteristics. Cardiovasc. Pathol., 2008.

M. S. Heng, M. J. Fagan, J. W. Collier, G. Desai, P. T. McCollum, and I. C. Chetter. Peak wall stress measurement in elective and acute abdominal aortic aneurysms. J. Vasc. Surg., 47(1):17-22, 2008.

J. W. Hinnen, D. J. Rixen, O. H. Koning, J. H. van Bockel, and J. F. Hamming. Development of fibrinous thrombus analogue for in-vitro abdominal aortic aneurysm studies. J. Biomech., 40(2):289-295, 2007.

G. A. Holzapfel, G. Sommer, and P. Regitnig. Anisotropic mechanical properties of tissue components in human atherosclerotic plaques. J. Biomech. Eng., 126(5): 657-665, 2004.

J. Hua and W. R. Mower. Simple geometric characteristics fail to reliably predict abdominal aortic aneurysm wall stresses. J. Vasc. Surg., 34(2):308-315, 2001.

G. C. Hunter, S. C. Leong, G. S. Yu, K. E. McIntyre, and V. M. Bernhard. Aortic blebs: possible site of aneurysm rupture. J. Vasc. Surg., 10(1):93-99, 1989.

K. W. Johnston. Ruptured abdominal aortic aneurysm: six-year follow-up results of a multicenter prospective study. J. Vasc. Surg., 19(5):888-900, 1994. 
K. W. Johnston, R. B. Rutherford, M. D. Tilson, D. M. Shah, L. Hollier, and J. C. Stanley. Suggested standards for reporting on arterial aneurysms. J. Vasc. Surg., 13(3):452-458, 1991.

L. G. Kim, P. S. Ra, H. A. Ashton, and S. G. Thompson. A sustained mortality benefit from screening for abdominal aortic aneurysm. Ann. Intern. Med., 146 (10):699-706, 2007.

H. Kurvers, F. J. Veith, E. C. Lipsitz, T. Ohki, N. J. Gargiulo, N. S. Cayne, W. D. Suggs, C. H. Timaran, G. Y. Kwon, S. J. Rhee, and C. Santiago. Discontinuous, staccato growth of abdominal aortic aneurysms. J. Am. Coll. Surg., 199(5):709$715,2004$.

F. A. Lederle, G. R. Johnson, S. E. Wilson, E. P. Chute, F. N. Littooy, D. Bandyk, W. C. Krupski, G. W. Barone, C. W. Acher, and D. J. Ballard. Prevalence and associations of abdominal aortic aneurysm detected through screening. Ann. Intern. Med., 126(6):441-449, 1997.

F. A. Lederle, G. R. Johnson, S. E. Wilson, D. J. Ballard, Jr. W. D. Jordan, J. Blebea, F. N. Littooy, J. A. Freischlag, D. Bandyk, J. H. Rapp, and A. A. Salam. Rupture rate of large abdominal aortic aneurysms in patients refusing or unfit for elective repair. JAMA, 287(22):2968-2972, 2002.

Z. Y. Li, J. King-Im, T. Y. Tang, E. Soh, T. C. See, and J. H. Gillard. Impact of calcification and intraluminal thrombus on the computed wall stresses of abdominal aortic aneurysm. J. Vasc. Surg., 47(5):928-935, 2008.

J. S. Lindholt, S. Vammen, H. Fasting, E. W. Henneberg, and L. Heickendorff. The plasma level of matrix metalloproteinase 9 may predict the natural history of small abdominal aortic aneurysms. A preliminary study. Eur. J. Vasc. Endovasc. Surg., 20(3):281-285, 2000.

A. Lopez-Candales, D. R. Holmes, S. Liao, M. J. Scott, S. A. Wickline, and R. W. Thompson. Decreased vascular smooth muscle cell density in medial degeneration of human abdominal aortic aneurysms. Am. J. Pathol., 150(3):993-1007, 1997.

H. M. Loree, A. J. Grodzinsky, S. Y. Park, L. J. Gibson, and R. T. Lee. Static circumferential tangential modulus of human atherosclerotic tissue. J. Biomech., 27(2):195-204, 1994.

R. E. Lovegrove, M. Javid, T. R. Magee, and R. B. Galland. A meta-analysis of 21,178 patients undergoing open or endovascular repair of abdominal aortic aneurysm. Br. J. Surg., 95(6):677-684, 2008.

J. Lu, X. Zhou, and M. L. Raghavan. Inverse elastostatic stress analysis in predeformed biological structures: Demonstration using abdominal aortic aneurysms. J. Biomech., 40(3):693-696, 2007. 
J. Lu, X. Zhou, and M. L. Raghavan. Inverse method of stress analysis for cerebral aneurysms. Biomech. Model. Mechanobiol., 7(8):477-486, 2008.

E. Magid, O. Soldea, and E. Rivlin. A comparison of Gaussian and mean curvature estimation methods on triangular meshes of range image data. Computer Vision and Image Understanding, 107(3):139-159, 2007.

M. A. Merkx, M. van 't Veer, M. Breeuwer, E. M. Bosboom, and F. N. van de Vosse. Importance of initial stress incorporation in abdominal aortic aneurysm wall motion: dynamic MRI validated finite element analysis. J. Biomech., 42(14): 2369-2373, 2009.

W. R. Mower, W. J. Quiñones, and S. S. Gambhir. Effect of intraluminal thrombus on abdominal aortic aneurysm wall stress. J. Vasc. Surg., 26(4):602-608, 1997.

M. Nakamura, R. Tachieda, H. Niinuma, A. Ohira, S. Endoh, K. Hiramori, and S. Makita. Circulating biochemical marker levels of collagen metabolism are abnormal in patients with abdominal aortic aneurysm. Angiology, 51(5):385-392, 2000 .

S. C. Nicholls, J. B. Gardner, M. H. Meissner, and H. K. Johansen. Rupture in small abdominal aortic aneurysms. J. Vasc. Surg., 28(5):884-888, 1998.

S. D. Ollabarriaga, J.-M. Rouet, M. Fradkin, M. Breeuwer, and W. J. Niessen. Segmentation of thrombus in abdominal aortic aneurysms from CTA with nonparametric statistical grey level appearance modeling. IEEE Transactions on Medical Imaging, (24):477-485, 2005.

Y. Papaharilaou, J. A. Ekaterinaris, E. Manousaki, and A. N. Katsamouris. A decoupled fluid structure approach for estimating wall stress in abdominal aortic aneurysms. J. Biomech., 40(2):367-377, 2007.

S. Pappu, A. Dardik, H. Tagare, and R. J. Gusberg. Beyond fusiform and saccular: a novel quantitative tortuosity index may help classify aneurysm shape and predict aneurysm rupture potential. Ann. Vasc. Surg., 22(1):88-97, 2008.

H. J. Pleumeekers, A. W. Hoes, E. van der Does, H. van Urk, A. Hofman, P. T. de Jong, and D. E. Grobbee. Aneurysms of the abdominal aorta in older adults. The Rotterdam study. Am. J. Epidemiol., 142(12):1291-1299, 1995.

M. L. Raghavan and D. A. Vorp. Toward a biomechanical tool to evaluate rupture potential of abdominal aortic aneurysm: identification of a finite strain constitutive model and evaluation of its applicability. J. Biomech., 33(4):475-482, 2000 .

M. L. Raghavan, D. A. Vorp, M. P. Federle, M. S. Makaroun, and M. W. Webster. Wall stress distribution on three-dimensionally reconstructed models of human abdominal aortic aneurysm. J. Vasc. Surg., 31(4):760-769, 2000. 
M. L. Raghavan, M. F. Fillinger, S. P. Marra, B. P. Naegelein, and F. E. Kennedy. Automated methodology for determination of stress distribution in human abdominal aortic aneurysm. J. Biomech. Eng., 127(5):868-871, 2005.

M. L. Raghavan, J. Kratzberg, E. M. Castro de Tolosa, M. M. Hanaoka, P. Walker, and E. S. da Silva. Regional distribution of wall thickness and failure properties of human abdominal aortic aneurysm. J. Biomech., 39(16):3010-3016, 2006a.

M. L. Raghavan, B. Ma, and M. F. Fillinger. Non-invasive determination of zeropressure geometry of arterial aneurysms. Ann. Biomed. Eng., 34(9):1414-1419, 2006b.

P. M. Ridker and J. D. Silvertown. Inflammation, C-reactive protein, and atherothrombosis. J. Periodontol., 79(8 Suppl):1544-1551, 2008.

R. B. Rutherford. Randomized EVAR trials and advent of level i evidence: a paradigm shift in management of large abdominal aortic aneurysms? Semin. Vasc. Surg., 19(2):69-74, 2006.

R. M. Sandford, M. J. Bown, R. D. Sayers, G. Fishwick, N. J. London, and A. Nasim. Endovascular abdominal aortic aneurysm repair: 5-year follow-up results. Ann. Vasc. Surg., 22(3):372-378, 2008.

J. Satta, E Läärä, and T. Juvonen. Intraluminal thrombus predicts rupture of an abdominal aortic aneurysm. J. Vasc. Surg., 23(4):737-739, 1996.

G. W. Schurink, J. M. van Baalen, M. J. Visser, and J. H. van Bockel. Thrombus within an aortic aneurysm does not reduce pressure on the aneurysmal wall. $J$. Vasc. Surg., 31(3):501-506, 2000.

M. H. Sherebrin, H. A. Bernans, and M. R. Roach. Extensibility changes of calcified soft tissue strips from human aorta. Can. J. Physiol. Pharmacol., 65(9):1878-1883, 1987.

K. Shimizu, R. N. Mitchell, and P. Libby. Inflammation and cellular immune responses in abdominal aortic aneurysms. Arterioscler. Thromb. Vasc. Biol., 26(5):987-994, 2006 .

C. L. Siegel, R. H. Cohan, M. Korobkin, M. B. Alpern, D. L. Courneya, and R. A. Leder. Abdominal aortic aneurysm morphology: CT features in patients with ruptured and nonruptured aneurysms. AJR Am. J. Roentgenol., 163(5):11231129, 1994.

L. Speelman, A. Bohra, E. M. Bosboom, G. W. Schurink, F. N. van de Vosse, M. S. Makaroun, and D. A. Vorp. Effects of wall calcifications in patient-specific wall stress analyses of abdominal aortic aneurysms. J. Biomech. Eng., 129(1):105-109, 2007. 
L. Speelman, E. M. Bosboom, G. W. Schurink, F. A. Hellenthal, J. Buth, M. Breeuwer, M. J. Jacobs, and F. N. van de Vosse. Patient-specific AAA wall stress analysis: 99-percentile versus peak stress. Eur. J. Vasc. Endovasc. Surg., 36(6):668-676, 2008.

L. Speelman, E. M. Bosboom, G. W. Schurink, J. Buth, M. Breeuwer, M. J. Jacobs, and F. N. van de Vosse. Initial stress and nonlinear material behavior in patientspecific AAA wall stress analysis. J. Biomech., 42(11):1713-1719, 2009a.

L. Speelman, G. W. Schurink, E. M. Bosboom, J. Buth, M. Breeuwer, F. N. van de Vosse, and M. J. Jacobs. The mechanical role of thrombus on the growth rate of an AAA. J. Vasc. Surg., In press, 2009b.

P. A. Stonebridge, T. Draper, J. Kelman, J. Howlett, P. L. Allan, R. Prescott, and C. V. Ruckley. Growth rate of infrarenal aortic aneurysms. Eur. J. Vasc. Endovasc. Surg., 11(1):70-73, 1996.

J. Thanos, M. Rebeira, B. W. Shragge, and D. Urbach. Vascular ultrasound screening for asymptomatic abdominal aortic aneurysm. Healthc. Policy, 4(2):75-83, 2008.

M. J. Thubrikar, J. Al Soudi, and F. Robicsek. Wall stress studies of abdominal aortic aneurysm in a clinical model. Ann. Vasc. Surg., 15(3):355-366, 2001.

M. J. Thubrikar, F. Robicsek, M. Labrosse, V. Chervenkoff, and B. L. Fowler. Effect of thrombus on abdominal aortic aneurysm wall dilation and stress. J. Cardiovasc. Surg. (Torino), 44(1):67-77, 2003.

M. Truijers, J. A. Pol, L. J. Schultzekool, S. M. van Sterkenburg, M. F. Fillinger, and J. D. Blankensteijn. Wall stress analysis in small asymptomatic, symptomatic and ruptured abdominal aortic aneurysms. Eur. J. Vasc. Endovasc. Surg., 33(4): 401-407, 2007.

M. Truijers, M. F. Fillinger, K. W. Renema, S. P. Marra, L. J. Oostveen, H. A. Kurvers, L. J. Schultzekool, and J. D. Blankensteijn. In-vivo changes in abdominal aortic aneurysm thrombus volume during the cardiac cycle. J. Endovasc. Ther., $16: 314-319,2009$

S. Urbonavicius, G. Urbonaviciene, B. Honore, E. W. Henneberg, H. Vorum, and J. S. Lindholt. Potential circulating biomarkers for abdominal aortic aneurysm expansion and rupture - a systematic review. Eur. J. Vasc. Endovasc. Surg., 36 (3):273-280, 2008.

T. Vainas, T. Lubbers, F. R. Stassen, S. B. Herngreen, M. P. Dieijen-Visser, C. A. Bruggeman, P. J. Kitslaar, and G. W. Schurink. Serum C-reactive protein level is associated with abdominal aortic aneurysm size and may be produced by aneurysmal tissue. Circulation, 107(8):1103-1105, 2003. 
E. A. van Dam, S. D. Dams, G. W. Peters, M. C. Rutten, G. W. Schurink, J. Buth, and F. N. van de Vosse. Non-linear viscoelastic behavior of abdominal aortic aneurysm thrombus. Biomech. Model. Mechanobiol., 7(2):127-137, 2008.

F. N. van de Vosse, J. de Hart, C. H. van Oijen, D. Bessems, T. W. Gunther, A. Segal, B. Wolters, J. M. Stijnen, and F. Baaijens. Finite-element-based computational methods for cardiovascular fluid-structure interaction. J. Eng. Math., 47:335-368, 2003.

J. A. van der Vliet and A. P. Boll. Abdominal aortic aneurysm. Lancet, 349(9055): 863-866, 1997.

M. van 't Veer, J. Buth, M. Merkx, P. Tonino, H. van den Bosch, N. Pijls, and F. N. van de Vosse. Biomechanical properties of abdominal aortic aneurysms assessed by simultaneously measured pressure and volume changes in humans. J. Vasc. Surg., 48:1401-1407, 2008.

J. P. vande Geest, E. S. di Martino, A. Bohra, M. S. Makaroun, and D. A. Vorp. A biomechanics-based rupture potential index for abdominal aortic aneurysm risk assessment: demonstrative application. Ann. N.Y. Acad. Sci., (1085):11-21, 2006a.

J. P. vande Geest, M. S. Sacks, and D. A. Vorp. A planar biaxial constitutive relation for the luminal layer of intra-luminal thrombus in abdominal aortic aneurysms. $J$. Biomech., 39(13):2347-2354, 2006b.

J. P. vande Geest, D. H. Wang, S. R. Wisniewski, M. S. Makaroun, and D. A. Vorp. Towards a noninvasive method for determination of patient-specific wall strength distribution in abdominal aortic aneurysms. Ann. Biomed. Eng., 34(7):1098-1106, 2006c.

M. Vega de Céniga, R. Gomez, L. Estallo, L. Rodriguez, M. Baquer, and A. Barba. Growth rate and associated factors in small abdominal aortic aneurysms. Eur. J. Vasc. Endovasc. Surg., 31(3):231-236, 2006.

M. Vega de Céniga, R. Gomez, L. Estallo, N. de la Fuente, B. Viviens, and A. Barba. Analysis of expansion patterns in $4-4.9 \mathrm{~cm}$ abdominal aortic aneurysms. Ann. Vasc. Surg., 22(1):37-44, 2008.

M. Vega de Céniga, M. Esteban, J. M. Quintana, A. Barba, L. Estallo, N. de la Fuente, B. Viviens, and J. L. Martin-Ventura. Search for serum biomarkers associated with abdominal aortic aneurysm growth - a pilot study. Eur. J. Vasc. Endovasc. Surg., $37(3): 297-299,2009$.

A. K. Venkatasubramaniam, M. J. Fagan, T. Mehta, K. J. Mylankal, B. Ray, G. Kuhan, I. C. Chetter, and P. T. McCollum. A comparative study of aortic wall stress using finite element analysis for ruptured and non-ruptured abdominal aortic aneurysms. Eur. J. Vasc. Endovasc. Surg., 28(2):168-176, 2004. 
D. A. Vorp. Fluid mechanical considerations in vascular grafts. overview. ASAIO J., 43(3):237-238, 1997.

D. A. Vorp and J. P. vande Geest. Biomechanical determinants of abdominal aortic aneurysm rupture. Arterioscler. Thromb. Vasc. Biol., 25(8):1558-1566, 2005.

D. A. Vorp, P. C. Lee, D. H. Wang, M. S. Makaroun, E. M. Nemoto, S. Ogawa, and M. W. Webster. Association of intraluminal thrombus in abdominal aortic aneurysm with local hypoxia and wall weakening. J. Vasc. Surg., 34(2):291-299, 2001.

D. H. Wang, M. S. Makaroun, M. W. Webster, and D. A. Vorp. Mechanical properties and microstructure of intraluminal thrombus from abdominal aortic aneurysm. $J$. Biomech. Eng., 123(6):536-539, 2001.

D. H. Wang, M. S. Makaroun, M. W. Webster, and D. A. Vorp. Effect of intraluminal thrombus on wall stress in patient-specific models of abdominal aortic aneurysm. J. Vasc. Surg., 36(3):598-604, 2002.

W. R. Wilson, E. C. Schwalbe, J. L. Jones, P. R. Bell, and M. M. Thompson. Matrix metalloproteinase 8 (neutrophil collagenase) in the pathogenesis of abdominal aortic aneurysm. Br. J. Surg., 92(7):828-833, 2005.

W. R. Wilson, M. Anderton, E. C. Choke, J. Dawson, I. M. Loftus, and M. M. Thompson. Elevated plasma mmp1 and mmp9 are associated with abdominal aortic aneurysm rupture. Eur. J. Vasc. Endovasc. Surg., 35(5):580-584, 2008.

Y. G. Wolf, W. S. Thomas, F. J. Brennan, W. G. Goff, M. J. Sise, and E. F. Bernstein. Computed tomography scanning findings associated with rapid expansion of abdominal aortic aneurysms. J. Vasc. Surg., 20(4):529-535, 1994.

B. J. Wolters, M. C. Rutten, G. W. Schurink, U. Kose, J. de Hart, and F. N. van de Vosse. A patient-specific computational model of fluid-structure interaction in abdominal aortic aneurysms. Med. Eng. Phys., 27(10):871-883, 2005.

B. J. Wolters, M. Emmer, M. C. Rutten, G. W. Schurink, and F. N. van de Vosse. Assessment of endoleak significance after endovascular repair of abdominal aortic aneurysms: a lumped parameter model. Med. Eng. Phys., 29(10):1106-1118, 2007. 


\section{Samenvatting}

Het risico op ruptuur van een aneurysma in de abdominale aorta (AAA) wordt voornamelijk geschat aan de hand van de maximale diameter van de verwijding en, in mindere mate, de groeisnelheid van de maximale diameter (Greenhalgh et al., 1998). Uit eerder onderzoek is echter gebleken dat de maximale diameter soms het ruptuurrisico onderschat, wat kan leiden tot een onverwachte ruptuur, terwijl het risico in andere gevallen overschat wordt (Darling et al., 1977; Lederle et al., 2002). In de laatste situatie wordt de patiënt blootgesteld aan onnodige operatierisico's.

Vanuit het oogpunt van een biomedisch ingenieur treedt een ruptuur van een AAA op wanneer lokaal de spanning in de wand, als gevolg van de bloeddruk, groter wordt dan de wandsterkte. Wandspanning zou daarnaast ook invloed kunnen hebben op de groeisnelheid van een AAA, aangezien een toename in spanning zou kunnen leiden tot meer schade in de wand. Dit zou kunnen leiden tot het remodelleren en groeien van het aneurysma. Het doel van het onderzoek in dit proefschrift is om AAA wandspanningsanalyses te optimaliseren en standaardiseren voor toekomstige diagnostische doeleinden, door het uitvoeren van patiënt-specifieke wandspanningsanalyses op verschillende niveaus van complexiteit. Tevens is de relatie tussen wandspanning, AAA groeisnelheid en biomarkerconcentraties bestudeerd.

Om wandspanningsanalyses in de toekomst in de kliniek te kunnen introduceren, heeft Philips Healthcare (Best, Nederland) software ontwikkeld in samenwerking met het Universitair Medisch Centrum Utrecht, Philips Research (Parijs, Frankrijk), de Technische Universiteit Eindhoven en onze groep (Breeuwer et al., 2008; de Putter et al., 2007; Delingette, 1994; Gérard et al., 2002; Ollabarriaga et al., 2005). Dit softwarepakket, HemoDyn, is ontwikkeld binnen het Philips Viewforum softwarepakket, wat in veel medische centra reeds gebruikt wordt voor visualisatie en analyse van medische beelden. HemoDyn is voor het grootste deel van het onderzoek in dit proefschrift gebruikt voor de automatische segmentatie van de AAA's en het maken van de patiënt-specifieke eindige elementen modellen.

Essentieel voor standaardisatie van spanningsanalyses is een reproduceerbare methode. In hoofdstuk $\mathbf{2}$ is de reproduceerbaarheid van HemoDyn bepaald, door wandspanningsanalyses uit te laten voeren op basis van CTA data van 20 AAA patiënten, door 3 verschillende gebruikers met een verschillende achtergrond. Naast de piekspanning is ook de 99-percentiel spanning berekend, zijnde de hoogste spanning in de wand, wanneer $1 \%$ van het wandoppervlak met de hoogste spanning niet wordt meegenomen. De piekspanning per AAA varieerde sterk tussen en binnen 
de verschillende gebruikers. Kleine subtiele verschillen in de vorm van het AAA, als gevolg van de verschillende segmentaties, hadden een sterk effect op de piekspanning. De 99-percentiel spanning was minder gevoelig voor deze kleine geometrische variaties en voor de expertise en ervaring van de gebruikers. De 99-percentiel spanning is dus een robuustere en reproduceerbaardere spanningsmaat dan de piekspanning. De 99percentiel spanning en de maximale AAA diameter waren sterk gecorreleerd, maar meer onderzoek is nodig om de nauwkeurigheid aan te tonen van de 99-percentiel spanning om ruptuurrisico in te schatten.

Naast de reproduceerbaarheid van de methode is het ook belangrijk om het effect van verschillende aannames in het model te evalueren. In hoofdstuk $\mathbf{3}$ is het effect bestudeerd op de wandspanning van initiële wandspanning en het niet-lineaire materiaalgedrag van de AAA wand. Tijdens de CTA scan wordt het aneurysma belast door de bloeddruk waardoor er een initiële spanning in de wand heerst. Uit de resultaten bleek dat wanneer deze initiële spanning niet meegenomen wordt in de spanningsanalyses, dit leidt tot een verandering in de spanning die verschilt voor ieder AAA. Hetzelfde bleek wanneer het niet-lineaire materiaalgedrag van de AAA wand niet wordt meegenomen. In de toekomst moeten daarom in AAA wandspanningsanalyses de initiële spanning en het niet-lineaire materiaal gedrag voor de wand worden meegenomen.

De aanwezigheid van thrombus en calcificaties in een aneurysma kunnen een sterk effect hebben op de wandspanning. In hoofdstuk $\mathbf{4}$ is het mechanische effect van thrombus op de AAA wandspanning bestudeerd, aan de hand van ideale en patiëntspecifieke AAA modellen. Daarnaast is de groeisnelheid van de AAA's vergeleken voor AAA's met relatief kleine en grote thrombi. Ondanks het feit dat een grotere thrombus een verlaging van de wandspanning tot gevolg heeft, bleken AAA's met een grotere thrombus sneller te groeien. Mogelijk wordt de sterkte van de wand beïnvloed door de aanwezigheid van de thrombus en speelt dit een grotere rol in het groeiproces van een AAA dan de wandspanning (vande Geest et al., 2006c; Vorp et al., 2001)

In hoofdstuk 5 is het effect van calcificaties in de AAA wand op de wandspanning bestudeerd. Het effect van de calcificaties werd bepaald door hun lokatie, vorm en stijfheid en kon niet worden afgeleid uit enkel de hoeveelheid kalk die aanwezig was in ieder AAA. Lokatie en vorm van de calcificaties kunnen eenvoudig uit de CTA data worden bepaald, maar verder onderzoek is nodig om de exacte materiaaleigenschappen en de interactie van de calcificaties met het omliggende weefsel te bepalen.

In hoofdstuk 6 is de eerste stap gezet naar de klinische applicatie van wandspanningsanalyses in AAA's. De wandspanning van een relatief kleine groep patiënten is vergeleken met de groeisnelheid en biomarkerconcentraties uit het bloed. De hypothese was dat met toenemende wandspanning ook de schade en degeneratie in de wand zou toenemen, wat kan leiden tot een snellere AAA groei en een verandering in de biomarkersconcentraties. Een relatief hogere wandspanning bleek inderdaad gerelateerd te zijn aan een snellere groei. Ondanks dat er een trend was in de concentratie van bepaalde biomarkers als functie van de wandspanning, werden er geen significante relaties tussen spanning en biomarkers gevonden.

Terugkijkend naar het doel van het onderzoek kan worden geconcludeerd dat de wandspanningsanalyses gedeeltelijk zijn geoptimaliseerd en gestandaardiseerd en dat 
een robuuste en reproduceerbare spanningsmaat is geïntroduceerd in de vorm van de 99-percentiel spanning. Ook is aangetoond dat de initiële wandspanning en het niet-lineaire materiaalgedrag van de AAA wand belangrijke componenten zijn in de spanningsanalyses. Ook thrombus en calcificaties hebben een specifiek effect op de wandspanning per aneurysma, echter, om het mechanische effect van beide factoren compleet te begrijpen is meer onderzoek nodig. AAA wandspanning lijkt gerelateerd te zijn aan groeisnelheid van het aneurysma, maar een significante relatie met biomarkers kan op dit moment niet worden aangetoond. Samenvattend is in dit proefschrift de basis gelegd voor de introductie van wandspanningsanalyses in de klinische praktijk, maar is toekomstig onderzoek nodig om de analyses verder te optimaliseren en om te evalueren wat de klinische relevantie is van wandspanningsanalyses voor aneurysmagroei en ruptuurvoorspelling. 
102 Samenvatting 


\section{Dankwoord}

$\mathrm{Na}$ ruim vier jaar met heel veel plezier werken in het azM is het af. Het proefschrift is gedrukt en ik ben er trots op. Ik heb dit onderzoek uiteraard niet alleen gedaan, maar met medewerking van vele anderen. Op deze plaats wil ik iedereen bedanken die mij hiermee, in wetenschappelijke en sociale zin, geholpen heeft.

Allereerst wil ik Prof. Jacobs en Frans bedanken voor de mogelijkheid om mijn promotie uit te voeren voor het azM en de TU/e. Prof. Jacobs, de klinische waarde was misschien niet altijd even duidelijk, maar ik denk dat we goede stappen hebben gezet op het gebied van de klinische toepassing van AAA spanningsanalyse. Frans, als tweede promotor ben jij altijd erg betrokken geweest bij het onderzoek en de (bijna) wekelijkse besprekingen heb ik altijd erg gewaardeerd. Ontzettend bedankt hiervoor. Geert Willem en Mariëlle, ik wil jullie bedanken voor de rol als dagelijkse begeleiders en tevens co-promotoren. Geert Willem, de klinische input in dit onderzoek kwam vooral van jouw hand. Regelmatig bleken jouw inzicht en ervaring veel waard, nadat een ingenieur weer eens iets onpraktisch bedacht had. Ontzettend bedankt en ik hoop dat ook jij snel mag 'promoveren'. Mariëlle, mijn dagelijkse steun en toeverlaat. Door onze nuttige discussies, veelal gevoerd in de trein, en jouw heldere kijk op het onderzoek bleef ik iedere keer weer gemotiveerd en vol ideeën. Ook jij mag trots zijn op het eind resultaat. Ontzettend bedankt!

Femke, ontzettend bedankt voor de leuke tijd en je belangrijke input in hoofdstuk 6 . Ondanks dat er een lange weg te gaan is, hebben we een goede stap gezet die wellicht tot hele nieuwe inzichten lijdt.

Ik wil ook de mensen van de afdeling BMT-azM bedanken. Ik heb een erg gezellige tijd gehad in Maastricht, waar ik nog lang met veel plezier aan terug zal denken. Wellicht dat we mekaar in de toekomst nog treffen, het blijft ten slotte een klein wereldje.

Ik wil de MRI en CT laboranten van het azM van harte bedanken voor het scannen van mijn patiënten. Het specifieke MRI protocol vergde wat aanpassingen, maar het eindresultaat was erg bevredigend. Ook de betrokkenen van het Hemodyn-project wil ik op deze plaats bedanken. Een multi-center studie is nog niet zo gemakkelijk uit te voeren, maar uiteindelijke hebben we zo een hele waardevolle database verkregen, waar in de toekomst nog veel mee gedaan kan worden.

Mijn oud-studiegenoten (en squashmaatjes) kan ik natuurlijk niet vergeten. Marcel, Inge, Alina en Homme-Auke, vele discussies zijn gevoerd en frustraties weggewerkt op en naast de squashbaan. Laten we dit in de toekomst, op welke manier dan ook, 
voortzetten. In ieder geval bedankt voor de afgelopen jaren!

Een speciale "thanks" gaat uit naar mijn paranimfen, Alina en Marcel. Enorm bedankt voor de recente steun, maar nog meer voor de steun van de afgelopen jaren. Annaly, je weet dat je een hele belangrijke rol hebt in mijn leven, maar ik wil dat hier nog even benadrukken. Ik ben erg gelukkig met je geweest en ik wil je bedanken voor je geduld en begrip in de afgelopen jaren. Als laatste wil ik mijn familie bedanken. Pap, mam, Haits, het is af. Ontzettend bedankt voor de interesse in mij en mijn werk en voor de onvoorwaardelijke steun die jullie mij geven. 


\section{Curriculum Vitae}

Lambert Speelman was born in Deventer on August $1^{\text {st }} 1980$. In 1998, he graduated the secondary education (VWO) at the Alexander Hegius Lyceum, Deventer. That year, he started Biomedical Engineering at the Eindhoven University of Technology and obtained his Master's degree in Medical Engineering in 2004 as one of the first five Medical Engineers in Europe. His Master's project was done at the University Medical Center of Maastricht, including a three-month internship at the laboratory of Dr. David Vorp, at the University of Pittsburgh, USA. The subject of the project was stress analyses on abdominal aortic aneurysms, and after his Master, he continued on this subject in a $\mathrm{PhD}$ project, of which the result is now in your hands.

Lambert continued in the field of biomedical research. From June 2009, he is employed as Postdoc at the Erasmus Medical Center, Rotterdam, at the biomechanics lab. His project aims to determine biomechanical factors that influence rupture of atherosclerotic plaques in mice. 


\section{List of Publications}

\section{Articles}

Speelman L, Bohra A, Bosboom EMH, Schurink GWH, van de Vosse FN, Makaroun MS, and Vorp DA. Effects of wall calcifications in patient-specific wall stress analyses of abdominal aortic aneurysms. J. Biomech. Eng. 2007; 129(1):105-109.

Speelman L, Bosboom EMH, Schurink GWH, Hellenthal FA, Buth J, Breeuwer M, Jacobs MJ, and van de Vosse FN. Patient-specific AAA wall stress analysis: 99percentile versus peak stress. Eur. J. Vasc. Endovasc. Surg. 2008; 26(6):668-676.

Breeuwer M, de Putter S, Kose U, Speelman L, Visser K, Gerritsen F, Hoogeveen R, Krams R, van den Bosch H, Buth J, Gunther, T, Wolters BJ, van Dam EA, and van de Vosse FN. Towards patient-specific risk assessment of abdominal aortic aneurysm. Med. Biol. Eng Comput. 2008; 46(11):1085-1095.

Speelman L, Bosboom EMH, Schurink GWH, Buth J, Breeuwer M, Jacobs MJ, and van de Vosse FN. Initial stress and nonlinear material behavior in patient-specific AAA wall stress analysis. J. Biomech. 2009; 42(11):1713-1719.

Merkx MA, van 't Veer M, Speelman L, Breeuwer M, Buth J, and F. N. van de Vosse. Importance of initial stress incorporation in Abdominal Aortic Aneurysm wall motion: dynamic MRI validated Finite Element Analysis. J. Biomech. 2009; 42(14):2369-2373.

Speelman L, Schurink GWH, Bosboom EMH, Buth J, Breeuwer M, van de Vosse FN, and Jacobs MJ. The mechanical role of thrombus on the growth rate of an AAA. J. Vasc. Surg. 2009; In press.

Speelman L*, Hellenthal FA*, Pulinx B, Bosboom EMH, van de Vosse FN, Jacobs MJ, Breeuwer M, van Sambeek MJ, Wodzig WK, and Schurink GWH. The influence of wall stress on AAA growth and biomarkers. Eur. J. Vasc. Endovasc. Surg. 2009; Submitted.

* authors contributed equally 


\section{Refereed proceedings}

Speelman L, Bohra A, Makaroun MS, and Vorp DA. Assessment of wall calcification in patient-specific finite element analyses of abdominal aortic aneurysms. Summer Bioengineering Conference Proceedings 2005; Vail, Colorado, United States.

Speelman L, Wolters BJ, Emmer M, Bosboom EMH, Schurink GWH, and van de Vosse FN. Assessment of endoleak significance after endovascular repair of abdominal aortic aneurysms: a lumped parameter model. Summer Bioengineering Conference Proceedings 2006; Amelia Island, Florida, United States.

Speelman L, van Dam EA, Peters GWM, Bosboom EMH, Rutten MCM, Schurink GWH, Jacobs MJ, and van de Vosse FN. Intraluminal thrombus in AAA wall stress analysis. Summer Bioengineering Conference Proceedings 2007; Keystone, Colorado, United States.

Speelman L, Bosboom EMH, Hellenthal FAM, Schurink GWH, Jacobs MJ, and van de Vosse FN. 99 percentile wall stress and not peak stress correlates with AAA diameter. Summer Bioengineering Conference Proceedings 2008; Marco Island, Florida, United States.

Speelman L, Bosboom EMH, Schurink GWH, Buth J, Breeuwer M, Jacobs MJ, and van de Vosse FN. Initial stress and nonlinear material behavior in patient-specific AAA wall stress analysis. Summer Bioengineering Conference proceedings 2009; Lake Tahoe, California, United States. 